\title{
Search for doubly charged scalar bosons decaying into same-sign $W$ boson pairs with the ATLAS detector
}

\author{
ATLAS Collaboration ${ }^{\star}$ \\ CERN, 1211 Geneva 23, Switzerland
}

Received: 7 August 2018 / Accepted: 7 December 2018 / Published online: 23 January 2019

(C) CERN for the benefit of the ATLAS collaboration 2019

\begin{abstract}
A search for doubly charged scalar bosons decaying into $W$ boson pairs is presented. It uses a data sample from proton-proton collisions corresponding to an integrated luminosity of $36.1 \mathrm{fb}^{-1}$ collected by the ATLAS detector at the LHC at a centre-of-mass energy of $13 \mathrm{TeV}$ in 2015 and 2016. This search is guided by a model that includes an extension of the Higgs sector through a scalar triplet, leading to a rich phenomenology that includes doubly charged scalar bosons $H^{ \pm \pm}$. Those bosons are produced in pairs in protonproton collisions and decay predominantly into electroweak gauge bosons $H^{ \pm \pm} \rightarrow W^{ \pm} W^{ \pm}$. Experimental signatures with several leptons, missing transverse energy and jets are explored. No significant deviations from the Standard Model predictions are found. The parameter space of the benchmark model is excluded at $95 \%$ confidence level for $H^{ \pm \pm}$bosons with masses between 200 and $220 \mathrm{GeV}$.
\end{abstract}

\section{Introduction}

An extension of the scalar sector of the Standard Model (SM) is possible in the context of type II seesaw models [1], originally conceived to explain the smallness of the neutrino masses. In the model investigated in this paper, the scalar sector includes a hypercharge $Y=2$ scalar triplet, $\Delta$, in addition to the SM scalar doublet $H[2,3]$. Electroweak symmetry breaking (EWSB) is achieved if the neutral components of $H$ and $\Delta$ acquire vacuum expectation values, $v_{d}$ and $v_{t}$ respectively. After the EWSB, the mixing between these fields results in seven scalar bosons: $H^{ \pm \pm}, H^{ \pm}, A^{0}$ (CP odd), $H^{0}$ (CP even), $h^{0}$ (CP even). A small mixing between the CPeven scalars allows $h^{0}$ to have the expected properties of the SM Higgs boson. In addition, the triplet-neutrino Yukawa term provides non-zero neutrino masses proportional to the vacuum expectation value of the triplet $v_{t}$. Constraints from electroweak precision measurements lead to an upper bound on $v_{t}$ of around $1 \mathrm{GeV}$. This range is significantly lower than the electroweak scale and matches the need for small values

^e-mail: atlas.publications@ @ern.ch suggested by the natural association of $v_{t}$ with the neutrino masses.

The assumption of a non-zero $v_{t}$, of the order of a hundred $\mathrm{MeV}$, opens the possibility for the doubly charged boson to decay into a pair of same-sign $W$ bosons, $H^{ \pm \pm} \rightarrow W^{ \pm} W^{ \pm}$, while the leptonic decays $H^{ \pm \pm} \rightarrow \ell^{ \pm} \ell^{ \pm}$are suppressed with increasing $v_{t}[4,5]$. Extensive searches for leptonic decays $H^{ \pm \pm} \rightarrow \ell^{ \pm} \ell^{ \pm}$have been performed at various colliders [6-11], where $H^{ \pm \pm}$bosons with masses up to about $800 \mathrm{GeV}$ have been excluded. Moreover, searches for $H^{ \pm \pm} \rightarrow W^{ \pm} W^{ \pm}$decays have been performed by the CMS Collaboration in the context of single $H^{ \pm \pm}$production through vector-boson fusion at large $v_{t}$ (of order of tens of $\mathrm{GeV}$ ) [12,13] for a model with two Higgs triplets [14]. For that model, a custodial symmetry avoids large contributions to the electroweak precision observables [15]. In contrast, the $H^{ \pm \pm} \rightarrow W^{ \pm} W^{ \pm}$decay mode has not been directly searched for so far for small values of $v_{t}$, where the vector-boson fusion is suppressed.

The present paper focuses on the phenomenology of doubly charged scalar bosons $H^{ \pm \pm}$that can be produced in pairs at the Large Hadron Collider (LHC) and decay into $W$ bosons. The triplet vacuum expectation value is taken to be $v_{t}=0.1 \mathrm{GeV}$ such that only the $H^{ \pm \pm} \rightarrow W^{ \pm} W^{ \pm}$decays are relevant, leading to final states with four $W$ bosons. The mixing between the CP-even scalars is taken to be $10^{-4}$ and the remaining five Yukawa parameters in the potential are adjusted to obtain a given $H^{ \pm \pm}$mass hypothesis while requiring $h^{0}$ to have a mass of $125 \mathrm{GeV}$. The corresponding crosssection calculation is performed for on-shell $W$ bosons, and therefore only the region $m_{H^{ \pm \pm}}>200 \mathrm{GeV}$ is considered in the present analysis.

The four-boson final states are identified by the presence of light charged leptons (electrons or muons), missing transverse momentum, and jets. The analysis uses three final states defined according to the number of light leptons: same-sign (SS) dilepton channel $\left(2 \ell^{\mathrm{ss}}\right)$, trilepton channel ( $\left.3 \ell\right)$ and fourlepton channel $(4 \ell)$. Similar final states were used for other searches for new phenomena in ATLAS [16-18]. However, 
the previously searched signal topologies differ significantly from those targeted in the present analysis and a dedicated event selection optimisation is therefore applied.

This paper includes a description of the experimental setup in Sect. 2, followed by a description of the simulation used in the analysis in Sect. 3. The event selection and background estimations for the three explored signatures are described in Sect. 4. The signal region optimisation is described in Sect. 5. The systematic uncertainties are presented in Sect. 6. The results are shown in Sect. 7, followed by the conclusions in Sect. 8 .

\section{ATLAS detector}

The ATLAS experiment [19] at the LHC is a multipurpose particle detector with a forward-backward symmetric cylindrical geometry and a near $4 \pi$ coverage in solid angle. ${ }^{1}$ It consists of an inner tracking detector surrounded by a superconducting solenoid providing a $2 \mathrm{~T}$ axial magnetic field, electromagnetic and hadronic calorimeters, and a muon spectrometer. The inner tracking detector, covering the pseudorapidity range $|\eta|<2.5$, consists of silicon pixel and silicon microstrip tracking detectors inside a transitionradiation tracker that covers $|\eta|<2$.0. It includes, for the $\sqrt{s}=13$ TeVrunning period, a newly installed innermost pixel layer, the insertable $B$-layer [20]. Lead/liquid-argon (LAr) sampling calorimeters provide electromagnetic (EM) energy measurements for $|\eta|<2.5$ with high granularity and longitudinal segmentation. A hadronic calorimeter consisting of steel and scintillator tiles covers the central pseudorapidity range $(|\eta|<1.7)$. The endcap and forward regions are instrumented with LAr calorimeters for EM and hadronic energy measurements up to $|\eta|=4$.9. The muon spectrometer surrounds the calorimeters and is based on three large aircore toroid superconducting magnets with eight coils each. It includes a system of precision tracking chambers $(|\eta|<2.7)$ and fast detectors for triggering $(|\eta|<2.4)$. A two-level trigger system is used to select events [21]. The first-level trigger is implemented in hardware and uses a subset of the detector information to reduce the accepted rate to a design maximum of $100 \mathrm{kHz}$. This is followed by a software-based trigger with a sustained average accepted event rate of about $1 \mathrm{kHz}$.

\footnotetext{
${ }^{1}$ ATLAS uses a right-handed coordinate system with its origin at the nominal interaction point (IP) in the centre of the detector and the $z$ axis along the beam pipe. The $x$-axis points from the IP to the centre of the LHC ring, and the $y$-axis points upwards. Cylindrical coordinates $(r, \phi)$ are used in the transverse plane, $\phi$ being the azimuthal angle around the $z$-axis. The pseudorapidity is defined in terms of the polar angle $\theta$ as $\eta=-\ln \tan (\theta / 2)$. Angular distance is measured in units of $\Delta R \equiv \sqrt{(\Delta \eta)^{2}+(\Delta \phi)^{2}}$.
}

\section{Data and simulation}

The data sample collected by the ATLAS Collaboration at $\sqrt{s}=13 \mathrm{TeV}$ during 2015 and 2016 was used. After the application of beam and data quality requirements, the integrated luminosity is $36.1 \mathrm{fb}^{-1}$.

Monte Carlo (MC) simulation samples were produced for signal and background processes using the full ATLAS detector simulation [22] based on GEANT4 [23] or, for selected smaller backgrounds and some of the signal samples, a fast simulation using a parameterisation of the calorimeter response and GEANT4 for the tracking system [24]. To simulate the effects of additional $p p$ collisions in the same and nearby bunch crossings (pile-up), additional interactions were generated using PYTHIA $8.186[25,26]$ with a set of tuned parameters for the underlying event, referred to as the A2 tune [27], and the MSTW2008LO set of parton distribution functions (PDF) [28], and overlaid on the simulated hard-scatter event. The simulated events were reweighted to match the distribution of the number of interactions per bunch crossing observed in the data and were reconstructed using the same procedure as for the data.

The signal events containing $H^{ \pm \pm}$pairs were simulated with the CalcHEP generator version 3.4 [29], which is at leading order in $\mathrm{QCD}$, using the Lagrangian described in Ref. [3] and the PDF set CTEQ6L1 [30,31]. The modelling of the parton showering and hadronisation of these events was performed using PYTHIA $8.186[25,26]$ with the A14 tune [32]. Event samples for the process $p p \rightarrow$ $H^{ \pm \pm} H^{\mp \mp} \rightarrow W^{ \pm} W^{ \pm} W^{\mp} W^{\mp}$ were simulated for $m_{H^{ \pm \pm}}$in the range from 200 to $700 \mathrm{GeV}$ with steps of $100 \mathrm{GeV}$. The production cross-section decreases rapidly with $m_{H^{ \pm \pm}}$and is $80.7 \mathrm{fb}$ for $m_{H^{ \pm \pm}}=200 \mathrm{GeV}, 5.0 \mathrm{fb}$ for $m_{H^{ \pm \pm}}=400 \mathrm{GeV}$, and $0.35 \mathrm{fb}$ for $m_{H^{ \pm \pm}}=700 \mathrm{GeV}$. Next-to-leading order (NLO) corrections [33] in QCD were applied, which increase these cross-sections by a factor 1.25 . The fast detector simulation was used for the samples corresponding to $m_{H^{ \pm \pm}}>$ $500 \mathrm{GeV}$.

The SM background processes were simulated using the $\mathrm{MC}$ event generator programs and configurations shown in Table 1. The production of $V V, V V q q$, and $V V V$ (where $V$ denotes a vector boson $W$ or $Z$ and $q q$ labels the vector-boson fusion production mechanism) was simulated with a NLO QCD matrix element computed by SHERPA and matched to the SHERPA parton shower. The main background contribution in the $2 \ell^{\mathrm{ss}}$ and $3 \ell$ channels is from $W Z$ production, for which the total cross-section prediction is $48.2 \pm 1.1 \mathrm{pb}$ [44]. The main contribution to the $4 \ell$ topology is from $Z Z$ production with a total cross-section of $16.9 \pm 0.6 \mathrm{pb}[45,46]$, which is suppressed by requiring significant missing transverse momentum in these events. The MC samples used to simulate $t \bar{t} H, t \bar{t} V, V V$ and $t \bar{t}$ are described in more detail in Refs. [47-49]. 


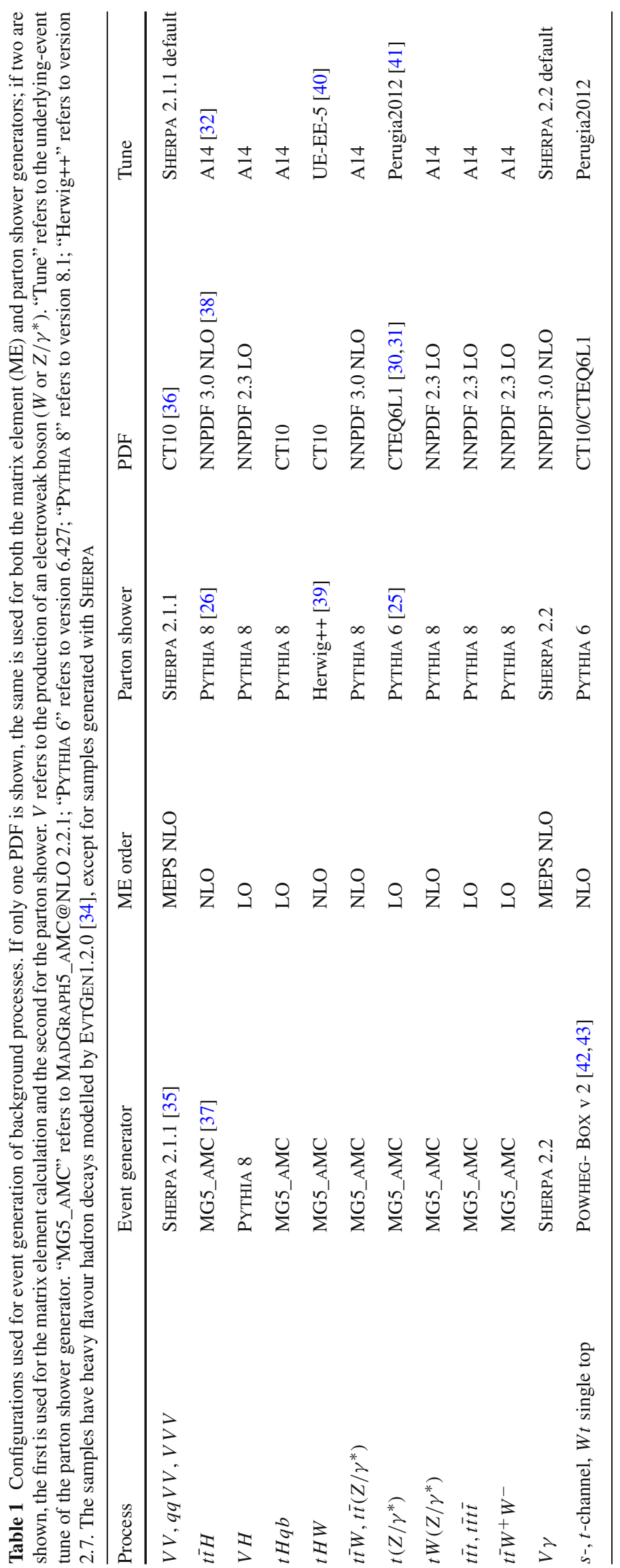


The simulated SM contributions in each of the channels considered are separated into prompt-lepton and fake-lepton contributions, depending on the source of the reconstructed leptons at generator level. The processes that contain only reconstructed charged leptons originating from prompt leptonic decays of $W$ and $Z$ bosons are classified as a promptlepton contribution, while processes with at least one of the reconstructed leptons being a misidentified hadron or photon, or a lepton from hadron decays constitute the fake-lepton contribution. The simulated events are not used to evaluate the background originating from charge-misidentified leptons for the $2 \ell^{\mathrm{ss}}$ channel and fake leptons for the $2 \ell^{\mathrm{ss}}$ and $3 \ell$ channels. These are estimated in general using data-driven methods because they are not well modelled by simulations. This is the case in particular for $Z \rightarrow \ell^{+} \ell^{-}, W \rightarrow \ell v$ and $t \bar{t}$ processes. The background process $V \gamma$ can contribute if electrons originating from the photon conversion are selected. This contribution is found to be small and adequately modelled, so it is estimated using the MC simulation. For the $4 \ell$ channel, the background from fake leptons is small and the data-driven methods are not applicable due to the low number of events available, so the MC simulation is used to estimate both the prompt-lepton and the fake-lepton contributions.

\section{Event selection and background estimates}

\subsection{Event reconstruction}

Interaction vertices originating from $p p$ collisions are reconstructed using at least two tracks with transverse momentum $p_{\mathrm{T}}>0.4 \mathrm{GeV}$, and required to be consistent with the beamspot envelope. The primary vertex is identified as the vertex with the largest sum of squares of the transverse momenta from associated tracks [50].

Electrons are reconstructed as tracks in the inner detector matched to clusters in the electromagnetic calorimeter, within the region of pseudorapidity $|\eta|<2.47$ [51]. The candidates in the transition region between the barrel and the endcap calorimeters $(1.37<|\eta|<1.52)$ are removed. Only those electron candidates with transverse momentum greater than $10 \mathrm{GeV}$ are considered. The electron identification is based on a multivariate likelihood-based discriminant that uses the shower shapes in the electromagnetic calorimeter and the associated track properties measured in the inner detector. In particular, the loose and tight identification working points, described in Ref. [51], are used, providing electron identification efficiencies of approximately $95 \%$ and $78-$ $90 \%$ (depending on $p_{\mathrm{T}}$ and $\eta$ ), respectively. In order to reduce contributions from converted photons and hadron decays, the longitudinal impact parameter of the electron track relative to the selected event primary vertex, multiplied by the sine of the polar angle, $\left|z_{0} \sin \theta\right|$, is required to be less than $0.5 \mathrm{~mm}$. The transverse impact parameter divided by its uncertainty, $\left|d_{0}\right| / \sigma\left(d_{0}\right)$, is required to be less than five. The identification algorithm is complemented by an isolation requirement, based on the energy in a cone around the electron candidate calculated using either charged tracks or calorimetric deposits. The calorimeter- and track-based isolation criteria are applied jointly to suppress fake electrons.

Muon candidates are reconstructed by combining tracks formed in the inner detector and in the muon spectrometer, within the region of pseudorapidity $|\eta|<2.5$ [52]. Only those muon candidates with transverse momentum greater than $10 \mathrm{GeV}$ are considered. A muon candidate is required to satisfy loose or tight identification criteria which are defined in Ref. [52], and which have efficiencies of approximately $98 \%$ and $92 \%$, respectively. Similarly to electrons, isolation criteria complement the identification requirements. The impact parameters must satisfy $\left|z_{0} \sin \theta\right|<0.5 \mathrm{~mm}$ and $\left|d_{0}\right| / \sigma\left(d_{0}\right)<3$ when selecting muons.

Combining the selection criteria mentioned above, two types of lepton requirements are used for both the electrons and muons: type $T$ (for tight) and $L$ (for loose). The type $T$ leptons are a subset of the type $L$.

Jets are reconstructed from topological clusters [53] of energy deposits in the calorimeters using the anti- $k_{t}$ algorithm [54,55] with a radius parameter of $R=0.4$. Only jets with $p_{\mathrm{T}}>25 \mathrm{GeV}$ and $|\eta|<2.5$ are considered. In order to suppress jets arising from pile-up collisions, jets with $p_{\mathrm{T}}<60 \mathrm{GeV}$ and $|\eta|<2.4$ must have a sizeable fraction of their tracks matched to the selected primary vertex [56]. Jets containing $b$-hadrons are identified ( $b$-tagged) via a multi-variate discriminant combining information from the impact parameters of displaced tracks with topological properties of secondary and tertiary decay vertices reconstructed within the jet [57]. The $b$-tagging algorithm used for this search has an average efficiency of $70 \%$ to identify $b$-jets with $p_{\mathrm{T}}>20 \mathrm{GeV}$ and $|\eta|<2.5$ in simulated $t \bar{t}$ events.

To avoid object double counting, an overlap removal procedure is applied to resolve ambiguities among electrons, muons, and jets in the final state. Any electron candidate sharing an inner detector track with a muon candidate is removed. Jets within $\Delta R=0.2$ of an electron, as well as jets with less than three tracks within $\Delta R=0.2$ of a muon candidate are discarded. Any remaining electron candidate within $\Delta R=0.4$ of a jet is discarded. Any remaining muon candidate within $\Delta R=0.04+10 / p_{\mathrm{T}}^{\mu}(\mathrm{GeV})$ of a jet is discarded.

The missing transverse momentum, with magnitude $E_{\mathrm{T}}^{\text {miss }}$, is defined as the negative vector sum of the transverse momenta of all identified leptons and jets and the remaining unclustered energy of the event, which is estimated from tracks associated with the primary vertex but not assigned to any physics object [58]. 


\subsection{Event preselection}

Candidate events are selected using triggers that require at least one electron or one muon to pass various thresholds of $p_{\mathrm{T}}$ [21]. The higher thresholds are applied with looser lepton identification and/or isolation requirements in order to ensure efficiencies close to $100 \%$ for leptons with transverse momentum above $30 \mathrm{GeV}$.

The signal topologies studied in this search involve the presence of at least two leptons of the same charge and are classified as explained above in three mutually exclusive categories: $2 \ell^{\mathrm{ss}}, 3 \ell$ and $4 \ell$ channels. The $2 \ell^{\mathrm{ss}}$ channel targets signal events where the two same-sign $W$ bosons from one of the doubly charged Higgs boson decays leptonically, while the two $W$ bosons from the other doubly charged Higgs boson decay hadronically. In the $3 \ell$ channel, one $W$ boson decays hadronically and in the $4 \ell$ channel, all $W$ bosons decay leptonically. All channels present significant $E_{\mathrm{T}}^{\text {miss }}$ corresponding to the neutrinos from leptonic $W$ boson decays. In the $2 \ell^{\text {ss }}$ and $4 \ell$ channels, jets from $W$ boson decays originate from the first- and second-generation quarks, and therefore lead to events without $b$-jets. The event selection is divided into two steps: the preselection and the signal region selection.

The preselection requirements are summarised in Table 2 . The electrons (muons) are selected in the pseudorapidity range $|\eta|<2.47$ (2.5) with a transverse momentum of at least $10 \mathrm{GeV}$, satisfying the type $L$ requirement. Events are selected only if the absolute value of the sum of charges of the leptons is two, one and zero for the $2 \ell^{s s}, 3 \ell$ and $4 \ell$ channels, respectively. At least one of the leptons is required to have $p_{\mathrm{T}}>30 \mathrm{GeV}$ to ensure a high trigger efficiency. To reduce the fake-lepton contamination in the $2 \ell^{\text {ss }}$ channel, the second highest $p_{\mathrm{T}}$ (subleading) lepton is required to have $p_{\mathrm{T}}$ $>20 \mathrm{GeV}$ and both leptons are required to be of type $T$.
Similarly in the $3 \ell$ channel, each lepton in the pair of leptons of the same sign, which is expected to suffer more from fakelepton contamination, is required to have $p_{\mathrm{T}}>20 \mathrm{GeV}$ and to both be of type $T$. In the $2 \ell^{\text {ss }}$ and $4 \ell$ channels, the leptons are labelled by descending $p_{\mathrm{T}}$, and are denoted by $\ell_{1,2, \ldots}$. The ranking follows a different logic for the $3 \ell$ channel: the lepton that has a charge opposite to the total lepton charge is denoted as $\ell_{0}$, while the same-sign leptons are denoted by $\ell_{1}$ and $\ell_{2}$, ranked by increasing distance to $\ell_{0}$ in the $\eta-\phi$ plane.

Further preselection requirements are based on $E_{\mathrm{T}}^{\text {miss }}$, the jet multiplicity $N_{\text {jets }}$ and the number of jets tagged as $b$-jets $N_{b \text {-jet }}$. Moreover, in order to reduce the background from $Z$ bosons and neutral mesons decaying into same-flavour opposite-sign leptons (SFOS), the invariant mass of such lepton pairs is required to be greater than $12(15) \mathrm{GeV}$ for the $3 \ell$ $(4 \ell)$ channel and to have an invariant mass that is not compatible with the $Z$ boson. For the $2 \ell^{\text {ss }}$ channel, the $Z$ boson invariant mass veto is also applied to $e^{ \pm} e^{ \pm}$events, in order to reduce the contributions originating from electron charge misidentification.

After this preselection, 562 data events are selected in the $2 \ell^{\text {ss }}$ channel, 392 events in the $3 \ell$ channel, and 44 events in the $4 \ell$ channel.

\subsection{Background estimate}

The background processes containing only prompt selected leptons are estimated with $\mathrm{MC}$ simulations normalised to the most precise cross-section calculation (see Sect. 3). Further contributions originate from non-prompt and mismeasured leptons. The procedures used to estimate those contributions are described in the following.
Table 2 The preselection criteria for the three analysis channels. The leptons are ordered by decreasing $p_{\mathrm{T}}\left(\ell_{1}, \ell_{2}, \ldots\right)$ in the $2 \ell^{\mathrm{ss}}$ and $4 \ell$ channels, while for the $3 \ell$ channel $\ell_{1}, \ell_{2}$ denote the same-sign leptons and $\ell_{0}$ the lepton with a charge opposite to the total lepton charge. $Q_{\ell}$ denotes the charge of each lepton

\begin{tabular}{|c|c|c|c|}
\hline Selection criteria & $2 \ell^{\mathrm{ss}}$ & $3 \ell$ & $4 \ell$ \\
\hline Trigger & \multicolumn{3}{|c|}{$\begin{array}{l}\text { At least one lepton with } p_{\mathrm{T}}^{\ell}>30 \mathrm{GeV} \text { that fulfils the requirements of single-lepton } \\
\text { triggers }\end{array}$} \\
\hline$N_{\ell}\left(L\right.$-type, $\left.p_{\mathrm{T}}>10 \mathrm{GeV},\left|\eta_{\ell}\right|<2.47\right)$ & 2 & 3 & 4 \\
\hline$N_{\ell}\left(T\right.$-type, $\left.p_{\mathrm{T}}>10 \mathrm{GeV},\left|\eta_{\ell}\right|<2.47\right)$ & 2 & $2\left(\ell_{1,2}\right)$ & - \\
\hline$\left|\sum Q_{\ell}\right|$ & 2 & 1 & 0 \\
\hline Lepton $p_{\mathrm{T}}$ threshold & $p_{\mathrm{T}}^{\ell_{1}, \ell_{2}}>30,20 \mathrm{GeV}$ & $p_{\mathrm{T}}^{\ell_{0}, \ell_{1}, \ell_{2}}>10,20,20 \mathrm{GeV}$ & $p_{\mathrm{T}}^{\ell_{1}, \ell_{2}, \ell_{3}, \ell_{4}}>10 \mathrm{GeV}$ \\
\hline$E_{\mathrm{T}}^{\mathrm{miss}}$ & $>70 \mathrm{GeV}$ & $>30 \mathrm{GeV}$ & $>30 \mathrm{GeV}$ \\
\hline$N_{\text {jets }}$ & $\geq 3$ & $\geq 2$ & - \\
\hline$b$-jet veto & $N_{b \text {-jet }}=0$ & $N_{b \text {-jet }}=0$ & $N_{b \text {-jet }}=0$ \\
\hline Low SFOS $m_{\ell \ell}$ veto & - & $m_{\ell^{ \pm} \ell^{\mp}}>15 \mathrm{GeV}$ & $m_{\ell^{ \pm} \ell^{\mp}}>12 \mathrm{GeV}$ \\
\hline$Z$ boson decays veto & $\left|m_{e^{ \pm} e^{ \pm}}-m_{Z}\right|>10 \mathrm{GeV}$ & $\mid m_{\ell^{ \pm} \ell^{\mp}}$ & $m_{Z} \mid>10 \mathrm{GeV}$ \\
\hline
\end{tabular}




\subsubsection{Charge misidentification}

In the $2 \ell^{\text {ss }}$ channel, a background contribution is expected from events with opposite-sign lepton pairs when the charge of one of the leptons is misidentified, while the background contribution from charge misidentification is negligible for $3 \ell$ and $4 \ell$ channels. In the transverse momentum domain relevant for this analysis, charge misidentification is only significant for electrons and is due mainly to bremsstrahlung interactions with the inner detector material. The radiated photon produces an $e^{+} e^{-}$pair near the original electron trajectory leading to a charge identification confusion.

The misidentification rate is measured using a large data sample of dilepton events originating mainly from $Z \rightarrow$ $e^{+} e^{-}$decays selected by two type $T$ electrons with an invariant mass between 80 and $100 \mathrm{GeV}$. The sample contains mostly opposite-sign dileptons, with a small fraction of samesign dileptons. The fraction of same-sign dilepton events is used to extract the charge-misidentification rate as a function of electron $p_{\mathrm{T}}$ and $\eta$. This rate is found to range between $0.02 \%$ and $10 \%$, where large values are obtained at large rapidities where the amount of material is higher. The statistical error of this estimate is taken as systematic uncertainty of the charge misidentification rate. The background from fake leptons in both the opposite-sign and same-sign samples is estimated using sidebands around the $Z$ boson mass peak. Its impact on the charge misidentification rate is about $2 \%$ and is included in the systematic uncertainty.

The background from charge misidentification in a given region is estimated using a data control sample selected with the same criteria as the nominal sample but with oppositesign dilepton pairs, where at least one lepton is an electron, weighted by the probability that the charge of the electron(s) is misidentified.

Table 3 The selection criteria defining the fake-enriched control regions used to determine the fake factors for the $2 \ell^{\text {ss }}$ and $3 \ell$ channel and the MC scale factors for the $4 \ell$ channel. The symbol "-_" means no requirement. The transverse mass $m_{\mathrm{T}}$, used for the $4 \ell-\mathrm{Z}$ region to

\subsubsection{Fake-lepton contributions}

The composition of the fake-lepton background varies considerably among the analysis channels. Therefore, the methods to estimate the fake-lepton contributions are different for the $2 \ell^{\text {ss }}, 3 \ell$ and $4 \ell$ channels. The contribution from fake leptons for the $2 \ell^{\mathrm{ss}}$ and $3 \ell$ channels are estimated using the fakefactor method, while the simulation prediction corrected with data-driven scale factors is used for the $4 \ell$ channels. Those methods involve various fake-enriched control samples that are summarised in Table 3 and described below.

Fake-lepton contribution estimate for the $2 \ell^{\text {ss }}$ channel The fake-factor method assumes that the fake-lepton contribution in a nominal region, which can be the preselection or the signal region, can be computed using an extrapolation factor that is referred to as a fake factor, and is denoted as $\theta$ in the following. The fake factor is multiplied by the number of events containing fake leptons in a region with the same selection criteria as the nominal region, except that at least one of the leptons is required to satisfy the type $L$ but not the type $T$ identification criteria. That lepton is denoted by $\phi, \mu$ or collectively $\ell$ in the following.

The fake factors are calculated in fake-enriched control regions with kinematic selections designed to enhance their content in fake leptons. In the case of the $2 \ell^{\mathrm{ss}}$ channel $\left(2 \ell^{\mathrm{ss}}\right.$ column in Table 3), this is achieved by requiring low $E_{\mathrm{T}}^{\text {miss }}$. The fake factor is defined as the number of fake-lepton events in the fake-enriched region where all selected leptons pass the type $T$ identification, divided by the number of fakelepton events in the same region but where one of the selected leptons is of type $\ell$.

The muon fake factor is thus computed in the fakeenriched region, where a pair of same-sign muons was selected, as follows:

reduce the $W Z$ contributions, is calculated as the invariant mass of the vector sum of transverse momentum of the fake-lepton candidate and the missing transverse momentum

\begin{tabular}{lllll}
\hline Sample & $2 \ell^{\text {ss }}$ & $3 \ell$ & $4 \ell-\mathrm{Z}$ & $4 \ell$-T \\
\hline$N_{\ell}$ (type $\left.L\right)$ & 2 & 3 & 3 & 3 \\
$\left|\sum Q_{\ell}\right|$ & 2 & 1 & 1 & 1 \\
$p_{\mathrm{T}}^{\ell}$ & $>30,20 \mathrm{GeV}$ & $>10,20,20 \mathrm{GeV}$ & $>10,10,10 \mathrm{GeV}$ & $>10,10,10 \mathrm{GeV}$ \\
$N_{\text {jets }}$ & $\geq 3$ & 1 & 1 or 2 & 1 or 2 \\
$N_{b \text {-jet }}$ & 0 & - & - & - \\
$p_{\mathrm{T}}^{\text {jet }}$ & $>25 \mathrm{GeV}$ & $>25 \mathrm{GeV}$ & $>25 \mathrm{GeV}$ & $>30(25) \mathrm{GeV}$ \\
$Z$-window & $\left|m_{\ell e}^{\text {ss }}-m_{Z}\right|>10 \mathrm{GeV}$ & $\left|m_{\ell \ell}^{\text {os }}-m_{Z}\right|>10 \mathrm{GeV}$ & $\left|m_{\ell \ell}^{\text {os }}-m_{Z}\right|<10 \mathrm{GeV}$ & No same-flavour \\
& - & $>15 \mathrm{GeV}$ & & opposite-sign lepton pair \\
$m_{\ell \ell}^{\text {os }}$ & - & - & - & - \\
$E_{\mathrm{T}}^{\text {miss }}$ & $<70 \mathrm{GeV}$ & - & $<50 \mathrm{GeV}$ & - \\
$m_{\mathrm{T}}$ & - & $<50 \mathrm{GeV}$ & - \\
\hline
\end{tabular}



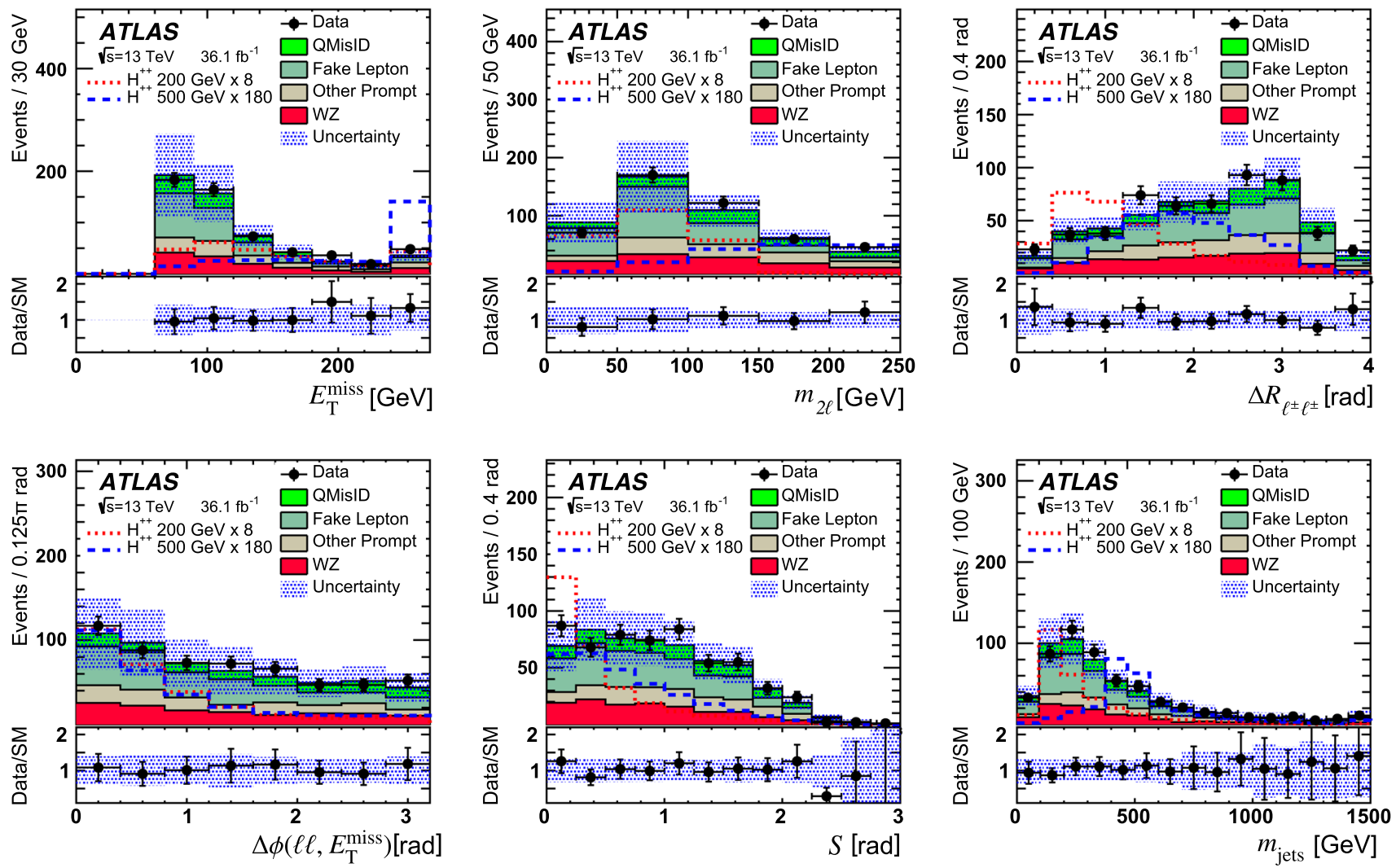

Fig. 1 Distribution of variables used for the signal region optimisation of the $2 \ell^{\mathrm{ss}}$ final state. The events are selected with the preselection requirements listed in Table 2. The data (dots) are compared with the predictions (histograms) that include the contributions from the dominant prompt-lepton background $(W Z)$, other prompt-lepton backgrounds, processes where a fake lepton is reconstructed, and electrons

$\theta_{\mu}^{2 \ell^{\text {ss }}}=\frac{\left(N^{\text {Data }}-N^{\text {Prompt }}\right)_{\mu \mu}^{C}}{\left(N^{\text {Data }}-N^{\text {Prompt }}\right)_{\mu \mu}^{C}}$,

where $N_{\mu \mu}^{\text {Data,C }}$ and $N_{\mu \mu}^{\text {Data,C }}$ are the number of events where both muons are of type $T$, and where one is of type $T$ and the other of type $\not$, respectively. The prompt-lepton contributions $N^{\text {Prompt }}$, which are estimated using MC simulation, are subtracted from data event yields to obtain a pure estimate of the fake-lepton contributions in the $\mu \mu$ and $\mu \mu$ regions. The superscript $C$ indicates the fake-enriched control region.

The electron fake factor is computed using the fakeenriched region where a same-sign $e \mu$ pair was selected:

$\theta_{e}^{2 \ell^{\text {ss }}}=\frac{\left(N^{\text {Data }}-N^{\text {Prompt }}-N^{\varnothing}-N^{\text {fake- } \mu}\right)_{e \mu}^{C}}{\left(N^{\text {Data }}-N^{\varnothing}-N^{\text {Prompt }}\right)_{\phi \mu}^{C}}$.

In addition to the prompt-lepton contribution, the electron

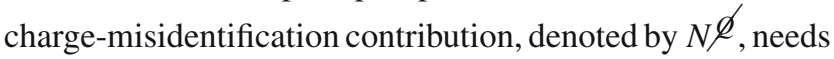
to be subtracted. It is computed using the method described in Sect. 4.3.1. Furthermore, the fake-muon contribution in the with misidentified charge (QMisID). The expected signal distributions corresponding to two $H^{ \pm \pm}$masses are also shown, scaled up for visibility. The last bin includes overflows. In each figure the bottom panel shows the ratio of data to the prediction, where the band around unity represents the total uncertainty of the SM prediction

$e \mu$ sample is subtracted from the numerator. It is computed as:

$N_{e \mu}^{\text {fake- } \mu, C}=\theta_{\mu}^{2 \ell^{\text {ss }}}\left(N^{\text {Data }}-N^{\text {Prompt }}\right)_{e \mu}^{C}$.

The fake-muon contribution is not considered in the denominator of the electron fake factor, in Eq. (2), because it is negligible.

The muon fake factor is measured to be $0.14 \pm 0.03$, while the electron fake factor is $0.48 \pm 0.07$, where the uncertainties are statistical only. A systematic uncertainty of 35\% (56\%) in the electron (muon) fake factor is estimated from complementary control samples with low jet multiplicity or by applying a different selection to vary the fraction of jets containing heavy-flavour hadrons. The uncertainty in the muon fake factor is larger than in the electron fake factor due lower number of data events available for those checks. The fakelepton contributions in the nominal region (signal or preselection, denoted collectively by the superscript $R$ ) are obtained by multiplying the fake factors by the number of events in a region with the same selection as the nominal region, but where at least one lepton is of type $\ell$ : 

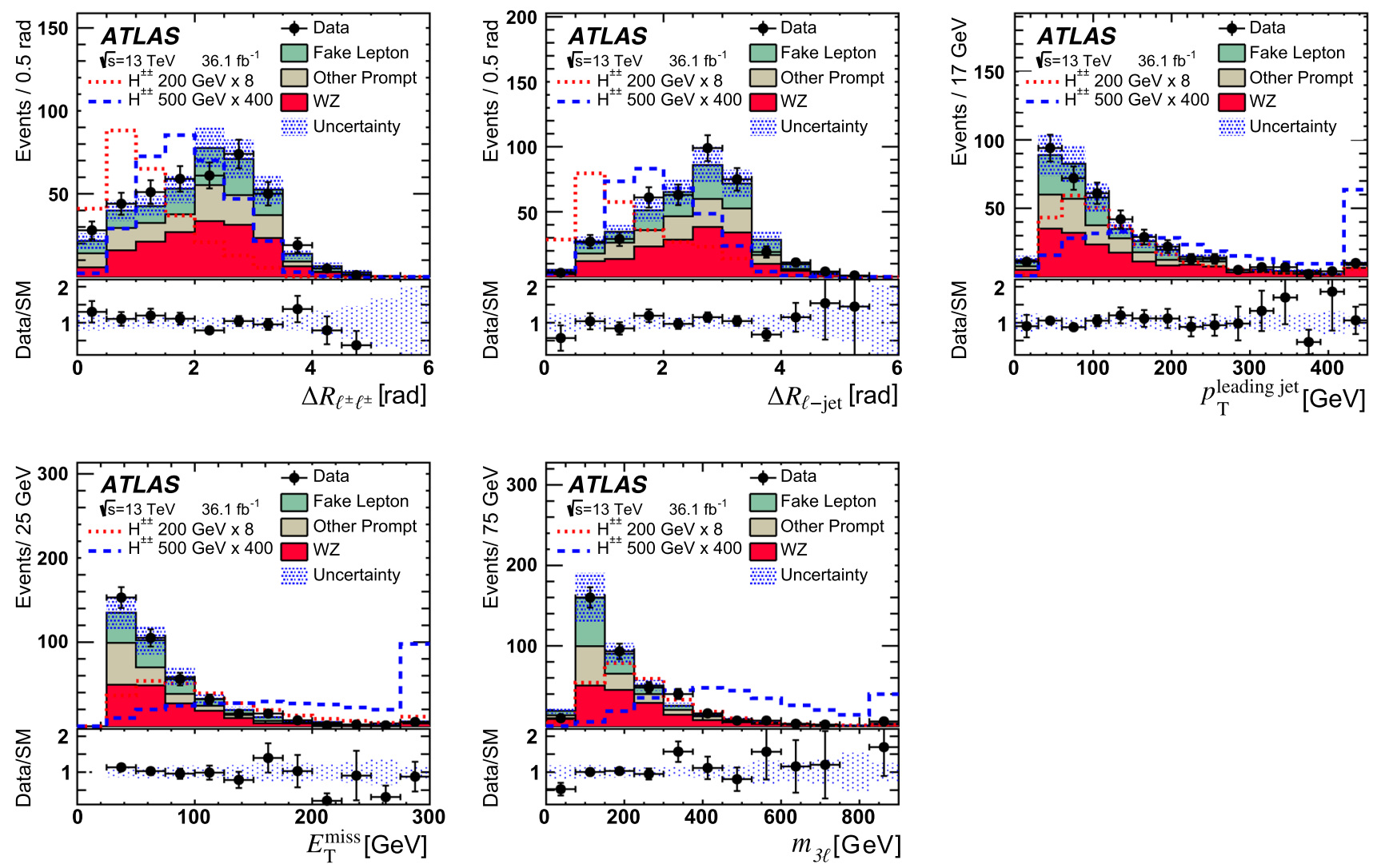

Fig. 2 Distribution of variables used for the signal region optimisation of the $3 \ell$ channel (a detailed description can be found in the caption of Fig. 1)

$$
\begin{aligned}
N_{e \mu}^{\mathrm{fake}, R}= & \theta_{e}^{2 \ell^{\mathrm{ss}}} \times\left(N^{\text {Data }}-N^{\text {Prompt }}-N^{\varnothing}\right)_{\mu \phi}^{R} \\
& +\theta_{\mu}^{2 \ell^{\mathrm{ss}}} \times\left(N^{\text {Data }}-N^{\text {Prompt }}-N^{\varnothing}\right)_{e \mu}^{R}, \\
N_{e e, \mu \mu}^{\mathrm{fake}, R}= & \theta_{e, \mu}^{2 \ell^{\mathrm{ss}}} \times\left(N^{\text {Data }}-N^{\text {Prompt }}-N^{\varnothing}\right)_{e \phi, \mu \mu}^{R} .
\end{aligned}
$$

where the prompt-lepton and the charge misidentifications contributions are subtracted as explained above.

Fake-lepton contribution estimate for the $3 \ell$ channel $\mathrm{A}$ method similar to that employed for the $2 \ell^{\mathrm{ss}}$ channel is applied for the $3 \ell$ channel. Here the opposite-sign lepton $\ell_{0}$ is assumed to be prompt, an assumption that was found to be valid in MC simulation. The fake-enriched region used to calculate the fake factors for the $3 \ell$ channel, which is described in Table 3 , follows the $3 \ell$ preselection conditions except that the jet multiplicity is required to be exactly one. The fake factors for electrons and muons are both calculated by applying a formula analogous to Eq. (1) to the $\ell_{0} e e / \ell_{0} e \notin$ and $\ell_{0} \mu \mu / \ell_{0} \mu \mu /$ regions, respectively. The muon fake factor is found to be $0.17 \pm 0.06$ and the electron fake factor is found to be $0.39 \pm 0.07$, where the errors are statistical only. The values are compatible with those obtained for the $2 \ell^{\text {ss }}$ channel. Additional control samples, defined such that the content is enriched in either $Z+$ jets or $t \bar{t}$ events, are used to test the method and to estimate systematic uncertainties of $55 \%$ and $81 \%$ for the electron and muon fake factors, respectively. The fake-lepton contributions to the nominal regions are then calculated using relations analogous to Eq. (3).

Fake-lepton contribution estimate for the $4 \ell$ channel There are too few data events to apply the fake-factor method in the $4 \ell$ channel. Instead, the fake-lepton contribution is estimated from the yields predicted by the MC simulation but corrected using process-dependent scale factors that are extracted in two fake-enriched control regions. The fakelepton contribution in this channel comes mainly from $t \bar{t} V$ processes, where the fake lepton originates from a $b$-jet. A small component from light quarks is also present. Two data samples designed to contain fake leptons originating from $Z+j$ ets and $t \bar{t}$ events are used to study the capability of the simulation to describe fake leptons originating from lightand heavy-flavour jets, respectively. The two control samples are labelled $\mathrm{Z}$ and $\mathrm{T}$ and are defined in Table 3. The samples are required to have three identified leptons. For the $Z$ region, the fake-lepton candidate is assumed not to be part of the lepton pair forming the $Z$ boson candidate. For the $\mathrm{T}$ region, the fake lepton is assumed to be the lepton with the lower $p_{\mathrm{T}}$ in the same-sign lepton pair. The scale fac- 

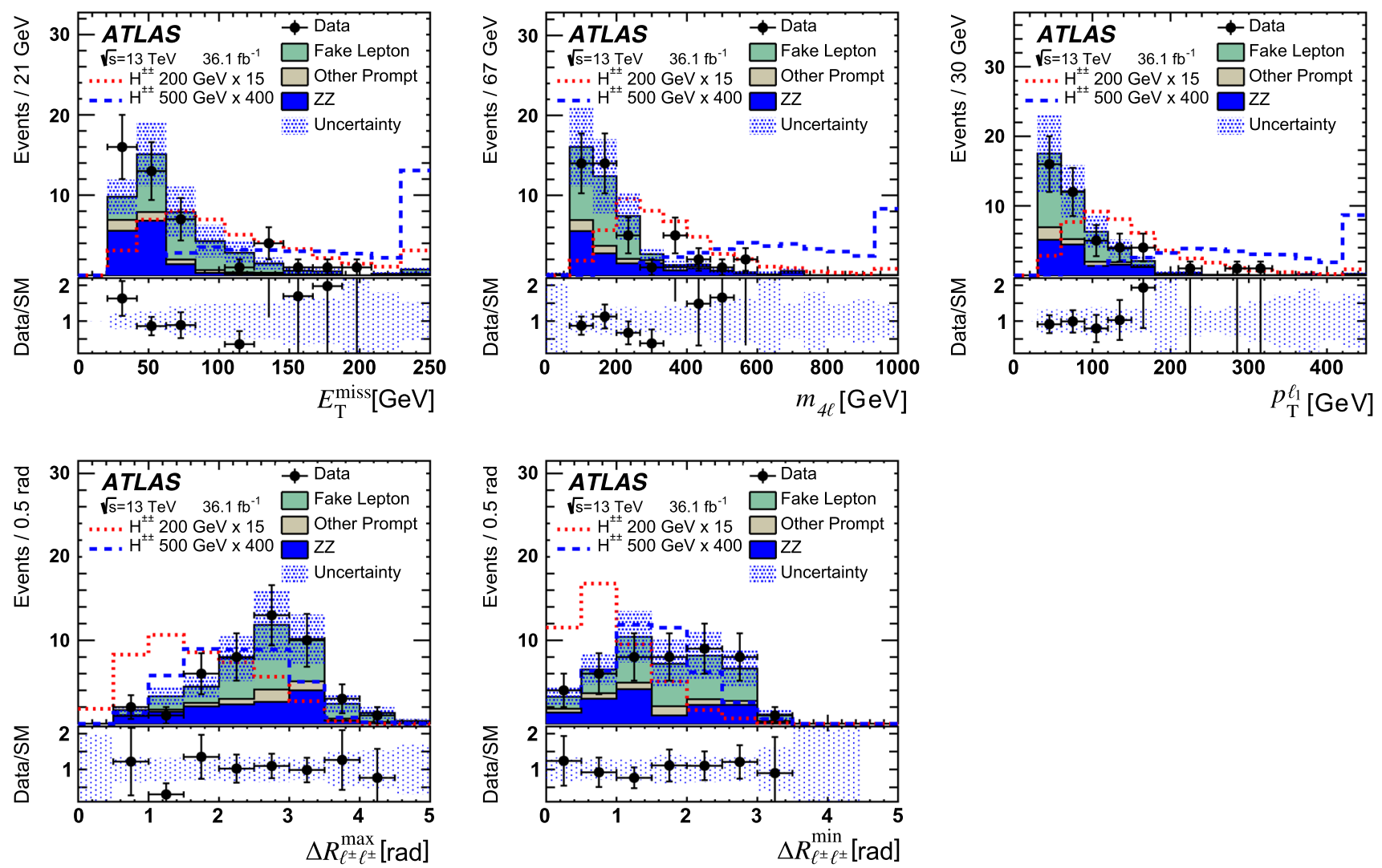

Fig. 3 Distribution of variables used for the signal region optimisation of the $4 \ell$ channel (a detailed description can be found in the caption of Fig. 1)

tors are derived independently for fake electrons and fake muons. Four scale factors $\lambda_{\mathrm{X}}^{\ell}$ (with $\ell=e, \mu$ and $\mathrm{X}=\mathrm{Z}, \mathrm{T}$ ) are obtained by solving the system of equations

$N_{\text {Data } \mid \mathrm{X}}^{\ell}-N_{\text {Prompt } \mid \mathrm{X}}^{\ell}=\lambda_{\mathrm{T}}^{\ell} N_{t \bar{t} \mid \mathrm{X}}^{\ell}+\lambda_{\mathrm{Z}}^{\ell} N_{Z+\text { jets } \mid \mathrm{X}}^{\ell}$,

where the event yields $N^{\ell}$ are labelled by the nature of the contribution, data (Data) or simulation (Prompt, $t \bar{t}$ and $Z+$ jets), and the equations are derived in each of the respective control region $\mathrm{X}(\mathrm{Z}$ or $\mathrm{T})$. The obtained scale factors are $\lambda_{\mathrm{T}}^{e}=1.12 \pm 0.05, \lambda_{\mathrm{Z}}^{e}=1.02 \pm 0.07, \lambda_{\mathrm{T}}^{\mu}=1.11 \pm 0.05$ and $\lambda_{\mathrm{Z}}^{\mu}=0.94 \pm 0.07$, where the errors are statistical only. Alternative trilepton control samples, where the jet multiplicity and the lepton $p_{\mathrm{T}}$ threshold are varied, are used to estimate a systematic uncertainty of $50 \%$ in these scale factors. The scale factors are used as weights to the simulated events that contain a fake lepton according to the fake-lepton flavour and the presence of heavy-flavour jets in the event.

\section{Signal region optimisation}

The hypothetical signal produces four $W^{ \pm}$bosons in each event. Since at least two leptonic $W$ boson decays are needed to lead to the multi-lepton topologies considered in this analysis, all signal events are expected to feature significant $E_{\mathrm{T}}^{\text {miss }}$, while jets are expected from hadronic $W$ boson decays for $2 \ell^{\text {ss }}$ and $3 \ell$ channels. Moreover, when the mass of the doubly charged Higgs boson is in the range of 200-300 GeV, each $H^{ \pm \pm}$is produced with a significant momentum and the two subsequent $W$ bosons are emitted close to each other in the laboratory frame. Consequently, the two same-sign leptons from the decays of the two $W$ bosons tend to be close in the $\eta-\phi$ plane. The decay products of the other doubly charged Higgs boson are generally well-separated from the two same-sign leptons.

The analysis channels face different background contributions from the SM. The $2 \ell^{\mathrm{ss}}$ category is populated with events containing one prompt lepton from a $W$ boson, or to a lesser extent from a $Z$ boson, and one fake lepton from the hadronic final state produced. The $2 \ell^{\mathrm{ss}}$ events with two same-sign electrons can also originate from Drell-Yan and $t \bar{t}$ production, where the charge of one of the electrons is misidentified, as explained above. In the $2 \ell^{s s}$ and $3 \ell$ channels, most of the expected prompt-lepton contribution is due to the production of $W Z$ associated with jets, with both bosons subsequently decaying into leptons. This process also produces other features of the signal, such as significant $E_{\mathrm{T}}^{\text {miss }}$ and the absence 
Table 4 The selection criteria used to define the signal regions. The variables are described in Sect. 5

\begin{tabular}{|c|c|c|c|c|c|c|}
\hline \multirow[t]{2}{*}{ Selection criteria } & \multicolumn{3}{|l|}{$2 \ell^{s s}$} & \multicolumn{2}{|l|}{$3 \ell$} & \multirow[t]{2}{*}{$4 \ell$} \\
\hline & $e^{ \pm} e^{ \pm}$ & $e^{ \pm} \mu^{ \pm}$ & $\mu^{ \pm} \mu^{ \pm}$ & SFOS 0 & SFOS 1,2 & \\
\hline \multicolumn{7}{|l|}{$m_{H^{ \pm \pm}}=200 \mathrm{GeV}$} \\
\hline$E_{\mathrm{T}}^{\mathrm{miss}}[\mathrm{GeV}]$ & $>100$ & $>100$ & $>100$ & $>45$ & $>45$ & $>60$ \\
\hline$m_{x \ell}[\mathrm{GeV}]$ & {$[25,130]$} & {$[15,150]$} & {$[35,150]$} & $>160$ & $>170$ & $>230$ \\
\hline$\Delta R_{\ell^{ \pm} \ell^{ \pm}}[\mathrm{rad}]$. & $<0.8$ & $<1.8$ & $<0.9$ & {$[0.15,1.57]$} & {$[0.00,1.52]$} & \\
\hline$\Delta \phi\left(\ell \ell, E_{\mathrm{T}}^{\text {miss }}\right)$ [rad.] & $<1.1$ & $<1.3$ & $<1.3$ & & & \\
\hline$S$ [rad.] & $<0.3$ & $<0.3$ & $<0.2$ & & & \\
\hline$m_{\text {jets }}[\mathrm{GeV}]$ & {$[140,770]$} & {$[95,330]$} & {$[95,640]$} & & & \\
\hline$\Delta R_{\ell-\text { jet }}[\mathrm{rad}]$. & & & & {$[0.08,1.88]$} & {$[0.07,1.31]$} & \\
\hline$p_{\mathrm{T}}^{\text {leading jet }}[\mathrm{GeV}]$ & & & & $>80$ & $>55$ & \\
\hline$p_{\mathrm{T}}^{\ell_{1}}[\mathrm{GeV}]$ & & & & & & $>65$ \\
\hline$\Delta R_{\ell^{ \pm} \ell^{ \pm}}^{\min }[\mathrm{rad}]$. & & & & & & {$[0.16,1.21]$} \\
\hline$\Delta R_{\ell^{ \pm} \ell^{ \pm}}^{\max }[\mathrm{rad}]$. & & & & & & {$[0.27,2.03]$} \\
\hline \multicolumn{7}{|l|}{$m_{H^{ \pm \pm}}=300 \mathrm{GeV}$} \\
\hline$E_{\mathrm{T}}^{\mathrm{miss}}[\mathrm{GeV}]$ & $>200$ & $>200$ & $>200$ & $>65$ & $>55$ & $>60$ \\
\hline$m_{x \ell}[\mathrm{GeV}]$ & {$[105,340]$} & {$[80,320]$} & {$[80,320]$} & $>170$ & $>210$ & $>270$ \\
\hline$\Delta R_{\ell^{ \pm} \ell^{ \pm}}[\mathrm{rad}]$. & $<1.4$ & $<1.8$ & $<1.8$ & {$[0.18,2.23]$} & {$[0.08,2.23]$} & \\
\hline$\Delta \phi\left(\ell \ell, E_{\mathrm{T}}^{\text {miss }}\right)[\mathrm{rad}]$. & $<2.1$ & $<2.4$ & $<2.4$ & & & \\
\hline$S$ [rad.] & $<0.4$ & $<0.4$ & $<0.4$ & & & \\
\hline$m_{\text {jets }}[\mathrm{GeV}]$ & {$[180,770]$} & {$[130,640]$} & {$[130,640]$} & & & \\
\hline$\Delta R_{\ell j}[\mathrm{rad}]$. & & & & {$[0.27,2.37]$} & {$[0.21,2.08]$} & \\
\hline$p_{\mathrm{T}}^{\text {leading jet }}[\mathrm{GeV}]$ & & & & $>95$ & $>80$ & \\
\hline$p_{\mathrm{T}}^{\ell_{1}}[\mathrm{GeV}]$ & & & & & & $>45$ \\
\hline$\Delta R_{\ell^{ \pm} \ell^{ \pm}}^{\min }[\mathrm{rad}]$. & & & & & & {$[0.09,1.97]$} \\
\hline$\Delta R_{\ell^{ \pm} \ell^{ \pm}}^{\max }[\mathrm{rad}]$. & & & & & & {$[0.44,2.68]$} \\
\hline \multicolumn{7}{|l|}{$m_{H^{ \pm \pm}}=400 \mathrm{GeV}$} \\
\hline$E_{\mathrm{T}}^{\text {miss }}[\mathrm{GeV}]$ & $>200$ & $>200$ & $>200$ & $>65$ & $>85$ & $>60$ \\
\hline$m_{x \ell}[\mathrm{GeV}]$ & {$[105,340]$} & {$[80,350]$} & {$[80,350]$} & $>230$ & $>250$ & $>270$ \\
\hline$\Delta R_{\ell^{ \pm} \ell^{ \pm}}[\mathrm{rad}]$. & $<2.2$ & $<1.8$ & $<1.8$ & {$[0.22,2.39]$} & {$[0.29,2.69]$} & \\
\hline$\Delta \phi\left(\ell \ell, E_{\mathrm{T}}^{\mathrm{miss}}\right)[\mathrm{rad}]$. & $<2.4$ & $<2.4$ & $<2.4$ & & & \\
\hline$S$ [rad.] & $<0.6$ & $<0.6$ & $<0.5$ & & & \\
\hline$m_{\text {jets }}[\mathrm{GeV}]$ & {$[280,1200]$} & {$[220,1200]$} & {$[220,1200]$} & & & \\
\hline$\Delta R_{\ell j}[\mathrm{rad}]$. & & & & {$[0.30,2.59]$} & {$[0.31,2.30]$} & \\
\hline$p_{\mathrm{T}}^{\text {leading jet }}[\mathrm{GeV}]$ & & & & $>120$ & $>100$ & \\
\hline$p_{\mathrm{T}}^{\ell_{1}}[\mathrm{GeV}]$ & & & & & & $>110$ \\
\hline$\Delta R_{\ell^{ \pm} \ell^{ \pm}}^{\min }[\mathrm{rad}]$. & & & & & & {$[0.39,2.22]$} \\
\hline$\Delta R_{\ell^{ \pm} \ell^{ \pm}}^{\max }[\mathrm{rad}]$. & & & & & & {$[0.55,2.90]$} \\
\hline
\end{tabular}


Table 4 continued

\begin{tabular}{|c|c|c|c|c|c|c|}
\hline \multirow[t]{2}{*}{ Selection criteria } & \multicolumn{3}{|l|}{$2 \ell^{\mathrm{ss}}$} & \multicolumn{2}{|l|}{$3 \ell$} & \multirow[t]{2}{*}{$4 \ell$} \\
\hline & $e^{ \pm} e^{ \pm}$ & $e^{ \pm} \mu^{ \pm}$ & $\mu^{ \pm} \mu^{ \pm}$ & SFOS 0 & SFOS 1,2 & \\
\hline \multicolumn{7}{|l|}{$m_{H^{ \pm \pm}}=500-700 \mathrm{GeV}$} \\
\hline$E_{\mathrm{T}}^{\mathrm{miss}}[\mathrm{GeV}]$ & $>250$ & $>250$ & $>250$ & $>120$ & $>100$ & $>60$ \\
\hline$m_{x \ell}[\mathrm{GeV}]$ & {$[105,730]$} & {$[110,440]$} & {$[110,440]$} & $>230$ & $>300$ & $>370$ \\
\hline$\Delta R_{\ell^{ \pm} \ell^{ \pm}}[\mathrm{rad}]$. & $<2.6$ & $<2.2$ & $<2.2$ & {$[0.39,3.11]$} & {$[0.29,2.85]$} & \\
\hline$\Delta \phi\left(\ell \ell, E_{\mathrm{T}}^{\mathrm{miss}}\right)[\mathrm{rad}]$. & $<2.6$ & $<2.4$ & $<2.4$ & & & \\
\hline$S$ [rad.] & $<1.1$ & $<1.1$ & $<1.1$ & & & \\
\hline$m_{\text {jets }}[\mathrm{GeV}]$ & $>440$ & $>470$ & $>470$ & & & \\
\hline$\Delta R_{\ell j}[\mathrm{rad}]$. & & & & {$[0.60,2.68]$} & {$[0.31,2.53]$} & \\
\hline$p_{\mathrm{T}}^{\text {leading jet }}[\mathrm{GeV}]$ & & & & $>130$ & $>130$ & \\
\hline$p_{\mathrm{T}}^{\ell_{1}}[\mathrm{GeV}]$ & & & & & & $>160$ \\
\hline$\Delta R_{\ell^{ \pm} \ell^{ \pm}}^{\min }[\mathrm{rad}]$. & & & & & & {$[0.53,3.24]$} \\
\hline$\Delta R_{\ell^{ \pm} \ell^{ \pm}}^{\max }[\mathrm{rad}]$. & & & & & & {$[0.59,2.94]$} \\
\hline
\end{tabular}

of $b$-jets for most of the production cross-section. For the $W Z$ events, the mass of the same-flavour opposite-sign lepton pair is close to the $Z$ boson mass, while no such resonant distribution is expected for the signal. In the $4 \ell$ channel, the dominant background originates from $t \bar{t} V$ and $Z Z$ production. Processes containing top quarks $(t \bar{t}, t \bar{t} V)$ can lead to events with multiple leptons in the final state. A noticeable feature of those processes is the presence of $b$-jets.

Given these properties of the signal and of the expected background, the following discriminating variables, in addition to $E_{\mathrm{T}}^{\text {miss }}$, are considered:

- $m_{x \ell}$, the invariant mass of the system composed of all selected leptons in the event, where $x$ can be 2, 3 or 4 .

- $\Delta R_{\ell^{ \pm} \ell^{ \pm}}$, the distance in $\eta-\phi$ between two same-sign leptons. This variable is used for the $2 \ell^{\mathrm{ss}}$ and $3 \ell$ channels. In the $4 \ell$ channel, two such variables can be calculated per event, $\Delta R_{\ell^{ \pm} \ell^{ \pm}}^{\min }$ and $\Delta R_{\ell^{ \pm} \ell^{ \pm}}^{\max }$, denoting the minimum and maximum values, respectively.

- $m_{\text {jets }}$, the invariant mass of the system composed of all jets in the event. When there are more than four jets in the event, only the leading four jets are used. This variable is used only for the $2 \ell^{\mathrm{ss}}$ channel.

- $p_{\mathrm{T}}^{\text {leading jet }}$, the transverse momentum of the highest- $p_{\mathrm{T}}$ jet.

- $\Delta \phi\left(\ell \ell, E_{\mathrm{T}}^{\mathrm{miss}}\right)$, the difference in azimuth between the dilepton system and $E_{\mathrm{T}}^{\text {miss }}$. This variable is used in the $2 \ell^{\text {ss }}$ channel.

- $\Delta R_{\ell-\text { jet }}$, the minimal distance in $\eta-\phi$ between any lepton and its closest jet. This variable is used in the $3 \ell$ channel.

- $S$, is a variable used for the $2 \ell^{\mathrm{ss}}$ channel to describe the event topology in the transverse plane, and defined using the spread of the $\phi$ angles of the leptons, $E_{\mathrm{T}}^{\text {miss }}$, and jets as follows:

$$
S=\frac{\mathcal{R}\left(\phi_{\ell_{1}}, \phi_{\ell_{2}}, \phi_{E_{\mathrm{T}}^{\mathrm{miss}}}\right) \cdot \mathcal{R}\left(\phi_{j 1}, \phi_{j 2}, \ldots\right)}{\mathcal{R}\left(\phi_{\ell_{1}}, \phi_{\ell_{2}}, \phi_{E_{\mathrm{T}}^{\mathrm{miss}}}, \phi_{j 1}, \phi_{j 2}, \ldots\right)}
$$

where the $\mathcal{R}$ is the root mean square that quantifies the spread, $\mathcal{R}\left(\phi_{1}, \ldots, \phi_{n}\right)=\sqrt{\frac{1}{n} \sum_{i=1}^{n}\left(\phi_{i}-\bar{\phi}\right)^{2}}$. The azimuthal angles $\phi$ are bounded in $(-\pi, \pi]$, and the bound is considered in the calculation. The $S$ variable is expected to be on average smaller for the signal than for the background for low $H^{ \pm \pm}$mass values.

The distributions of the selected variables for the $2 \ell^{\mathrm{ss}}, 3 \ell$ and $4 \ell$ channels are shown at preselection level in Figs. 1, 2 and 3 , respectively. The data are compared with the sum of the prompt lepton, fake lepton and charge-misidentified lepton background predictions. The prompt-lepton backgrounds are estimated with simulations while the background from fake leptons and charge-flipped leptons are measured with the methods described in the previous section. Good agreement is observed in both normalisation and shape, demonstrating that the background contributions are well modelled. The expected signal distributions for various $H^{ \pm \pm}$masses are also shown to illustrate the discriminating power of the selected variables.

The strategy used to extract the signal is based on rectangular cut optimisation using the TMVA package [59]. For each $m_{H^{ \pm \pm}}$hypothesis, six signal regions are defined using the following lepton flavour content: in the $2 \ell^{\text {ss }}$ channel, three signal regions are optimised separately for $e e, e \mu$ and $\mu \mu$ channels; in the $3 \ell$ channel, the signal regions are optimised 


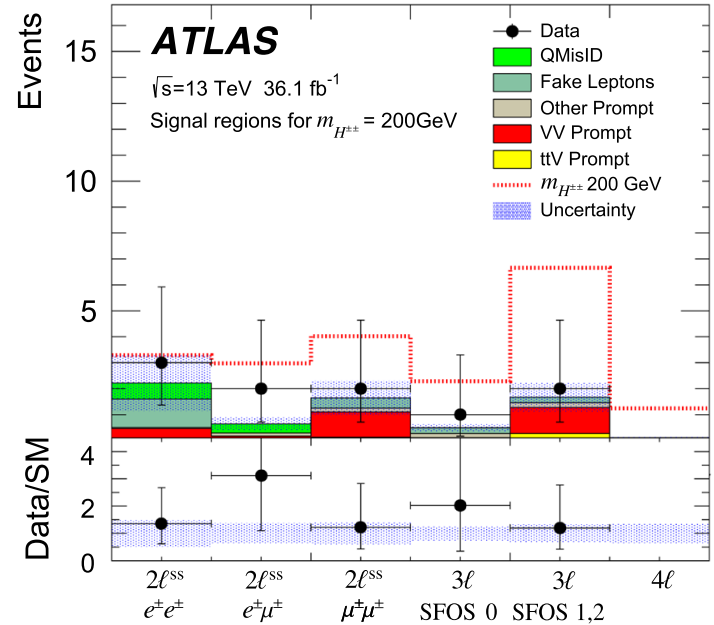

(a) $200 \mathrm{GeV}$

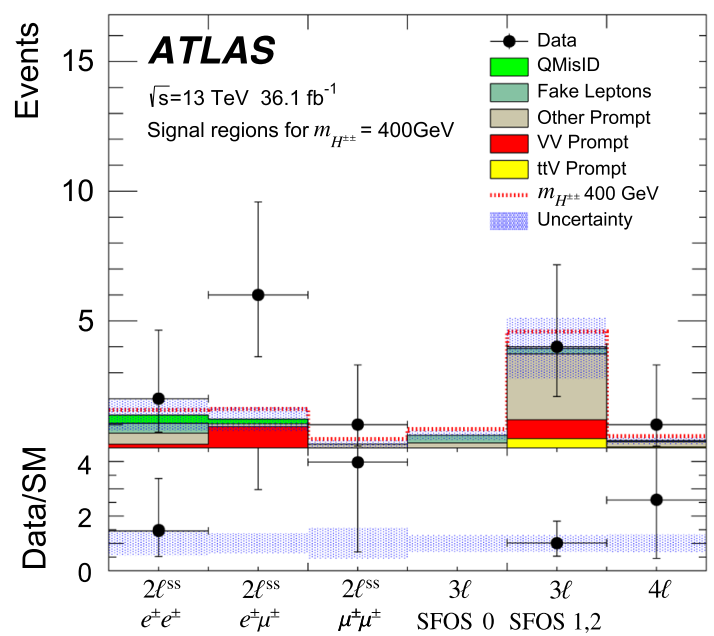

(c) $400 \mathrm{GeV}$

Fig. 4 Event yields in the signal regions optimised for the $m_{H^{ \pm \pm}}=200$, 300,400 and $500 \mathrm{GeV}$ searches. The bottom panel shows the ratio of the data to the total background prediction, where the band illustrates the total uncertainty of the SM background. The error bars attributed

separately for events with no same-flavour opposite-sign lepton pairs (SFOS 0, for which the SM background is small) and for events with one or two such pairs (SFOS 1,2); the $4 \ell$ channel is treated globally, with no further lepton flavour distinction. The selection criteria used to define the signal regions are shown in Table 4 . The optimisation is performed as a function of the $H^{ \pm \pm}$mass for $m_{H^{ \pm \pm}}=200,300,400$ and $500 \mathrm{GeV}$, and seeks the best expected signal significance. The last optimisation point is applied to $m_{H^{ \pm \pm}} \geq 600 \mathrm{GeVas}$ well, since the signal discrimination power does not vary significantly in this regime.

In order to verify the background estimate reliability for the signal region, three further checks were performed:

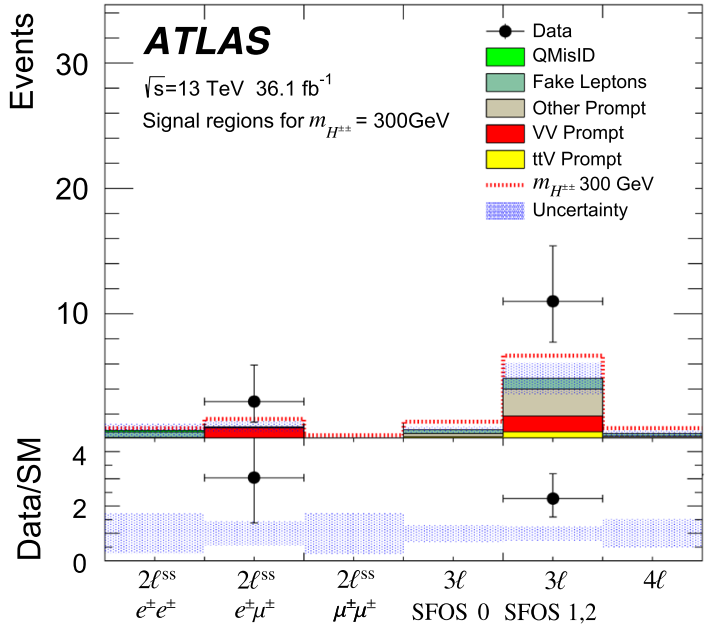

(b) $300 \mathrm{GeV}$

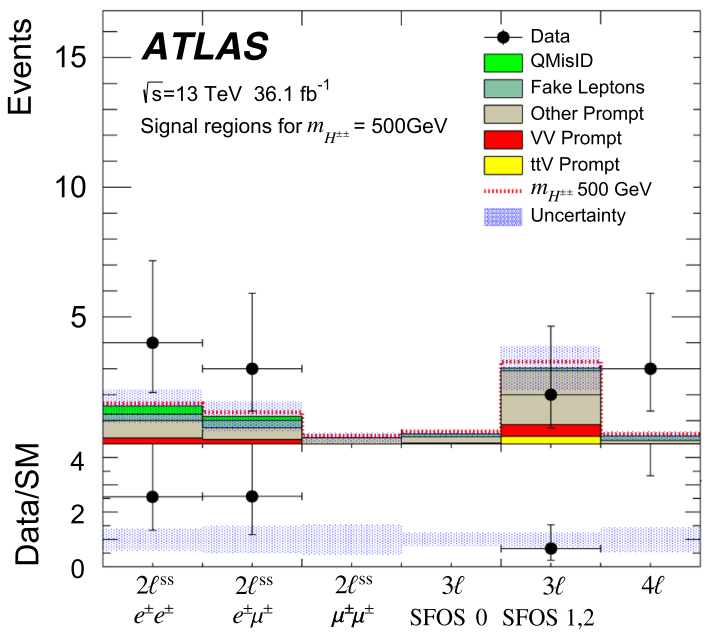

(d) $500 \mathrm{GeV}$

to data are estimated assuming a Poisson distribution with the average equal to the respective yields. The signal prediction is represented as a dotted histogram, stacked on the SM background

the optimised cuts were applied individually, the cuts were applied successively, or each cut was inverted while the other cuts were applied. The agreement between data and prediction remains adequate for all those cases.

\section{Systematic uncertainties}

The theoretical uncertainties associated with the signal prediction originate from the PDFs, the matrix element calculation and the parton shower simulation. The uncertainties related to PDFs are evaluated using the Hessian method provided in LHAPDF6 [60] and are found to be in the range 
Table 5 Event yields in the signal regions of corresponding targeted masses of $H^{ \pm \pm}$. The signal yield is for the corresponding mass point and is normalised to the luminosity of $36.1 \mathrm{fb}^{-1}$. The dominant background from prompt-lepton sources is from the $W Z$ process in the $2 \ell^{s s}$ channel. For the $3 \ell$ and $4 \ell$ channels, the dominant background from prompt-lepton sources is from $W Z$ and $t \bar{t} V$ processes. The overall signal acceptance $A$ and the upper limit of extra contribution to each signal region at $95 \%$ confidence level $n_{95}$ are also presented. The data and SM prediction yields obtained for $m_{H^{ \pm \pm}}=500 \mathrm{GeV}$ are also valid for $m_{H^{ \pm \pm}}=600$ and $700 \mathrm{GeV}$

\begin{tabular}{|c|c|c|c|c|c|c|}
\hline \multirow[t]{2}{*}{ Subchannel } & \multicolumn{3}{|l|}{$2 \ell^{s s}$} & \multicolumn{2}{|l|}{$3 \ell$} & \multirow[t]{2}{*}{$4 \ell$} \\
\hline & $\overline{e^{ \pm} e^{ \pm}}$ & $e^{ \pm} \mu^{ \pm}$ & $\mu^{ \pm} \mu^{ \pm}$ & SFOS 0 & SFOS 1,2 & \\
\hline \multicolumn{7}{|l|}{$m_{H^{ \pm \pm}}=200 \mathrm{GeV}$} \\
\hline Prompt lepton & $0.5 \pm 0.2$ & $0.3 \pm 0.2$ & $1.3 \pm 0.6$ & $0.3 \pm 0.1$ & $1.4 \pm 0.5$ & $0.07 \pm 0.03$ \\
\hline QMisID & $0.6 \pm 0.2$ & $0.4 \pm 0.1$ & - & - & - & - \\
\hline Fake lepton & $1 \pm 1$ & $<0.4$ & $0.4 \pm 0.3$ & $0.2 \pm 0.1$ & $0.2 \pm 0.1$ & $0.03 \pm 0.02$ \\
\hline Total background & $2 \pm 1$ & $0.6 \pm 0.3$ & $1.7 \pm 0.7$ & $0.5 \pm 0.1$ & $1.7 \pm 0.6$ & $0.11 \pm 0.05$ \\
\hline Signal & $1.1 \pm 0.2$ & $2.3 \pm 0.4$ & $2.4 \pm 0.4$ & $1.8 \pm 0.3$ & $5.0 \pm 0.9$ & $1.1 \pm 0.2$ \\
\hline$A[\%]$ & 0.037 & 0.080 & 0.082 & 0.061 & 0.17 & 0.038 \\
\hline$n_{95}$ & 12.3 & 7.1 & 7.5 & 4.1 & 7.7 & 3.8 \\
\hline Data & 3 & 2 & 2 & 1 & 2 & 0 \\
\hline \multicolumn{7}{|l|}{$m_{H^{ \pm \pm}}=300 \mathrm{GeV}$} \\
\hline Prompt lepton & $0.1 \pm 0.1$ & $0.9 \pm 0.4$ & $0.02 \pm 0.02$ & $0.4 \pm 0.1$ & $4 \pm 1$ & $0.3 \pm 0.1$ \\
\hline QMisID & $0.1 \pm 0.1$ & $0.07 \pm 0.04$ & - & - & - & - \\
\hline Fake lepton & $0.4 \pm 0.5$ & $<0.2$ & $<0.4$ & $0.3 \pm 0.2$ & $0.8 \pm 0.4$ & $0.2 \pm 0.2$ \\
\hline Total background & $0.7 \pm 0.5$ & $1.0 \pm 0.5$ & $0.02 \pm 0.02$ & $0.8 \pm 0.2$ & $5 \pm 2$ & $0.5 \pm 0.2$ \\
\hline Signal & $0.16 \pm 0.03$ & $0.6 \pm 0.1$ & $0.29 \pm 0.05$ & $0.6 \pm 0.1$ & $1.8 \pm 0.3$ & $0.43 \pm 0.08$ \\
\hline$A[\%]$ & 0.027 & 0.10 & 0.049 & 0.11 & 0.30 & 0.071 \\
\hline$n_{95}$ & 4.0 & 9.6 & 3.0 & 3.1 & 22.7 & 3.8 \\
\hline Data & 0 & 3 & 0 & 0 & 11 & 0 \\
\hline \multicolumn{7}{|l|}{$m_{H^{ \pm \pm}}=400 \mathrm{GeV}$} \\
\hline Prompt lepton & $0.7 \pm 0.3$ & $1.0 \pm 0.4$ & $0.2 \pm 0.1$ & $0.3 \pm 0.1$ & $4 \pm 1$ & $0.3 \pm 0.1$ \\
\hline QMisID & $0.3 \pm 0.1$ & $0.2 \pm 0.1$ & - & - & - & - \\
\hline Fake lepton & $0.4 \pm 0.5$ & $<0.3$ & $<0.4$ & $0.3 \pm 0.2$ & $0.2 \pm 0.1$ & $0.05 \pm 0.04$ \\
\hline Total background & $1.4 \pm 0.6$ & $1.2 \pm 0.5$ & $0.3 \pm 0.1$ & $0.6 \pm 0.2$ & $4 \pm 1$ & $0.4 \pm 0.1$ \\
\hline Signal & $0.20 \pm 0.04$ & $0.38 \pm 0.07$ & $0.19 \pm 0.03$ & $0.23 \pm 0.04$ & $0.6 \pm 0.1$ & $0.17 \pm 0.03$ \\
\hline$A[\%]$ & 0.11 & 0.21 & 0.11 & 0.13 & 0.36 & 0.092 \\
\hline$n_{95}$ & 10.4 & 18.3 & 6.4 & 3.1 & 10.4 & 4.3 \\
\hline Data & 2 & 6 & 1 & 0 & 4 & 1 \\
\hline \multicolumn{7}{|l|}{$m_{H^{ \pm \pm}}=500 \mathrm{GeV}$} \\
\hline Prompt lepton & $1.0 \pm 0.4$ & $0.7 \pm 0.3$ & $0.3 \pm 0.2$ & $0.4 \pm 0.1$ & $3 \pm 1$ & $0.2 \pm 0.1$ \\
\hline QMisID & $0.3 \pm 0.1$ & $0.2 \pm 0.1$ & - & - & - & - \\
\hline Fake lepton & $0.2 \pm 0.5$ & $0.3 \pm 0.5$ & $<0.4$ & $0.11 \pm 0.06$ & $0.10 \pm 0.05$ & $0.2 \pm 0.2$ \\
\hline Total background & $1.6 \pm 0.6$ & $1.2 \pm 0.6$ & $0.3 \pm 0.2$ & $0.5 \pm 0.1$ & $3.0 \pm 0.8$ & $0.4 \pm 0.2$ \\
\hline Signal & $0.10 \pm 0.02$ & $0.16 \pm 0.03$ & $0.07 \pm 0.01$ & $0.09 \pm 0.02$ & $0.24 \pm 0.04$ & $0.06 \pm 0.01$ \\
\hline$A[\%]$ & 0.16 & 0.25 & 0.11 & 0.14 & 0.37 & 0.098 \\
\hline$A[\%] m_{H^{ \pm \pm}}=600 \mathrm{GeV}$ & 0.22 & 0.36 & 0.16 & 0.17 & 0.44 & 0.11 \\
\hline$A[\%] m_{H^{ \pm \pm}}=700 \mathrm{GeV}$ & 0.26 & 0.38 & 0.17 & 0.19 & 0.48 & 0.12 \\
\hline$n_{95}$ & 8.6 & 12.7 & 3.8 & 3.0 & 7.9 & 4.9 \\
\hline Data & 4 & 3 & 0 & 0 & 2 & 3 \\
\hline
\end{tabular}


from $2.5 \%$ to $4.5 \%$. The uncertainty of the parton shower simulation is assessed by comparing PYTHIA (with A14 tune) and Herwig++ (with UEEE5 tune [61]), and is found to be $2.4 \%, 1.7 \%$, and $3.8 \%$ for the $2 \ell^{\mathrm{ss}}, 3 \ell$, and $4 \ell$ channels, respectively. The higher-order corrections are assumed to induce an additional $15 \%$ uncertainty in the cross-section calculations [33]. Combining those uncertainties in quadrature, an overall uncertainty of $17 \%$ is obtained for the signal normalisation.

The theoretical uncertainties associated with the largest SM backgrounds, $V V$ [48] (including same-sign $W W q q$ and $W Z$ processes) and $t \bar{t} V$ [62], are estimated using dedicated MC samples, where the factorisation and renormalisation scales are varied independently by factors of 2 and 0.5 and the parton shower parameters are varied within the given model uncertainties. The theoretical uncertainties obtained are $24 \%$ for $V V$ and $17 \%$ for $t \bar{t} V$. The uncertainties related to PDFs are found to be negligible. The uncertainty associated with the $V \gamma$ contributions is taken to be $25 \%$, as indicated by dedicated studies using converted photons. The uncertainties related to the $V V V$ and $t Z$ process predictions are taken from the respective inclusive cross-section measurements $[63,64]$. For other rare backgrounds which have no dedicated measurements yet $\left(t t \bar{t}, t \bar{t} W^{+} W^{-}\right)$, uncertainties of $50 \%$ are assumed and are found to have a negligible impact on the sensitivity.

The experimental uncertainties arise from the accuracy of the detector simulation and from the uncertainties associated with the data-driven methods that are used to estimate the instrumental backgrounds. These uncertainties originate from the following sources:

- The uncertainties related to event reconstruction include the lepton $[52,65]$ and the jet [66] energy scales and resolutions and the uncertainties in the reconstruction of $E_{\mathrm{T}}^{\mathrm{miss}}$ [58]. The impact of this type of uncertainty on the signal and background yields is in the range $3-8 \%$ and $10-30 \%$, respectively.

- The uncertainties related to the efficiencies of electron [51] and muon [52] reconstruction and identification, including the uncertainties in the trigger efficiency are estimated in dedicated studies. The impact of this type of uncertainty on the signal and background yields is found to be in the range $4-6 \%$ and $2-5 \%$, respectively. The uncertainties related to the $b$-jet identification algorithms, used in the analysis to veto events containing $b$ jets, are found to be negligible.

- The uncertainties originating from data-taking conditions include the luminosity measurement and the pile-up simulation procedure. The uncertainty of the integrated luminosity is $2.1 \%$, determined using a methodology similar to that detailed in Ref. [67]. The uncertainty from the pile-up simulation is about $5 \%$.

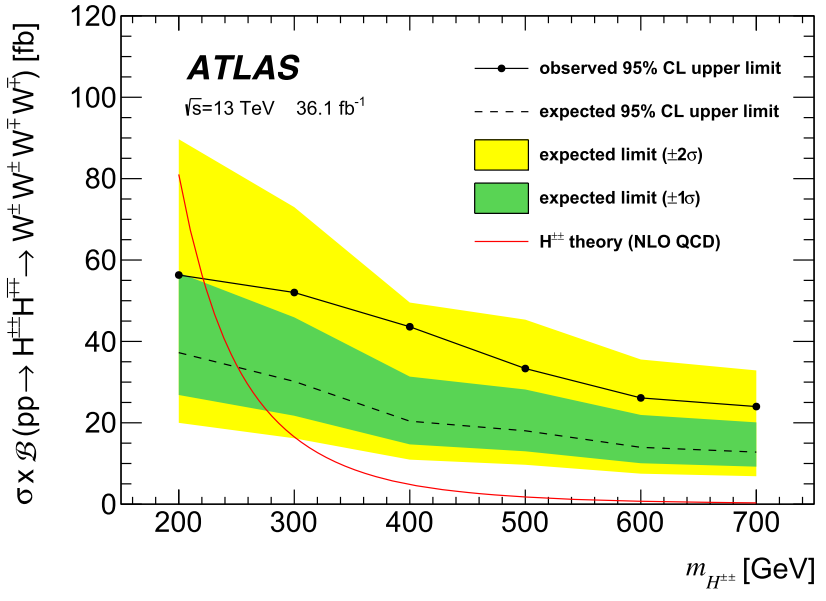

Fig. 5 Observed and expected upper limits for $p p \rightarrow H^{ \pm \pm} H^{\mp \mp} \rightarrow$ $W^{ \pm} W^{ \pm} W^{\mp} W^{\mp}$ cross-section times branching fraction at $95 \% \mathrm{CL}$ obtained from the combination of $2 \ell^{\mathrm{ss}}, 3 \ell$ and $4 \ell$ channels. The region above the observed limit is excluded by the measurement. The bands represent the expected exclusion curves within one and two standard deviations. The theoretical prediction [3] including the NLO QCD corrections [33] is also shown and is excluded for $m_{H^{ \pm \pm}}<220 \mathrm{GeV}$

- The uncertainties related to the background contributions from electron charge misidentification are $22-28 \%$ for the $2 \ell^{\mathrm{ss}} e e$ and $e \mu$ channels. The uncertainties of the fake-lepton contributions range from 50 to $250 \%$, and mainly originate from the fake factors $\left(2 \ell^{\mathrm{ss}}\right.$ and $3 \ell$ channels) and the scale factors for MC simulation ( $4 \ell$ channel) described in Sect. 4.3.2 and the statistics of the control samples. The uncertainties exceed $100 \%$ in some cases due to the subtraction of the prompt-lepton contributions in the fake-lepton control regions.

The theoretical and experimental systematic uncertainties described above are assumed to be correlated amongst the various signal regions in the interpretation of the final results. Overall, the sensitivity of the search is dominated by the statistical uncertainty of the event yield in the signal regions.

\section{Results}

The expected and observed event yields in the signal regions are shown in Fig. 4 and Table 5. For a $H^{ \pm \pm}$mass of $200 \mathrm{GeV}$, substantial signal yield is expected in all channels, and the analysis sensitivity is found to be comparable across the $2 \ell^{s s}, 3 \ell$ and $4 \ell$ channels. No significant excess has been observed. Table 5 also includes the overall signal acceptance $A$, defined as the number of selected events selected in a given channel divided by the total number of $p p \rightarrow H^{ \pm \pm} H^{\mp \mp} \rightarrow W^{ \pm} W^{ \pm} W^{\mp} W^{\mp}$ events and representing the signal reduction due to phase space acceptance, branching ratio and detector efficiency. 
The statistical analysis of the results is based on a likelihood ratio test [68] using the $\mathrm{CL}_{\mathrm{s}}$ method [69]. The parameter of interest is the signal strength, defined as the cross-section of the hypothetical contribution from physics beyond the SM in units of the cross-section of the benchmark model. The likelihood function is constructed from Poisson probability distributions of counting experiments for each of the six channels in each signal region. The systematic uncertainties are treated as nuisance parameters implemented in the likelihood functions with Gaussian constraints.

The expected and observed upper limits of the $H^{ \pm \pm} \rightarrow$ $W^{ \pm} W^{ \pm}$cross-section at $95 \%$ confidence level (CL), obtained from the combination of $2 \ell^{\mathrm{ss}}, 3 \ell$ and $4 \ell$ channels for the six $H^{ \pm \pm}$mass hypotheses are shown in Fig. 5. Assuming a linear interpolation of the sensitivity between neighbouring mass hypotheses, and the cross-section of the benchmark model, the observed (expected) lower limit on the mass of the $H^{ \pm \pm}$ boson is $220 \mathrm{GeV}(250 \mathrm{GeV})$ at $95 \% \mathrm{CL}$.

\section{Conclusion}

A search for the pair production of doubly charged Higgs scalar bosons with subsequent decays into $W$ bosons is performed in proton-proton collisions at a centre-of-mass energy of $13 \mathrm{TeV}$. The data sample was collected by the ATLAS experiment at the LHC and corresponds to an integrated luminosity of $36.1 \mathrm{fb}^{-1}$. The search for the $H^{ \pm \pm} \rightarrow$ $W^{ \pm} W^{ \pm}$decay mode, not considered in previous analyses at colliders, is motivated by a model with an extended scalar sector that includes a triplet in addition to the Standard Model scalar doublet. The analysis proceeds through the selection of multi-lepton events in three channels (a pair of same-sign leptons, three leptons and four leptons) with missing transverse momentum and jets. The signal region is optimised as a function of the $H^{ \pm \pm}$mass. The data are found to be in good agreement with the Standard Model predictions for all channels investigated. Combining those channels, the model considered is excluded at $95 \%$ confidence level for $H^{ \pm \pm}$ boson masses between 200 and $220 \mathrm{GeV}$.

Acknowledgements We thank CERN for the very successful operation of the LHC, as well as the support staff from our institutions without whom ATLAS could not be operated efficiently.

We acknowledge the support of ANPCyT, Argentina; YerPhI, Armenia; ARC, Australia; BMWFW and FWF, Austria; ANAS, Azerbaijan; SSTC, Belarus; CNPq and FAPESP, Brazil; NSERC, NRC and CFI, Canada; CERN; CONICYT, Chile; CAS, MOST and NSFC, China; COLCIENCIAS, Colombia; MSMT CR, MPO CR and VSC CR, Czech Republic; DNRF and DNSRC, Denmark; IN2P3-CNRS, CEADRF/IRFU, France; SRNSFG, Georgia; BMBF, HGF, and MPG, Germany; GSRT, Greece; RGC, Hong Kong SAR, China; ISF and Benoziyo Center, Israel; INFN, Italy; MEXT and JSPS, Japan; CNRST, Morocco; NWO, Netherlands; RCN, Norway; MNiSW and NCN, Poland; FCT, Portugal; MNE/IFA, Romania; MES of Russia and NRC KI, Russian Federation; JINR; MESTD, Serbia; MSSR, Slovakia; ARRS and MIZŠ,
Slovenia; DST/NRF, South Africa; MINECO, Spain; SRC and Wallenberg Foundation, Sweden; SERI, SNSF and Cantons of Bern and Geneva, Switzerland; MOST, Taiwan; TAEK, Turkey; STFC, United Kingdom; DOE and NSF, United States of America. In addition, individual groups and members have received support from BCKDF, CANARIE, CRC and Compute Canada, Canada; COST, ERC, ERDF, Horizon 2020, and Marie Sk lodowska-Curie Actions, European Union; Investissements d' Avenir Labex and Idex, ANR, France; DFG and AvH Foundation, Germany; Herakleitos, Thales and Aristeia programmes co-financed by EU-ESF and the Greek NSRF, Greece; BSF-NSF and GIF, Israel; CERCA Programme Generalitat de Catalunya, Spain; The Royal Society and Leverhulme Trust, United Kingdom.

The crucial computing support from all WLCG partners is acknowledged gratefully, in particular from CERN, the ATLAS Tier-1 facilities at TRIUMF (Canada), NDGF (Denmark, Norway, Sweden), CCIN2P3 (France), KIT/GridKA (Germany), INFN-CNAF (Italy), NLT1 (Netherlands), PIC (Spain), ASGC (Taiwan), RAL (UK) and BNL (USA), the Tier-2 facilities worldwide and large non-WLCG resource providers. Major contributors of computing resources are listed in Ref. [70].

Open Access This article is distributed under the terms of the Creative Commons Attribution 4.0 International License (http://creativecomm ons.org/licenses/by/4.0/), which permits unrestricted use, distribution, and reproduction in any medium, provided you give appropriate credit to the original author(s) and the source, provide a link to the Creative Commons license, and indicate if changes were made. Funded by $\mathrm{SCOAP}^{3}$.

\section{References}

1. J. Schechter, J.W.F. Valle, Neutrino masses in $S U(2) \otimes U(1)$ theories. Phys. Rev. D 22, 2227 (1980)

2. P. Fileviez Perez, T. Han, G-y Huang, T. Li, K. Wang, Neutrino masses and the CERN LHC: Testing the type II seesaw mechanisim. Phys. Rev. D 78, 015018 (2008). arXiv:0805.3536 [hep-ph]

3. A. Arhrib et al., The Higgs potential in the type II seesaw model. Phys. Rev. D 84, 095005 (2011) (We would like to thank Gilbert Moultaka for the implementation in CalcHEP of the doublet-tripletmodel and helpful discussions on the phenomenological aspects of the analysis). arXiv:1105.1925 [hep-ph]

4. S. Kanemura, M. Kikuchi, K. Yagyu, H. Yokoya, Bounds on the mass of doubly-charged Higgs bosons in the same-sign diboson decay scenario. Phys. Rev. D 90, 115018 (2014). arXiv:1407.6547 [hep-ph]

5. Z. Kang, J. Li, T. Li, Y. Liu, G.-Z. Ning, Light doubly charged Higgs boson via the $W W^{*}$ channel at LHC. Eur. Phys. J. C 75, 574 (2015). arXiv:1404.5207 [hep-ph]

6. OPAL collaboration, Search for doubly charged Higgs bosons with the OPAL detector at LEP. Phys. Lett. B 526, 221 (2002). arXiv:hep-ex/0111059

7. H1 Collaboration, Search for doubly-charged Higgs boson production at HERA. Phys. Lett. B 638, 432 (2006). arXiv:hep-ex/0604027

8. CDF Collaboration, Search for new physics in high $p_{T}$ like-sign Dilepton events at CDF II. Phys. Rev. Lett. 107, 181801 (2011). arXiv:1108.0101 [hep-ex]

9. ATLAS Collaboration, Search for doubly-charged Higgs bosons in like-sign dilepton final states at $\sqrt{s}=7 \mathrm{TeV}$ with the ATLAS detector. Eur. Phys. J. C 72, 2244 (2012). arXiv:1210.5070 [hep-ex]

10. CMS Collaboration, A search for a doubly-charged Higgs boson in pp collisions at $\sqrt{s}=7$ TeV. Eur. Phys. J. C 72, 2189 (2012). arXiv:1207.2666 [hep-ex] 
11. ATLAS Collaboration, Search for doubly charged Higgs boson production in multi-lepton final states with the ATLAS detector using proton-proton collisions at $\sqrt{s}=13 \mathrm{TeV}$. Eur. Phys. J. C 78, 199 (2018). arXiv:1710.09748 [hep-ex]

12. CMS Collaboration, Study of vector boson scattering and search for new physics in events with two same-sign leptons and two jets. Phys. Rev. Lett. 114, 051801 (2015). arXiv:1410.6315 [hep-ex]

13. CMS Collaboration, Observation of electroweak production of same-sign $\mathrm{W}$ boson pairs in the two jet and two same-sign lepton final state in proton-proton collisions at $\sqrt{\mathrm{s}}=13 \mathrm{TeV}$. Phys. Rev. Lett. 120, 081801 (2018). arXiv:1709.05822 [hep-ex]

14. H. Georgi, M. Machacek, Doubly charged Higgs bosons. Nucl. Phys. B 262, 463 (1985)

15. C. Englert, E. Re, M. Spannowsky, Triplet Higgs boson collider phenomenology after the LHC. Phys. Rev. D 87, 095014 (2013). arXiv:1302.6505 [hep-ph]

16. ATLAS Collaboration, Search for Higgs boson decays to beyondthe-Standard-Model light bosons in four-lepton events with the ATLAS detector at $\sqrt{s}=13 \mathrm{TeV}$ (2018). arXiv:1802.03388 [hepex]

17. ATLAS Collaboration, Search for electroweak production of supersymmetric particles in final states with two or three leptons at $\sqrt{s}=$ $13 \mathrm{TeV}$ with the ATLAS detector. (2018). arXiv:1803.02762 [hepex]

18. ATLAS Collaboration, Search for supersymmetry in final states with two same-sign or three leptons and jets using $36 \mathrm{fb}^{-1}$ of $\sqrt{s}=13 \mathrm{TeV}$ pp collision data with the ATLAS detector. JHEP 09, 084 (2017). arXiv:1706.03731 [hep-ex]

19. ATLAS Collaboration, The ATLAS experiment at the CERN large Hadron Collider. JINST 3, S08003 (2008)

20. ATLAS Collaboration, ATLAS Insertable B-Layer Technical Design Report, ATLAS-TDR-19 (2010). https://cds.cern.ch/ record/1291633. ATLAS Insertable B-Layer Technical Design Report Addendum, ATLAS-TDR-19-ADD-1 (2012). https://cds. cern.ch/record/1451888

21. ATLAS Collaboration, Performance of the ATLAS trigger system in 2015. Eur. Phys. J. C 77, 317 (2017). arXiv:1611.09661 [hep-ex]

22. ATLAS Collaboration, The ATLAS simulation infrastructure. Eur. Phys. J. C 70, 823 (2010). arXiv:1005.4568 [physics.ins-det]

23. S. Agostinelli et al., Geant4: a simulation toolkit. Nucl. Instrum. Meth. Phys. Res. A 506, 250 (2003)

24. ATLAS Collaboration, The simulation principle and performance of the ATLAS fast calorimeter simulation FastCaloSim. ATLPHYS-PUB-2010-013 (2010). https://cds.cern.ch/record/1300517

25. T. Sjöstrand, S. Mrenna, P. Z. Skands, PYTHIA 6.4 physics and manual. JHEP 05, 026 (2006). arXiv:hep-ph/0603175

26. T. Sjöstrand, S. Mrenna, P. Z. Skands, A brief introduction to PYTHIA 8.1. Comput. Phys. Commun. 178, 852 (2008). arXiv:0710.3820 [hep-ph]

27. ATLAS Collaboration, Monte Carlo Generators for the Production of a $\mathrm{W}$ or $Z / \gamma^{*}$ Boson in Association with Jets at ATLAS in Run 2. ATL-PHYS-PUB-2016-003. (2015). https://cds.cern.ch/record/ 2120133

28. A. Martin, W.J. Stirling, R.S. Thorne, G. Watt, Parton distributions for the LHC. Eur. Phys. J. C 63, 189 (2009). arXiv:0901.0002 [hep$\mathrm{ph}]$

29. A. Belyaev, N.D. Christensen, A. Pukhov, CalcHEP 3.4 for collider physics within and beyond the Standard Model. Comput. Phys. Commun. 184, 1729 (2013). arXiv:1207.6082 [hep-ph]

30. J. Pumplin, New generation of parton distributions with uncertainties from global QCD analysis. JHEP 07, 012 (2002). arXiv:hep-ph/0201195

31. P.M. Nadolsky, Implications of CTEQ global analysis for collider observables. Phys. Rev. D 78, 013004 (2008). arXiv:0802.0007 [hep-ph]
32. ATLAS Collaboration, ATLAS Pythia 8 tunes to $7 \mathrm{TeV}$ data. ATLPHYS-PUB-2014-021 (2014). https://cds.cern.ch/record/1966419

33. M. Muhlleitner, M. Spira, A Note on doubly charged Higgs pair production at hadron colliders. Phys. Rev. D 68, 117701 (2003). arXiv:hep-ph/0305288 [hep-ph]

34. D.J. Lange, The EvtGen particle decay simulation package. Nucl. Instrum. Meth. A 462, 152 (2001)

35. T. Gleisberg et al., Event generation with SHERPA 1.1. JHEP 02, 007 (2009). arXiv:0811.4622 [hep-ph]

36. H.-L. Lai, New parton distributions for collider physics. Phys. Rev. D 82, 074024 (2010). arXiv:1007.2241 [hep-ph]

37. J. Alwall, The automated computation of tree-level and next-toleading order differential cross sections, and their matching to parton shower simulations. JHEP 07, 079 (2014). arXiv:1405.0301 [hep-ph]

38. R.D. Ball, Parton distributions for the LHC Run II. JHEP 04, 040 (2015). arXiv:1410.8849 [hep-ph]

39. M. Bahr, Herwig++ physics and manual. Eur. Phys. J. C 58, 639 (2008). arXiv:0803.0883 [hep-ph]

40. M.H. Seymour, A. Siodmok, Constraining MPI models using $\sigma_{e f f}$ and recent Tevatron and LHC Underlying Event data. JHEP 10, 113 (2013). arXiv:1307.5015 [hep-ph]

41. P. Skands, Tuning Monte Carlo generators: The Perugia tunes. Phys. Rev. D 82, 074018 (2010). arXiv:1005.3457 [hep-ph]

42. E. Re, Single-top Wt-channel production matched with parton showers using the POWHEG method. Eur. Phys. J. C 71, 1547 (2011). arXiv:1009.2450 [hep-ph]

43. S. Alioli, P. Nason, C. Oleari, E. Re, NLO single-top production matched with shower in POWHEG: s- and t-channel contributions. JHEP 09, 111 (2009). arXiv:0907.4076 [hep-ph]

44. M. Grazzini, S. Kallweit, D. Rathlev, M. Wiesemann, $W^{ \pm} Z$ production at hadron colliders in NNLO QCD. Phys. Lett. B 761, 179 (2016). arXiv:1604.08576 [hep-ph]

45. F. Caola, K. Melnikov, R. Rontsch, L. Tancredi, QCD corrections to $\mathrm{ZZ}$ production in gluon fusion at the LHC. Phys. Rev. D 92, 094028 (2015). arXiv:1509.06734 [hep-ph]

46. M. Grazzini, S. Kallweit, D. Rathlev, ZZ production at the LHC: Fiducial cross sections and distributions in NNLO QCD. Phys. Lett. B 750, 407 (2015). arXiv:1507.06257 [hep-ph]

47. ATLAS Collaboration, Modelling of the $t \bar{t} H$ and $t \bar{t} V(V=W, Z)$ processes for $\sqrt{s}=13 \mathrm{TeV}$ ATLAS analyses, ATL-PHYS-PUB2016-005 (2016). https://cds.cern.ch/record/2120826

48. ATLAS Collaboration, Multi-Boson Simulation for $13 \mathrm{TeV}$ ATLAS Analyses, ATL-PHYS-PUB-2017-005 (2017). https://cds. cern.ch/record/2261933

49. ATLAS Collaboration, Validation of Monte Carlo event generators in the ATLAS Collaboration for LHC Run 2, ATL-PHYS-PUB2016-001. (2016). https://cds.cern.ch/record/2119984

50. ATLAS Collaboration, Vertex Reconstruction Performance of the ATLAS Detector at $\sqrt{s}=13 \mathrm{TeV}$, ATL-PHYS-PUB-2015-026 (2015). https://cds.cern.ch/record/2037717

51. ATLAS Collaboration, Electron efficiency measurements with the ATLAS detector using the 2015 LHC proton-proton collision data, ATLAS-CONF-2016-024 (2016). https://cds.cern.ch/record/ 2157687

52. ATLAS Collaboration, Muon reconstruction performance of the ATLAS detector in proton-proton collision data at $\sqrt{\mathrm{s}}=13 \mathrm{TeV}$. Eur. Phys. J. C 76, 292 (2016). arXiv:1603.05598 [hep-ex]

53. ATLAS Collaboration, Topological cell clustering in the ATLAS calorimeters and its performance in LHC Run 1. Eur. Phys. J. C 77, 490 (2017). arXiv:1603.02934 [hep-ex]

54. M. Cacciari, G.P. Salam, G. Soyez, The anti- $k_{t}$ jet clustering algorithm. JHEP 04, 063 (2008). arXiv:0802.1189 [hep-ph]

55. M. Cacciari, G.P. Salam, G. Soyez, FastJet user manual. Eur. Phys. J. C 72, 1896 (2012). arXiv:1111.6097 [hep-ph] 
56. ATLAS Collaboration, Performance of pile-up mitigation techniques for jets in pp collisions at $\sqrt{s}=8 \mathrm{TeV}$ using the ATLAS detector. Eur. Phys. J. C 76, 581 (2016). arXiv:1510.03823 [hep-ex]

57. ATLAS Collaboration, Performance of b-jet identification in the ATLAS experiment. JINST 11, P04008 (2016). arXiv:1512.01094 [hep-ex]

58. ATLAS Collaboration, Performance of missing transverse momentum reconstruction with the ATLAS detector using proton-proton collisions at $\sqrt{s}=13 \mathrm{TeV},(2018)$, arXiv:1802.08168 [hep-ex]

59. A. Hoecker et al., TMVA: Toolkit for Multivariate Data Analysis, PoS ACAT, 040 (2007). arXiv:physics/0703039

60. A. Buckley, LHAPDF6: parton density access in the LHC precision era. Eur. Phys. J. C 75, 132 (2015). arXiv:1412.7420 [hep-ph]

61. S. Gieseke, C. Röhr, A. Siódmok, Colour reconnections in Herwig++. Eur. Phys. J. C 72, 2225 (2012). arXiv:1206.0041 [hep-ph]

62. ATLAS Collaboration, Studies on top-quark Monte Carlo modelling with Sherpa and MG5_aMC@NLO, ATL-PHYS-PUB2017-007 (2017). https://cds.cern.ch/record/2261938

63. ATLAS Collaboration, Search for triboson $W^{ \pm} W^{ \pm} W^{\mp}$ production in pp collisions at $\sqrt{s}=8 \mathrm{TeV}$ with the ATLAS detector. Eur. Phys. J. C 77, 171 (2017). arXiv:1610.05088 [hep-ex]
64. ATLAS Collaboration, Measurement of the production crosssection of a single top quark in association with a $\mathrm{Z}$ boson in proton-proton collisions at $13 \mathrm{TeV}$ with the ATLAS detector. Phys. Lett. B 780, 557 (2018). arXiv:1710.03659 [hep-ex]

65. ATLAS Collaboration, Electron and photon energy calibration with the ATLAS detector using data collected in 2015 at $\sqrt{s}=$ $13 \mathrm{TeV}$, ATL-PHYS-PUB-2016-015, 2016. https://cds.cern.ch/ record/2203514

66. ATLAS Collaboration, Jet energy scale measurements and their systematic uncertainties in proton-proton collisions at $\sqrt{s}=$ $13 \mathrm{TeV}$ with the ATLAS detector, Phys. Rev. D 96 (2017) 072002, arXiv:1703.09665 [hep-ex]

67. ATLAS Collaboration, Luminosity determination in pp collisions at $\sqrt{s}=8 \mathrm{TeV}$ using the ATLAS detector at the LHC. Eur. Phys. J. C 76, 653 (2016). arXiv:1608.03953 [hep-ex]

68. G. Cowan, K. Cranmer, E. Gross, O. Vitells, Asymptotic formulae for likelihood-based tests of new physics. Eur. Phys. J. C 71, 1554 (2011). arXiv:1007.1727 [physics.data-an]

69. A.L. Read, Presentation of search results: the $C L_{s}$ technique. J. Phys. G 28, 2693 (2002)

70. ATLAS Collaboration, ATLAS Computing Acknowledgements, ATL-GEN-PUB-2016-002. https://cds.cern.ch/record/2202407

\section{ATLAS Collaboration}

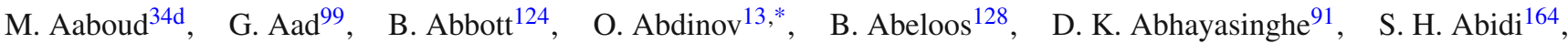
O. S. AbouZeid ${ }^{39}$, N. L. Abraham ${ }^{153}$, H. Abramowicz ${ }^{158}$, H. Abreu ${ }^{157}$, Y. Abulaiti ${ }^{6}$, B. S. Acharya ${ }^{64 a, 64 b, n}$, S. Adachi ${ }^{160}$, L. Adamczyk ${ }^{81 a}$, J. Adelman ${ }^{119}$, M. Adersberger ${ }^{112}$, A. Adiguzel ${ }^{12 c, a g}$, T. Adye ${ }^{141}$, A. A. Affolder ${ }^{143}$, Y. Afik ${ }^{157}$, C. Agheorghiesei ${ }^{27 c}$, J. A. Aguilar-Saavedra ${ }^{136 a, 136 f}$, F. Ahmadov ${ }^{77, a e}$, G. Aielli ${ }^{71 a, 71 b}$, S. Akatsuka ${ }^{83}$, T. P. A. Åkesson ${ }^{94}$,

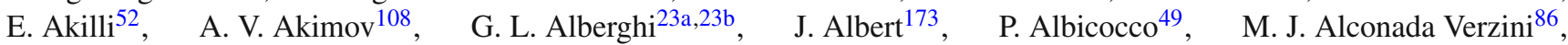
S. Alderweireldt ${ }^{117}$, M. Aleksa ${ }^{35}$, I. N. Aleksandrov ${ }^{77}$, C. Alexa ${ }^{27 b}$, T. Alexopoulos ${ }^{10}$, M. Alhroob ${ }^{124}$, B. Ali ${ }^{138}$, G. Alimonti6 ${ }^{66 a}$, J. Alison ${ }^{36}$, S. P. Alkire ${ }^{145}$, C. Allaire ${ }^{128}$, B. M. M. Allbrooke ${ }^{153}$, B. W. Allen ${ }^{127}$, P. P. Allport ${ }^{21}$, A. Aloisio ${ }^{67 a, 67 b}$, A. Alonso ${ }^{39}$, F. Alonso ${ }^{86}$, C. Alpigiani ${ }^{145}$, A. A. Alshehri ${ }^{55}$, M. I. Alstaty ${ }^{99}$, B. Alvarez Gonzalez ${ }^{35}$, D. Álvarez Piqueras ${ }^{171}$, M. G. Alviggi67a,67b， B. T. Amadio ${ }^{18}$, Y. Amaral Coutinho ${ }^{78 b}$, L. Ambroz ${ }^{131}$, C. Amelung ${ }^{26}$, D. Amidei ${ }^{103}$, S. P. Amor Dos Santos ${ }^{136 a, 136 c}$, S. Amoroso ${ }^{44}$, C. S. Amrouche ${ }^{52}$, C. Anastopoulos ${ }^{146}$, L. S. Ancu ${ }^{52}$, N. Andari ${ }^{21}$ ， T. Andeen ${ }^{11}$ ， C. F. Anders ${ }^{59 b}$ ， J. K. Anders $^{20}$ ， K. J. Anderson ${ }^{36}$ ， A. Andreazza ${ }^{66 a, 66 b}$, V. Andrei ${ }^{59 a}$, C. R. Anelli1 ${ }^{173}$, S. Angelidakis ${ }^{37}$, I. Angelozzi ${ }^{118}$, A. Angerami ${ }^{38}$, A. V. Anisenkov ${ }^{120 a, 120 b}$, A. Annovi ${ }^{69 a}$, C. Antel ${ }^{59 a}$, M. T. Anthony ${ }^{146}$, M. Antonellii" ${ }^{49}$ D. J. A. Antrim ${ }^{168}$, F. Anulli ${ }^{70 a}$, M. Aoki ${ }^{79}$, J. A. Aparisi Pozo ${ }^{171}$, L. Aperio Bella ${ }^{35}$, $\begin{array}{lll}\text { G. Arabidze } & 104 & \text { J. P. Araque } \\ & 136 \mathrm{a} & \text { V. Araujo Ferraz }\end{array}$ F. A. $\operatorname{Arduh}^{86}$, J-F. Arguin ${ }^{107}$, S. Argyropoulos ${ }^{75}$, A. J. Armbruster ${ }^{35}$, L. J. Armitage ${ }^{90}$, A Armstrong ${ }^{168}$, O. Arnaez ${ }^{164}$, H. Arnold ${ }^{118}$, M. Arratia ${ }^{31}$, O. Arslan ${ }^{24}$, A. Artamonov ${ }^{109, *}$, G. Artoni ${ }^{131}, \quad$ S. Artz ${ }^{97}, \quad$ S. Asai ${ }^{160}, \quad$ N. Asbah ${ }^{44}$, A. Ashkenazi ${ }^{158}$, E. M. Asimakopoulou ${ }^{169}$, L. Asquith ${ }^{153}$, K. Assamagan ${ }^{29}$, R. Astalos ${ }^{28 a}$, R. J. Atkin ${ }^{32 a}$, M. Atkinson ${ }^{170}$, N. B. Atlay ${ }^{148}, \quad$ K. Augsten ${ }^{138}, \quad$ G. Avolio ${ }^{35}$, R. Avramidou${ }^{58 a}$, M. K. Ayoub ${ }^{15 a}$ ， G. Azuelos ${ }^{107, a s, ~ A . ~ E . ~ B a a s ~}{ }^{59 a}$, M. J. Baca ${ }^{21}$, H. Bachacou ${ }^{142}$, K. Bachas ${ }^{65 a, 65 b}$, M. Backes ${ }^{131}$, P. Bagnaia ${ }^{70 a, 70 b}$, M. Bahmani ${ }^{82}$, H. Bahrasemani ${ }^{149}$,

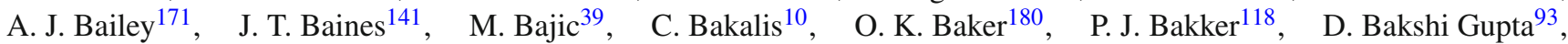

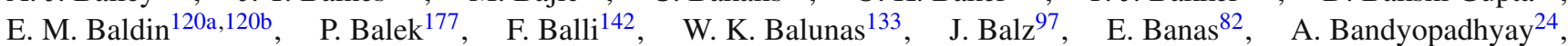
S. Banerjee ${ }^{178, j}$, A. A. E. Bannoura ${ }^{179}$, L. Barak ${ }^{158}$, W. M. Barbe ${ }^{37}$, E. L. Barberio ${ }^{102}$, D. Barberis ${ }^{53 a, 53 b}$, M. Barbero ${ }^{99}$, T. Barillari ${ }^{113}$, M-S. Barisits ${ }^{35}$, J. Barkeloo ${ }^{127}$, T. Barklow ${ }^{150}$, N. Barlow ${ }^{31}$, R. Barnea ${ }^{157}$, S. L. Barnes ${ }^{58 c}$, B. M. Barnett ${ }^{141}$, R. M. Barnett ${ }^{18}$ ， Z. Barnovska-Blenessy ${ }^{58 a}$ ， A. Baroncelli ${ }^{72 a}$ ， G. Barone ${ }^{26}$ ， A. J. Barr ${ }^{131}$ ， L. Barranco Navarro ${ }^{171}$, F. Barreiro ${ }^{96}$, J. Barreiro Guimarães da Costa ${ }^{15 a}$, R. Bartoldus ${ }^{150}$, A. E. Barton ${ }^{87}, \quad$ P. Bartos ${ }^{28 a}$, A. Basalaev ${ }^{134}$, A. Bassalat ${ }^{128}$, R. L. Bates ${ }^{55}$, S. J. Batista ${ }^{164}$, S. Batlamous ${ }^{34 \mathrm{e}}$, J. R. Batley ${ }^{31}$, M. Battaglia ${ }^{143}$, M. Bauce ${ }^{70 a}, 70 \mathrm{~b}$, F. Bauer ${ }^{142}$, K. T. Bauer ${ }^{168}$ ， H. S. Bawa ${ }^{150,1}$ ， J. B. Beacham ${ }^{122}$ ， M. D. Beattie ${ }^{87}$ ， T. Beau ${ }^{132}$, P. H. Beauchemin ${ }^{167}$ ， P. Bechtle ${ }^{24}$,

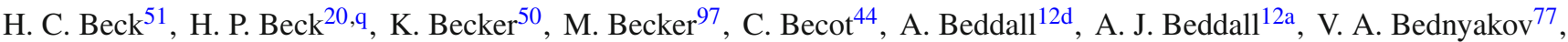
M. Bedognetti ${ }^{118}$, C. P. Bee ${ }^{152}$, T. A. Beermann ${ }^{35}$, M. Begalli ${ }^{78 b}$, M. Begel ${ }^{29}$, A. Behera ${ }^{152}$, J. K. Behr ${ }^{44}$, A. S. Bell ${ }^{92}$, G. Bella ${ }^{158}$, L. Bellagamba ${ }^{23 b}$, A. Bellerive ${ }^{33}, \quad$ M. Bellomo ${ }^{157}$ ， P. Bellos ${ }^{9}, \quad$ K. Belotskiy ${ }^{110}$, N. L. Belyaev ${ }^{110}$, O. Benary ${ }^{158, *}$, D. Benchekroun ${ }^{34 a}$, M. Bender ${ }^{112}$, N. Benekos ${ }^{10}$, Y. Benhammou ${ }^{158}$, E. Benhar Noccioli ${ }^{180}$, J. Benitez ${ }^{75}$, 
D. P. Benjamin ${ }^{47}$ ， M. Benoit ${ }^{52}$ ， J. R. Bensinger ${ }^{26}, \quad$ S. Bentvelsen ${ }^{118}, \quad$ L. Beresford ${ }^{131}, \quad$ M. Beretta ${ }^{49}$ ， D. Berge ${ }^{44}$, E. Bergeaas Kuutmann ${ }^{169}$, N. Berger ${ }^{5}$, L. J. Bergsten ${ }^{26}$, J. Beringer ${ }^{18}$, S. Berlendis ${ }^{7}$, N. R. Bernard ${ }^{100}$, G. Bernardi ${ }^{132}$, C. Bernius ${ }^{150}$, F. U. Bernlochner ${ }^{24}$, T. Berry ${ }^{91}$, P. Berta ${ }^{97}$, C. Bertella ${ }^{15 a}$, G. Bertoli ${ }^{43 a, 43 b}$, I. A. Bertram ${ }^{87}$, G. J. Besjes ${ }^{39}$, O. Bessidskaia Bylund ${ }^{43 a}, 43 b$, M. Bessner ${ }^{44}$, N. Besson ${ }^{142}$, A. Bethani ${ }^{98}$, S. Bethke ${ }^{113}$, A. Betti ${ }^{24}$, A. J. Bevan ${ }^{90}$, J. Beyer ${ }^{113}$, R. M. Bianchi ${ }^{135}$, O. Biebel ${ }^{112}$, D. Biedermann ${ }^{19}$ ， R. Bielski ${ }^{98}$ ，K. Bierwagen ${ }^{97}$ ， N. V. Biesuz ${ }^{69 a, 69 b}$, M. Biglietti ${ }^{72 a}$, T. R. V. Billoud ${ }^{107}$, M. Bindi ${ }^{51}$, A. Bingul ${ }^{12 d}$, C. Bini ${ }^{70 a, 70 b}$, S. Biondi ${ }^{23 a, 23 b}$, M. Birman ${ }^{177}$, T. Bisanz ${ }^{51}$, J. P. Biswal ${ }^{158}$, C. Bittrich ${ }^{46}$, D. M. Bjergaard ${ }^{47}$, J. E. Black ${ }^{150}$, K. M. Black ${ }^{25}$, T. Blazek ${ }^{28 a}$, I. Bloch ${ }^{44}$, C. Blocker ${ }^{26}$, A. Blue ${ }^{55}$, U. Blumenschein ${ }^{90}$ ，Dr. Blunier ${ }^{144 a}$ ， G. J. Bobbink ${ }^{118}$ ， V. S. Bobrovnikov ${ }^{120 a, 120 b}$, S. S. Bocchetta ${ }^{94}$, A. Bocci ${ }^{47}$, D. Boerner ${ }^{179}$, D. Bogavac ${ }^{112}$, A. G. Bogdanchikov ${ }^{120 a, 120 b}$, C. Bohm ${ }^{43 a}$, V. Boisvert ${ }^{91}$, P. Bokan ${ }^{169,51}$, T. Bold ${ }^{81 a}$, A. S. Boldyrev" ${ }^{111}$ A. E. Bolz ${ }^{59 b}, \quad$ M. Bomben ${ }^{132}, \quad$ M. Bona ${ }^{90}, \quad$ J. S. Bonilla ${ }^{127}, \quad$ M. Boonekamp ${ }^{142}$, A. Borisov ${ }^{140}$, G. Borissov ${ }^{87}$, J. Bortfeldt ${ }^{35}$, D. Bortoletto ${ }^{131}$, V. Bortolotto ${ }^{61 b, 61 c, 71 a, 71 b}$, D. Boscherini2 ${ }^{23 b}$, M. Bosman ${ }^{14}$, J. D. Bossio Sola ${ }^{30}$ ， K. Bouaouda ${ }^{34 a}$ ， J. Boudreau ${ }^{135}$, E. V. Bouhova-Thacker ${ }^{87}$ ，D. Boumediene ${ }^{37}$ ， C. Bourdarios ${ }^{128}$, S. K. Boutle ${ }^{55}$, A. Boveia ${ }^{122}$ ， J. Boyd ${ }^{35}$ ， I. R. Boyko ${ }^{77}$, A. J. Bozson ${ }^{91}$ ， J. Bracinik ${ }^{21}$ ， N. Brahimi ${ }^{99}$, A. Brandt ${ }^{8}$, G. Brandt ${ }^{179}$ ， O. Brandt ${ }^{59 a}$ ， F. Braren ${ }^{44}$, U. Bratzler ${ }^{161}$ ， B. Brau ${ }^{100}$ ， J. E. Brau ${ }^{127}$ ， W. D. Breaden Madden ${ }^{55}$, K. Brendlinger ${ }^{44}$, A. J. Brennan ${ }^{102}$, L. Brenner ${ }^{44}$, R. Brenner ${ }^{169}$, S. Bressler ${ }^{177}$, B. Brickwedde ${ }^{97}$, D. L. Briglinn ${ }^{21}$, D. Britton ${ }^{55}$, D. Britzger ${ }^{59 b}$, I. Brock ${ }^{24}$, R. Brock ${ }^{104}$, G. Brooijmans ${ }^{38}$, T. Brooks ${ }^{91}$, W. K. Brooks ${ }^{144 b}$, E. Brost ${ }^{119}$, J. H Broughton ${ }^{21}$, P. A. Bruckman de Renstrom ${ }^{82}$, D. Bruncko ${ }^{28 b}$, A. Bruni ${ }^{23 b}$, G. Bruni ${ }^{23 b}$, L. S. Bruni ${ }^{118}$, S. Bruno ${ }^{71 a, 71 b}$, B.H. Brunt ${ }^{31}$, M. Bruschi ${ }^{23 \mathrm{~b}}$, N. Bruscino ${ }^{135}$, P. Bryant ${ }^{36}$, L. Bryngemark ${ }^{44}$, T. Buanes ${ }^{17}$, Q. Buat ${ }^{35}$, P. Buchholz ${ }^{148}$, A. G. Buckley ${ }^{55}$, I. A. Budagov ${ }^{77}$, F. Buehrer ${ }^{50}$, M. K. Bugge ${ }^{130}$, O. Bulekov ${ }^{110}$, D. Bullock ${ }^{8}$, T. J. Burch ${ }^{119}$, S. Burdinn ${ }^{88}$,

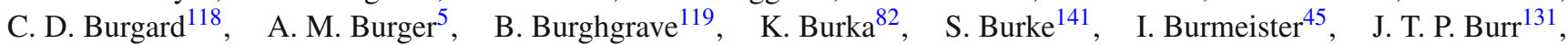
D. Büscher ${ }^{50}$, V. Büscher ${ }^{97}$, E. Buschmann ${ }^{51}$, P. Bussey ${ }^{55}$, J. M. Butler ${ }^{25}$, C. M. Buttar ${ }^{55}$, J. M. Butterworth ${ }^{92}$, P. Butti ${ }^{35}$, W. Buttinger ${ }^{35}$, A. Buzatu ${ }^{155}$, A. R. Buzykaev ${ }^{120 a, 120 b}$, G. Cabras ${ }^{23 a, 23 b}$, S. Cabrera Urbán ${ }^{171}$, D. Caforio ${ }^{138}$, H. Cai ${ }^{170}$, V. M. M. Cairo ${ }^{2}$, O. Cakir $^{4 a}$, N. Calace ${ }^{52}$, P. Calafiura ${ }^{18}$, A. Calandri ${ }^{99}$, G. Calderini ${ }^{132}$, P. Calfayan ${ }^{63}$, G. Callea ${ }^{40 a, 40 b}$, L. P. Caloba ${ }^{78 b}$, S. Calvente Lopez ${ }^{96}$, D. Calvet $^{37}$, S. Calvet ${ }^{37}$, T. P. Calvet ${ }^{152}$, M. Calvetti ${ }^{69 a, 69 b}$, R. Camacho Toro ${ }^{132}$, S. Camarda ${ }^{35}$, P. Camarri ${ }^{71 a}, 71 b$, D. Cameron ${ }^{130}$, R. Caminal Armadans ${ }^{100}$, C. Camincher ${ }^{35}$, S. Campana ${ }^{35}$, M. Campanelli92, A. Camplani ${ }^{39}$, A. Campoverde ${ }^{148}$, V. Canale ${ }^{67 \mathrm{a}, 67 \mathrm{~b}}$, M. Cano Bret ${ }^{58 \mathrm{c}}$, J. Cantero ${ }^{125}$, T. Cao ${ }^{158}$, Y. Cao ${ }^{170}$, M. D. M. Capeans Garrido ${ }^{35}$, I. Caprini ${ }^{27 b}$, M. Caprini ${ }^{27 b}$, M. Capua ${ }^{40 a, 40 b}$, R. M. Carbone ${ }^{38}$, R. Cardarelli ${ }^{71 a}$, F. C. Cardillo ${ }^{50}$, I. Carli ${ }^{139}$, T. Carli ${ }^{35}$, G. Carlino ${ }^{67 a}$, B. T. Carlson ${ }^{135}$, L. Carminati66a,66b , R. M. D. Carney ${ }^{43 a, 43 b}$, S. Caron ${ }^{117}$, E. Carquin ${ }^{144 b}$, S. Carrá66a,66b, G. D. Carrillo-Montoya ${ }^{35}$, D. Casadei ${ }^{32 b}$, M. P. Casado ${ }^{14, f}$, A. F. Casha ${ }^{164}$,

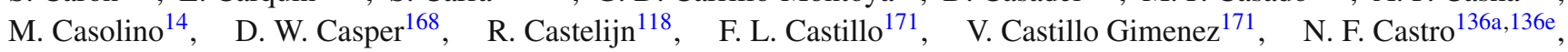

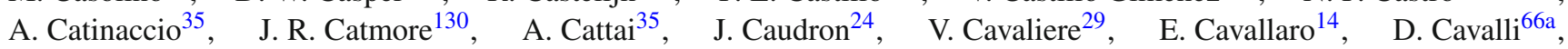
M. Cavalli-Sforza ${ }^{14}$, V. Cavasinni69a,69b, E. Celebi ${ }^{12 b}$, F. Ceradini ${ }^{72 a, 72 b}$, L. Cerda Alberich ${ }^{171}$, A. S. Cerqueira ${ }^{78 a}$, A. Cerri ${ }^{153}$, L. Cerrito ${ }^{71 a}, 71 b$, F. Cerutti ${ }^{18}$, A. Cervelli ${ }^{23 a, 23 b}$, S. A. Cetin ${ }^{12 b}$, A. Chafaq ${ }^{34 a}$, D Chakraborty ${ }^{119}$, S. K. Chan ${ }^{57}$, W. S. Chan ${ }^{118}$, Y. L. Chan ${ }^{61 a}$, J. D. Chapman ${ }^{31}$, D. G. Charlton ${ }^{21}$, C. C. Chau ${ }^{33}$, C. A. Chavez Barajas ${ }^{153}$, S. Che ${ }^{122}$,

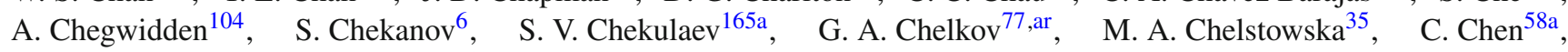

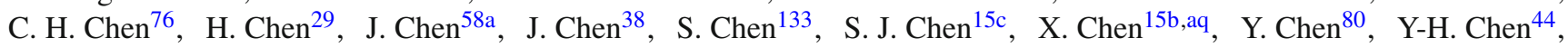
H. C. Cheng ${ }^{103}$, H. J. Cheng ${ }^{15 d}$, A. Cheplakov ${ }^{77}, \quad$ E. Cheremushkina ${ }^{140}, \quad$ R. Cherkaoui El Moursli3 ${ }^{34 e, ~ E . ~ C h e u ~}{ }^{7}$, K. Cheung ${ }^{62}$, L. Chevalier ${ }^{142}$, V. Chiarella ${ }^{49}$, G. Chiarellii ${ }^{69}$, G. Chiodini ${ }^{65 a}$, A. S. Chisholm ${ }^{35}$, A. Chitan ${ }^{27 b}$, I. Chiu ${ }^{160}$, Y. H. Chiu ${ }^{173}$, M. V. Chizhov ${ }^{77}$, K. Choi ${ }^{63}$, A. R. Chomont ${ }^{128}$, S. Chouridou ${ }^{159}$, Y. S. Chow ${ }^{118}$, V. Christodoulou ${ }^{92}$,

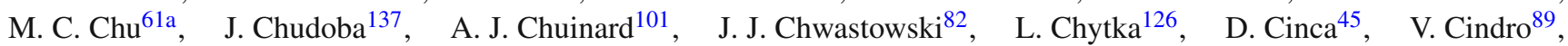
I. A. Cioară ${ }^{24}$, A. Ciocio ${ }^{18}$, F. Cirotto ${ }^{67 a, 67 b}$, Z. H. Citron ${ }^{177}$, M. Citterio ${ }^{66 a}$, A. Clark ${ }^{52}$, M. R. Clark ${ }^{38}$, P. J. Clark ${ }^{48}$, C. Clement ${ }^{43 a}, 43 b$, Y. Coadou ${ }^{99}$, M. Cobal ${ }^{64 a, 64 c}$, A. Coccaro ${ }^{53 a, 53 b}$, J. Cochran ${ }^{76}$, A. E. C. Coimbra ${ }^{177}$, L. Colasurdo ${ }^{117}$, B. Cole $^{38}$, A. P. Colijn ${ }^{118}$, J. Collot ${ }^{56}$, P. Conde Muiño ${ }^{136 a, 136 b}$, E. Coniavitis ${ }^{50}$, S. H. Connell ${ }^{32 b}$, I. A. Connelly ${ }^{98}$, S. Constantinescu ${ }^{27 b}$, F. Conventi6 ${ }^{67 a}$,at, A. M. Cooper-Sarkar ${ }^{131}$, F. Cormier ${ }^{172}$, K. J. R. Cormier ${ }^{164}$, M. Corradi ${ }^{70 a, 70 b}$, E. E. Corrigan ${ }^{94}$, F. Corriveau ${ }^{101, a c}$, A. Cortes-Gonzalez ${ }^{35}$, M. J. Costa ${ }^{171}$, D. Costanzo ${ }^{146}$, G. Cottin ${ }^{31}$, G. Cowan ${ }^{91}$, B. E. $\mathrm{Cox}^{98}$, J. Crane ${ }^{98}$, K. Cranmer ${ }^{121}$, S. J. Crawley ${ }^{55}$, R. A. Creager ${ }^{133}$, G. Cree ${ }^{33}$, S. Crépé-Renaudin ${ }^{56}$, F. Crescioli ${ }^{132}$, M. Cristinziani ${ }^{24}$, V. Croft ${ }^{121}$, G. Crosetti ${ }^{40 a, 40 b}$, A. Cueto ${ }^{96}$, T. Cuhadar Donszelmann ${ }^{146}$, A. R. Cukierman ${ }^{150}$, J. Cúth ${ }^{97}$, S. Czekierda ${ }^{82}$, P. Czodrowski ${ }^{35}$, M. J. Da Cunha Sargedas De Sousa ${ }^{58 b, 136 b}$, C. Da Via ${ }^{98}$, W. Dabrowski ${ }^{81 a}$, T. Dado ${ }^{28 a, x}$, S. Dahbi ${ }^{34 \mathrm{e}}$, T. Dai ${ }^{103}$, F. Dallaire ${ }^{107}$, C. Dallapiccola ${ }^{100}$, M. Dam ${ }^{39}$ ， G. D'amenn ${ }^{23 a, 23 b}$, J. Dampp7, J. R. Dandoy ${ }^{133}$, M. F. Daneri ${ }^{30}$, N. P. Dang ${ }^{178, j}$, N. D Dann ${ }^{98}$, M. Danninger ${ }^{172}$, V. Dao ${ }^{35}$ ， G. Darbo ${ }^{53 b}$ ， S. Darmora ${ }^{8}$, O. Dartsi ${ }^{5}$, A. Dattagupta ${ }^{127}$ ， T. Daubney ${ }^{44}$ ，S. D’Auria ${ }^{55}$ ，W. Davey ${ }^{24}$ ，C. David ${ }^{44}$ ，T. Davidek ${ }^{139}$ ，D. R. Davis ${ }^{47}$ ， E. Dawe ${ }^{102}$, I. Dawson ${ }^{146}$, K. De ${ }^{8}$, R. De Asmundis ${ }^{67 a}$, A. De Benedetti ${ }^{124}$, M. De Beurs ${ }^{118}$, S. De Castro ${ }^{23 a, 23 b}$, S. De Cecco ${ }^{70 a, 70 b}$, N. De Groot ${ }^{117}$, P. de Jong ${ }^{118}$, H. De la Torre ${ }^{104}$, F. De Lorenzi ${ }^{76}$, A. De Maria ${ }^{51, \mathrm{~s}}$, D. De Pedis ${ }^{70 a}$, A. De Salvo ${ }^{70 a}$, 
U. De Sanctis ${ }^{71 a, 71 b}$, A. De Santo ${ }^{153}$ ， K. De Vasconcelos Corga ${ }^{99}$ ， J. B. De Vivie De Regie ${ }^{128}$ ， C. Debenedetti ${ }^{143}$, D. V. Dedovich ${ }^{77}$, N. Dehghanian ${ }^{3}$, M. Del Gaudio ${ }^{40 a, 40 b}$, J. Del Peso ${ }^{96}$, Y. Delabat Diaz ${ }^{44}$, D. Delgove ${ }^{128}$, F. Deliot ${ }^{142}$, C. M. Delitzsch ${ }^{7}$, M. Della Pietra ${ }^{67 a, 67 b}$, D. Della Volpe ${ }^{52}$, A. Dell'Acqua ${ }^{35}$, L. Dell'Asta ${ }^{25}$, M. Delmastro ${ }^{5}$, C. Delporte ${ }^{128}$, P. A. Delsart ${ }^{56}$, D. A. DeMarco ${ }^{164}$, S. Demers ${ }^{180}$, M. Demichev ${ }^{77}$, S. P. Denisov ${ }^{140}$, D. Denysiuk ${ }^{118}$, L. D'Eramo ${ }^{132}$, D. Derendarz ${ }^{82}$, J. E. Derkaoui ${ }^{34 d}$, F. Derue ${ }^{132}$, P. Dervan ${ }^{88}$, K. $\operatorname{Desch}^{24}$, C. Deterre ${ }^{44}$, K. Dette ${ }^{164}$, M. R. Devesa $^{30}$, P. O. Deviveiros ${ }^{35}$, A. Dewhurst ${ }^{141}$, S. Dhaliwal ${ }^{26}$, F. A. Di Bello ${ }^{52}$, A. Di Ciaccio ${ }^{71 a}, 71 b$, L. Di Ciaccio ${ }^{5}$, W. K. Di Clemente ${ }^{133}$, C. Di Donato67a,67b, A. Di Girolamo ${ }^{35}$ ，B. Di Micco ${ }^{72 a, 72 b}$ ，R. Di Nardo ${ }^{100}$ ，K. F. Di Petrillo ${ }^{57}$, A. Di Simone ${ }^{50}$, R. Di Sipio ${ }^{164}$, D. Di Valentino ${ }^{33}$, C. Diaconu ${ }^{99}$, M. Diamond ${ }^{164}$, F. A. Dias ${ }^{39}$, T. Dias Do Vale ${ }^{136 a}$, M. A. Diaz ${ }^{144 a}$, J. Dickinson ${ }^{18}$, E. B. Diehl ${ }^{103}$, J. Dietrich ${ }^{19}$ ， S. Díez Cornell ${ }^{44}$, A. Dimitrievska ${ }^{18}$, J. Dingfelder ${ }^{24}$, F. Dittus ${ }^{35}$ ， F. Djama ${ }^{99}$ ， T. Djobava ${ }^{156 b}$ ， J. I. Djuvsland ${ }^{59 a} ，$ M. A. B. Do Vale ${ }^{78 c} ，$ M. Dobre ${ }^{27 b} ，$ D. Dodsworth ${ }^{26}$, C. Doglioni ${ }^{94}$, J. Dolejsi ${ }^{139}$, Z. Dolezal ${ }^{139}$, M. Donadelli7 ${ }^{78 d}$, J. Donini ${ }^{37}$, A. D'onofrio ${ }^{90}$, M. D’Onofrio ${ }^{88}$, J. Dopke ${ }^{141}$, A. Doria ${ }^{67 a}$, M. T. Dova ${ }^{86}$, A. T. Doyle ${ }^{55}$, E. Drechsler ${ }^{51}$, E. Dreyer ${ }^{149}$, T. Dreyer ${ }^{51}$, Y. Du ${ }^{58 b}$, J. Duarte-Campderros ${ }^{158}$, F. Dubinin ${ }^{108}$, M. Dubovsky ${ }^{28 a}$, A. Dubreuil ${ }^{52}$, E. Duchovni1 ${ }^{177}$, G. Duckeck ${ }^{112}$, A. Ducourthial ${ }^{132}$, O. A. Ducu ${ }^{107, w}$, D. Duda ${ }^{113}$, A. Dudarev ${ }^{35}$, A. C. Dudder ${ }^{97}$, E. M. Duffield ${ }^{18}$, L. Duflot ${ }^{128}$, M. Dührssen ${ }^{35}$, C. Dülsen ${ }^{179}$, M. Dumancic ${ }^{177}$, A. E. Dumitriu ${ }^{27 b, d}$, A. K. Duncan ${ }^{55}$, M. Dunford ${ }^{59 a}$, A. Duperrin ${ }^{99}$, H. Duran Yildiz ${ }^{4 a}$, M. Düren ${ }^{54}$, A. Durglishvili ${ }^{156 b}$, D. Duschinger ${ }^{46}$, B. Dutta ${ }^{44}$, D. Duvnjak ${ }^{1}$, M. Dyndal ${ }^{44}$, S. Dysch ${ }^{98}$, B. S. Dziedzic ${ }^{82}$, C. Eckardt ${ }^{44}$, K. M. Ecker ${ }^{113}$, R. C. Edgar $^{103}$, T. Eifert ${ }^{35}$, G. Eigen ${ }^{17}$, K. Einsweiler ${ }^{18}$, T. Ekelof ${ }^{169}$, M. El Kacimi ${ }^{34 c}$, R. El Kosseifi ${ }^{99}$, V. Ellajosyula ${ }^{99}$, M. Ellert ${ }^{169}$, F. Ellinghaus ${ }^{179}$, A. A. Elliot ${ }^{90}$, N. Ellis $^{35}$, J. Elmsheuser ${ }^{29}$, M. Elsing ${ }^{35}$, D. Emeliyanov ${ }^{141}$, Y. Enari ${ }^{160}$,

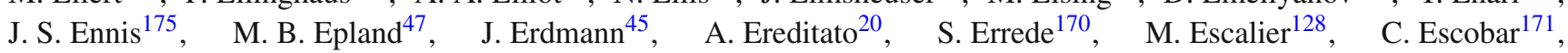
O. Estrada Pastor ${ }^{171}$, A. I. Etienvre ${ }^{142}$, E. Etzion ${ }^{158}$, H. Evans ${ }^{63}$, A. Ezhilov ${ }^{134}$, M. Ezzi ${ }^{34 e}$, F. Fabbri ${ }^{55}$, L. Fabbri ${ }^{23 a}$,23b, V. Fabiani ${ }^{117}$ ， G. Facini ${ }^{92}$ ， R. M. Faisca Rodrigues Pereira ${ }^{136 a}$ ， R. M. Fakhrutdinov ${ }^{140}$ ， S. Falciano ${ }^{70 a, ~ P . ~ J . ~ F a l k e ~}{ }^{5}$, S. Falke ${ }^{5}$, J. Faltova ${ }^{139}$, Y. Fang ${ }^{15 a}$, M. Fanti66a,66b, A. Farbin ${ }^{8}$, A. Farilla ${ }^{72 a}$, E. M. Farina ${ }^{68 a, 68 b}$, T. Farooque ${ }^{104}$, S. Farrel1 ${ }^{18}$, S. M. Farrington ${ }^{175}$, P. Farthouat ${ }^{35}$, F. Fassi ${ }^{34 \mathrm{e}}$, P. Fassnacht ${ }^{35}$, D. Fassouliotis ${ }^{9}$, M. Faucci Giannelli ${ }^{48}$, A. Favareto $53 \mathrm{a}, 53 \mathrm{~b}$, W. J. Fawcett ${ }^{52}$, L. Fayard ${ }^{128}, \quad$ O. L. Fedin ${ }^{134, \mathrm{o}}$, W. Fedorko ${ }^{172}, \quad$ M. Feickert ${ }^{41}, \quad$ S. Feigl ${ }^{130}$, L. Feligioni ${ }^{99}$, C. Feng ${ }^{58 b}$, E. J. Feng ${ }^{35}$, M. Feng ${ }^{47}$, M. J. Fenton ${ }^{55}$, A. B. Fenyuk ${ }^{140}$, L. Feremenga ${ }^{8}$, J. Ferrando ${ }^{44}$, A. Ferrari ${ }^{169}$, P. Ferrari ${ }^{118}$, R. Ferrari ${ }^{68 a}$, D. E. Ferreira de Lima $^{59 b}$, A. Ferrer ${ }^{171}$, D. Ferrere ${ }^{52}$, C. Ferretti ${ }^{103}$, F. Fiedler ${ }^{97}$, A. Filipčič ${ }^{89}$, F. Filthaut ${ }^{117}$, K. D. Finelli ${ }^{25}$, M. C. N. Fiolhais ${ }^{136 a, 136 c, a}$, L. Fiorini ${ }^{171}$, C. Fischer ${ }^{14}$, W. C. Fisher ${ }^{104}$, N. Flaschel ${ }^{44}$, I. Fleck ${ }^{148}$, P. Fleischmann ${ }^{103}$, R. R. M. Fletcher ${ }^{133}$, T. Flick ${ }^{179}$, B. M. Flierl ${ }^{112}$, L. M. Flores ${ }^{133}$, L. R. Flores Castillo ${ }^{61 a}$, N. Fomin ${ }^{17}$, G. T. Forcolin ${ }^{98}$, A. Formica ${ }^{142}$, F. A. Förster ${ }^{14}$, A. C. Forti ${ }^{98}$, A. G. Foster ${ }^{21}$, D. Fournier ${ }^{128}$, H. Fox ${ }^{87}$, S. Fracchia ${ }^{146}$, P. Francavilla ${ }^{69 a, 69 b}$, M. Franchini ${ }^{23 a, 23 b}$, S. Franchino ${ }^{59 a}$, D. Francis ${ }^{35}$,

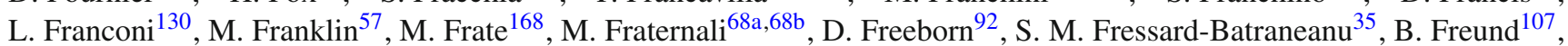
W. S. Freund ${ }^{78 b}$, D. Froidevaux ${ }^{35}$, J. A. Frost ${ }^{131}$, C. Fukunaga ${ }^{161}$, E. Fullana Torregrosa ${ }^{171}$, T. Fusayasu ${ }^{114}$, J. Fuster ${ }^{171}$, O. Gabizon ${ }^{157}$, A. Gabrielli23a,23b, A. Gabrielli ${ }^{18}$, G. P. Gach ${ }^{81 a}$, S. Gadatsch ${ }^{52}$, P. Gadow ${ }^{113}$, G. Gagliardi ${ }^{53 a, 53 b}$, L. G. Gagnon ${ }^{107}$, C. Galea ${ }^{27 b}$, B. Galhardo ${ }^{136 a, 136 c}$, E. J. Gallas ${ }^{131}$, B. J. Gallop ${ }^{141}$, P. Gallus ${ }^{138}$, G. Galster ${ }^{39}$, R. Gamboa Goni ${ }^{90}$, K. K. Gan ${ }^{122}$, S. Ganguly ${ }^{177}$, J. Gao ${ }^{58 a}$, Y. Gao ${ }^{88}$, Y. S. Gao ${ }^{150,1}$, C. García ${ }^{171}$, J. E. García Navarro ${ }^{171}$, J. A. García Pascual ${ }^{15 a}$, M. Garcia-Sciveres ${ }^{18}, \quad$ R. W. Gardner ${ }^{36}, \quad$ N. Garelli ${ }^{150}, \quad$ V. Garonne ${ }^{130}, \quad$ K. Gasnikova ${ }^{44}$, A. Gaudiello53a,53b, G. Gaudio ${ }^{68 a}$, I. L. Gavrilenko ${ }^{108}$, A. Gavrilyuk ${ }^{109}$, C. Gay ${ }^{172}$, G. Gaycken ${ }^{24}$, E. N. Gazis ${ }^{10}$, C. N. P. Gee ${ }^{141}$, J. Geisen ${ }^{51}$, M. Geisen ${ }^{97}$, M. P. Geisler ${ }^{59 a}$, K. Gellerstedt ${ }^{43 a}, 43 b$, C. Gemme ${ }^{53 b}$, M. H. Genest ${ }^{56}$, C. Geng $^{103}$, S. Gentile ${ }^{70 a}, 70 \mathrm{~b}$, S. George ${ }^{91}$, D. Gerbaudo ${ }^{14}$, G. Gessner ${ }^{45}$, S. Ghasemi ${ }^{148}$, M. Ghasemi Bostanabad ${ }^{173}$, M. Ghneimat ${ }^{24}$, B. Giacobbe ${ }^{23 b}$, S. Giagu ${ }^{70 a, 70 b}$, N. Giangiacomi ${ }^{23 a, 23 b}$, P. Giannetti ${ }^{69 a}$, A. Giannini ${ }^{67 a, 67 b}$, S. M. Gibson ${ }^{91}$, M. Gignac ${ }^{143}$, D. Gillberg ${ }^{33}$, G. Gilles ${ }^{179}$, D. M. Gingrich ${ }^{3 \text {,as }}$, M. P. Giordani64a,64c, F. M. Giorgi ${ }^{23 b}$, P. F. Giraud ${ }^{142}$, P. Giromini ${ }^{57}$, G. Giugliarelli ${ }^{64 a, 64 c}$, D. Giugni ${ }^{66 a}$, F. Giuli ${ }^{131}$, M. Giulini ${ }^{59 b}$, S. Gkaitatzis ${ }^{159}$, I. Gkialas ${ }^{9, i}$, E. L. Gkougkousis ${ }^{14}$, P. Gkountoumis ${ }^{10}$, L. K. Gladilin ${ }^{11}$, C. Glasman ${ }^{96}$, J. Glatzer ${ }^{14}$, P. C. F. Glaysher ${ }^{44}$, A. Glazov ${ }^{44}$, M. Goblirsch-Kolb ${ }^{26}$, J. Godlewski ${ }^{82}$, S. Goldfarb ${ }^{102}$, T. Golling ${ }^{52}$, D. Golubkov ${ }^{140}, \quad$ A. Gomes ${ }^{136 a, 136 b, 136 d,}$ R. Goncalves Gama ${ }^{78 a}$, R. Gonçalo ${ }^{136 a}$, G. Gonella ${ }^{50}$, L. Gonella ${ }^{21}$, A. Gongadze ${ }^{77}$, F. Gonnella ${ }^{21}$ ，J. L. Gonski ${ }^{57}$ ， S. González de la Hoz ${ }^{171}$, S. Gonzalez-Sevilla ${ }^{52}$, L. Goossens ${ }^{35}$, P. A. Gorbounov ${ }^{109}$, H. A. Gordon ${ }^{29}$, B. Gorini ${ }^{35}$, E. Gorini ${ }^{65 a, 65 b}$, A. Gorišek ${ }^{89}$, A. T. Goshaw ${ }^{47}$, C. Gössling ${ }^{45}$, M. I. Gostkin ${ }^{77}$, C. A. Gottardo ${ }^{24}$, C. R. Goudet ${ }^{128}$, D. Goujdami ${ }^{34 c}$, A. G. Goussiou ${ }^{145}$, N. Govender ${ }^{32 b, b}$, C. Goy ${ }^{5}$, E. Gozani ${ }^{157}$, I. Grabowska-Bold ${ }^{81 a}$, P. O. J. Gradin ${ }^{169}$, E. C. Graham ${ }^{88}$, J. Gramling ${ }^{168}$, E. Gramstad ${ }^{130}$, S. Grancagnolo ${ }^{19}$ ， V. Gratchev ${ }^{134}$, P. M. Gravila ${ }^{27 f}$, F. G. Gravili65a,65b , C. Gray ${ }^{55}$, H. M. Gray ${ }^{18}$, Z. D. Greenwood ${ }^{93, \text { ai }}$,C. Grefe ${ }^{24}$, K. Gregersen ${ }^{92}$,I. M. Gregor ${ }^{44}$,P. Grenier ${ }^{150}$, K. Grevtsov ${ }^{44}$, J. Griffiths ${ }^{8}$, A. A. Grillo ${ }^{143}$, K. Grimm ${ }^{150}$, S. Grinstein ${ }^{14, y}$, Ph. Gris ${ }^{37}$, J.-F. Grivaz ${ }^{128}$, S. Groh ${ }^{97}$, E. Gross ${ }^{177}$, J. Grosse-Knetter ${ }^{51}$, G. C. Grossi ${ }^{93}$, Z. J. Grout ${ }^{92}$, C. Grud ${ }^{103}$, A. Grummer ${ }^{116}$, L. Guan ${ }^{103}$, W. Guan ${ }^{178}$, J. Guenther ${ }^{35}$, A. Guerguichon ${ }^{128}$, F. Guescini ${ }^{165 a}$, D. Guest ${ }^{168}$, R. Gugel ${ }^{50}$, B. Gui ${ }^{122}$, T. Guillemin' ${ }^{5}$, S. Guindon ${ }^{35}$, U. Gul ${ }^{55}$, C. Gumpert ${ }^{35}$, J. Guo ${ }^{58 c}$, W. Guo ${ }^{103}$, 
Y. Guo ${ }^{58 a, r}$ ，Z. Guo ${ }^{99}$ ，R. Gupta ${ }^{41}$ ，S. Gurbuz ${ }^{12 c}$ ，G. Gustavino ${ }^{124}$ ，B. J. Gutelman ${ }^{157 ， P . ~ G u t i e r r e z}{ }^{124}$ ，C. Gutschow ${ }^{92}$,

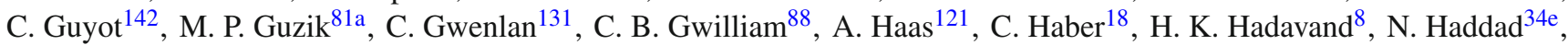
A. Hadef ${ }^{58 a}$, S. Hageböck ${ }^{24}$, M. Hagihara ${ }^{166}, \quad$ H. Hakobyan ${ }^{181, *}, \quad$ M. Haleem ${ }^{174}$, J. Haley ${ }^{125}$, G. Halladjian ${ }^{104}$, G. D. Hallewell ${ }^{19}$ ， K. Hamacher ${ }^{179}$ ， P. Hamal ${ }^{126}$ ， K. Hamano ${ }^{173}$ ， A. Hamilton ${ }^{32 a}$ ， G. N. Hamity ${ }^{146}$, K. Han ${ }^{58 a, a h}$, L. Han ${ }^{58 a}$, S. Han ${ }^{15 d}$, K. Hanagaki ${ }^{79, u}$, M. Hance ${ }^{143}$, D. M. Handl ${ }^{112}$, B. Haney ${ }^{133}$, R. Hankache ${ }^{132}$, P. Hanke ${ }^{59 a}$, E. Hansen ${ }^{94}$, J. B. Hansen ${ }^{39}$, J. D. Hansen ${ }^{39}$, M. C. Hansen ${ }^{24}$, P. H. Hansen ${ }^{39}$, K. Hara ${ }^{166}$, A. S. Hard ${ }^{178}$, T. Harenberg ${ }^{179}$, S. Harkusha ${ }^{105}$, P. F. Harrison ${ }^{175}$, N. M. Hartmann ${ }^{112}$, Y. Hasegawa ${ }^{147}$, A. Hasib ${ }^{48}$, S. Hassani ${ }^{142}$, S. Haug ${ }^{20}$, R. Hauser ${ }^{104}$,

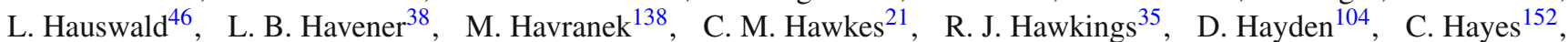
C. P. Hays ${ }^{131}$, J. M. Hays ${ }^{90}$, H. S. Hayward ${ }^{88}$, S. J. Haywood ${ }^{141}$, M. P. Heath ${ }^{48}$, V. Hedberg ${ }^{94}$, L. Heelan ${ }^{8}$, S. Heer ${ }^{24}$,

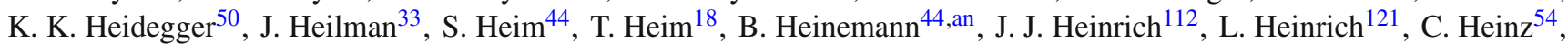
J. Hejbal ${ }^{137}$, L. Helary ${ }^{35}$, A. Held ${ }^{172}$, S. Hellesund ${ }^{130}$, S. Hellman ${ }^{43 a, 43 b}$, C. Helsens ${ }^{35}$, R. C. W. Henderson ${ }^{87}$, Y. Heng ${ }^{178}$, S. Henkelmann ${ }^{172}$ ， A. M. Henriques Correia ${ }^{35}$ ， G. H. Herbert ${ }^{19}$ ， H. Herde ${ }^{26}$, V. Herget ${ }^{174}$, Y. Hernández Jiménez ${ }^{32 c}$, H. Herr ${ }^{97}$ ， M. G. Herrmann ${ }^{112}$ ， G. Herten ${ }^{50}$ ， R. Hertenberger ${ }^{112}$ ， L. Hervas ${ }^{35}$ ， T. C. Herwig ${ }^{133}$ ， G. G. Hesketh ${ }^{92}$, N. P. Hessey ${ }^{165 a}$ ， J. W. Hetherly ${ }^{41}$ ， S. Higashino ${ }^{79}$, E. Higón-Rodriguez ${ }^{171}$ ，K. Hildebrand ${ }^{36}$, E. Hill ${ }^{173}$, J. C. Hill ${ }^{31}$, K. K. Hill ${ }^{29}$ ， K. H. Hiller ${ }^{44}$ ， S. J. Hillier ${ }^{21}$ ， M. Hils ${ }^{46}$ ， I. Hinchliffe ${ }^{18}$ ，M. Hirose ${ }^{129}$ ，D. Hirschbuehl ${ }^{179}$ ， B. Hiti ${ }^{89}$, O. Hladik $^{137}$, D. R. Hlaluku ${ }^{32 \mathrm{c}}, \quad$ X. Hoad ${ }^{48}$ J. Hobbs ${ }^{152}$, N. Hod ${ }^{165 a}, \quad$ M. C. Hodgkinson ${ }^{146}$, A. Hoecker ${ }^{35}$, M. R. Hoeferkamp ${ }^{116}$ ， F. Hoenig ${ }^{112}$ ， D. Hohn ${ }^{24}$ ， D. Hohov ${ }^{128}$ ， T. R. Holmes ${ }^{36}$, M. Holzbock ${ }^{112}$ ， M. Homann ${ }^{45}$, S. Honda ${ }^{166}$, T. Honda ${ }^{79}$, T. M. Hong ${ }^{135}$, A. Hönle ${ }^{113}$, B. H. Hooberman ${ }^{170}$, W. H. Hopkins ${ }^{127}$, Y. Horii ${ }^{115}$, P. Horn ${ }^{46}$, A. J. Horton ${ }^{149}$, L. A. Horyn ${ }^{36}$, J-Y. Hostachy ${ }^{56}$, A. Hostiuc ${ }^{145}$, S. Hou ${ }^{155}$, A. Hoummada ${ }^{34 a}$, J. Howarth ${ }^{98}$, J. Hoya ${ }^{86}$, M. Hrabovsky ${ }^{126}$, J. Hrdinka ${ }^{35}$, I. Hristova ${ }^{19}$, J. Hrivnac ${ }^{128}$, A. Hrynevich ${ }^{106}$, T. Hryn'ova ${ }^{5}$, P. J. Hsu ${ }^{62}$, S.-C. Hsu ${ }^{145}$, Q. $\mathrm{Hu}^{29}$ ， S. Hu ${ }^{58 c}$ ， Y. Huang ${ }^{15 a}$ ， Z. Hubacek ${ }^{138}$, F. Hubaut ${ }^{99}$, M. Huebner ${ }^{24}$, F. Huegging ${ }^{24}$ ， T. B. Huffman ${ }^{131}$,

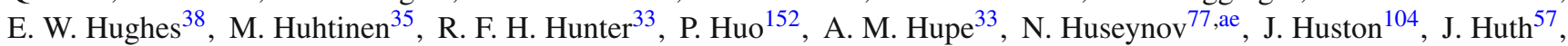
R. Hyneman ${ }^{103}$, G. Iacobucci ${ }^{52}$ ， G. Iakovidis ${ }^{29}$, I. Ibragimov ${ }^{148}$, L. Iconomidou-Fayard ${ }^{128}$, Z. Idrissi ${ }^{34 e}$, P. Iengo ${ }^{35}$,

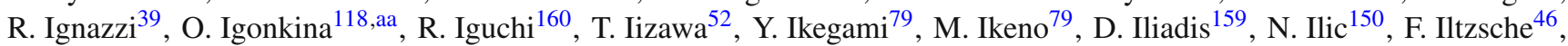
G. Introzzi ${ }^{68 a}, 68 b, \quad M$. Iodice $^{72 a}$, K. Iordanidou ${ }^{38}$, V. Ippolito ${ }^{70 a, 70 b}, \quad$ M. F. Isacson ${ }^{169}$ ， N. Ishijima ${ }^{129}$, M. Ishino ${ }^{160}$, M. Ishitsuka ${ }^{162}$, W. Islam ${ }^{125}$, C. Issever ${ }^{131}$, S. Istin ${ }^{12 c, a m}$, F. Ito ${ }^{166}$, J. M. Iturbe Ponce ${ }^{61 a}$, R. Iuppa ${ }^{73 a, 73 b}$, A. Ivina ${ }^{177}$, H. Iwasaki ${ }^{79}$, J. M. Izen ${ }^{42}$, V. Izzo ${ }^{67 a}$, P. Jacka ${ }^{137}$, P. Jackson ${ }^{1}$, R. M. Jacobs ${ }^{24}$, V. Jain ${ }^{2}$, G. Jäkel ${ }^{179}$, K. B. Jakobi ${ }^{97}$, K. Jakobs ${ }^{50}$ ， S. Jakobsen ${ }^{74}$, T. Jakoubek ${ }^{137}$, D. O. Jamin ${ }^{125}$ ， D. K. Jana ${ }^{93}$ ， R. Jansky ${ }^{52}$ ， J. Janssen ${ }^{24}$, M. Janus ${ }^{51}$, P. A. Janus ${ }^{81 a}$, G. Jarlskog ${ }^{94}$, N. Javadov ${ }^{77, a e}$, T. Javưrek ${ }^{50}$, M. Javurkova ${ }^{50}$, F. Jeanneau ${ }^{142}$, L. Jeanty ${ }^{18}$, J. Jejelava ${ }^{156 a, a f}$, A. Jelinskas ${ }^{175}$, P. Jenni ${ }^{50, \text { c }}$, J. Jeong ${ }^{44}$, S. Jézéquel ${ }^{5}$, H. Ji ${ }^{178}$, J. Jia ${ }^{152}$, H. Jiang ${ }^{76}$, Y. Jiang ${ }^{58 a}$, Z. Jiang ${ }^{150, p}$, S. Jiggins ${ }^{50}$, F. A. Jimenez Morales ${ }^{37}$ ， J. Jimenez Pena ${ }^{171}$ ， S. Jin ${ }^{15 c}$ ， A. Jinaru ${ }^{27 b}$ ， O. Jinnouchi ${ }^{162}$, H. Jivan ${ }^{32 c}$ ， P. Johansson ${ }^{146}$, K. A. Johns ${ }^{7}$, C. A. Johnson ${ }^{63}$, W. J. Johnson ${ }^{145}$, K. Jon-And ${ }^{43 a, 43 b}$, R. W. L. Jones ${ }^{87}$, S. D. Jones ${ }^{153}$, S. Jones ${ }^{7}$, T. J. Jones ${ }^{88}$ ， J. Jongmanns ${ }^{59 a}$ ，P. M. Jorge ${ }^{136 a, 136 b}$ ，J. Jovicevic ${ }^{165 a}$ ， X. Ju ${ }^{178}$ ，J. J. Junggeburth ${ }^{113}$ ，A. Juste Rozas ${ }^{14, y}$, A. Kaczmarska ${ }^{82}$, M. Kado ${ }^{128}$, H. Kagan ${ }^{122}$, M. Kagan ${ }^{150}$, T. Kaji ${ }^{176}$, E. Kajomovitz ${ }^{157}$, C. W. Kalderon ${ }^{94}$, A. Kaluza ${ }^{97}$, S. Kama ${ }^{41}$, A. Kamenshchikov ${ }^{140}$, L. Kanjir ${ }^{89}$, Y. Kano ${ }^{160}$, V. A. Kantserov ${ }^{110}$, J. Kanzaki ${ }^{79}$, B. Kaplan ${ }^{121}$, L. S. Kaplan ${ }^{178}$, D. $\operatorname{Kar}^{32 \mathrm{c}}$, M. J. Kareem ${ }^{165 b}$, E. Karentzos ${ }^{10}$, S. N. Karpov ${ }^{77}$, Z. M. Karpova ${ }^{77}$, V. Kartvelishvili ${ }^{87}$, A. N. Karyukhin ${ }^{140}$, K. Kasahara ${ }^{166}$, L. Kashif ${ }^{178}$, R. D. Kass ${ }^{122}$, A. Kastanas ${ }^{151}$, Y. Kataoka ${ }^{160}$, C. Kato ${ }^{160}$ ， J. Katzy ${ }^{44}$, K. Kawade ${ }^{80}$, K. Kawagoe ${ }^{85}$, T. Kawamoto ${ }^{160}$ ， G. Kawamura ${ }^{51}$, E. F. Kay ${ }^{88}$, V. F. Kazaninina,120b， R. Keeler ${ }^{173}$, R. Kehoe ${ }^{41}$,

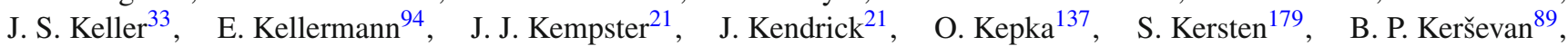
R. A. Keyes ${ }^{101}$, M. Khader ${ }^{170}$, F. Khalil-Zada ${ }^{13}$, A. Khanov ${ }^{125}$, A. G. Kharlamov ${ }^{120 a, 120 b}$, T. Kharlamova ${ }^{120 a, 120 b}$, A. Khodinov ${ }^{163}$, T. J. Khoo ${ }^{52}$, E. Khramov ${ }^{77}$, J. Khubua ${ }^{156 b}$, S. Kido ${ }^{80}$, M. Kiehn ${ }^{52}$, C. R. Kilby ${ }^{91}$, S. H. Kim ${ }^{166}$, Y. K. $\mathrm{Kim}^{36}$, N. Kimura ${ }^{64 a, 64 c}$, O. M. Kind ${ }^{19}$, B. T. King ${ }^{88}$, D. Kirchmeier ${ }^{46}$, J. Kirk ${ }^{141}$, A. E. Kiryunin ${ }^{113}$, T. Kishimoto ${ }^{160}$, D. Kisielewska ${ }^{81 a}$, V. Kitali ${ }^{44}$, O. Kivernyk ${ }^{5}$, E. Kladiva ${ }^{28 b}$, T. Klapdor-Kleingrothaus ${ }^{50}$, M. H. Klein ${ }^{103}$, M. Klein ${ }^{88}$, U. Klein ${ }^{88}$, K. Kleinknecht ${ }^{97}$, P. Klimek ${ }^{119}$, A. Klimentov ${ }^{29}$, R. Klingenberg ${ }^{45,}$, T. Klingl ${ }^{24}$, T. Klioutchnikova ${ }^{35}$,

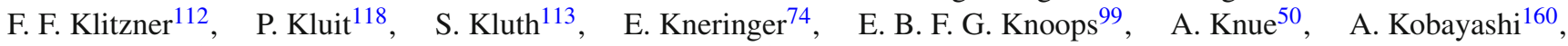
D. Kobayashi ${ }^{85}$, T. Kobayashi ${ }^{160}$, M. Kobel ${ }^{46}$, M. Kocian ${ }^{150}$, P. Kodys ${ }^{139}$, T. Koffas ${ }^{33}$, E. Koffeman ${ }^{118}$, N. M. Köhler ${ }^{113}$, T. Koi ${ }^{150}$, M. Kolb ${ }^{59 b}$ ， I. Koletsou ${ }^{5}$, T. Kondo ${ }^{79}$, N. Kondrashova ${ }^{58 c}$, K. Köneke ${ }^{50}$, A. C. König ${ }^{117}, \quad$ T. Kono ${ }^{79}$, R. Konoplich ${ }^{121, \text { aj }}$, V. Konstantinides ${ }^{92}$, N. Konstantinidis ${ }^{92}$, B. Konya ${ }^{94}$, R. Kopeliansky ${ }^{63}$, S. Koperny ${ }^{81 a}$, K. Korcyl ${ }^{82}$, K. Kordas ${ }^{159}$, A. Korn ${ }^{92}$, I. Korolkov ${ }^{14}$, E. V. Korolkova ${ }^{146}$, O. Kortner ${ }^{113}$ ， S. Kortner ${ }^{113}$, T. Kosek ${ }^{139}$, V. V. Kostyukhin ${ }^{24}$, A. Kotwal ${ }^{47}$, A. Koulouris ${ }^{10}$, A. Kourkoumeli-Charalampidi ${ }^{68 a}$,68b , C. Kourkoumelis ${ }^{9}$, E. Kourlitis ${ }^{146}$,

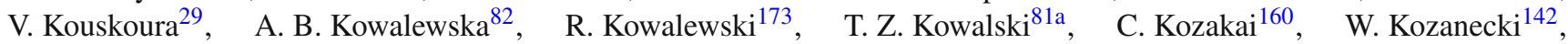
A. S. Kozhin ${ }^{140}$, V. A. Kramarenko ${ }^{111}$, G. Kramberger ${ }^{89}$, D. Krasnopevtsev ${ }^{110}$, M. W. Krasny ${ }^{132}$, A. Krasznahorkay ${ }^{35}$, D. Krauss ${ }^{113}$, J. A. Kremer ${ }^{81 a}$, J. Kretzschmar ${ }^{88}$, P. Krieger ${ }^{164}$, K. Krizka ${ }^{18}$, K. Kroeninger ${ }^{45}$, H. Kroha ${ }^{113}$, J. Kroll ${ }^{137}$, 
J. Kroll ${ }^{133}$ ， J. Krstic ${ }^{16}$ ， U. Kruchonak ${ }^{77}$ ， H. Krüger ${ }^{24}$ ， N. Krumnack ${ }^{76}$ ， M. C. Kruse ${ }^{47}$ ， T. Kubota ${ }^{102}$ ， S. Kuday ${ }^{4 b}$, J. T. Kuechler ${ }^{179}$ ， S. Kuehn ${ }^{35}$ ， A. Kugel ${ }^{59 a}$ ， F. Kuger ${ }^{174}$, T. Kuhl ${ }^{44}$ ， V. Kukhtin ${ }^{77}$ ， R. Kukla ${ }^{99}$, Y. Kulchitsky ${ }^{105}$, S. Kuleshov ${ }^{144 b}$, Y. P. Kulinich ${ }^{170}$, M. Kuna ${ }^{56}$, T. Kunigo ${ }^{83}$, A. Kupco ${ }^{137}$, T. Kupfer ${ }^{45}$, O. Kuprash ${ }^{158}$, H. Kurashige ${ }^{80}$, L. L. Kurchaninov ${ }^{165 a}$, Y. A. Kurochkin ${ }^{105}$, M. G. Kurth ${ }^{15 d}$, E. S. Kuwertz ${ }^{173}$, M. Kuze ${ }^{162}$ ， J. Kvita ${ }^{126}$, T. Kwan ${ }^{101}$, A. La Rosa ${ }^{113}$ ， J. L. La Rosa Navarro ${ }^{78 d}$ ， L. La Rotonda ${ }^{40 a, 40 b}$ ， F. La Ruffa ${ }^{40 a, 40 b}$ ， C. Lacasta ${ }^{171}$, F. Lacava ${ }^{70 a, 70 b}$,

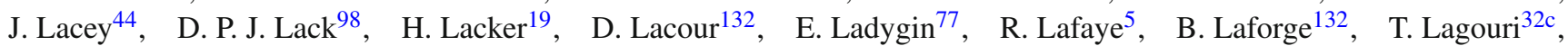
S. Lai ${ }^{51}$, S. Lammers ${ }^{63}$, W. Lampl ${ }^{7}$, E. Lançon ${ }^{29}$, U. Landgraf ${ }^{50}$, M. P. J. Landon ${ }^{90}$, M. C. Lanfermann ${ }^{52}$, V. S. Lang ${ }^{44}$, J. C. Lange ${ }^{14}$, R. J. Langenberg ${ }^{35}$, A. J. Lankford ${ }^{168}$, F. Lanni ${ }^{29}$, K. Lantzsch $^{24}$, A. Lanza ${ }^{68 a}$, A. Lapertosa ${ }^{53 a, 53 b}$, S. Laplace ${ }^{132}$, J. F. Laporte ${ }^{142}$, T. Lari ${ }^{66 a}$, F. Lasagni Manghi ${ }^{23 a}$,23b,$\quad$ M. Lassnig ${ }^{35}$, T. S. Lau ${ }^{61 a}$, A. Laudrain ${ }^{128}$, M. Lavorgna ${ }^{67 a, 67 b}$, A. T. Law ${ }^{143}$, P. Laycock ${ }^{88}, \quad$ M. Lazzaroni ${ }^{66 a, 66 b}, \quad$ B. Le ${ }^{102}$, O. Le Dortz ${ }^{132}$, E. Le Guirriec ${ }^{99}$,

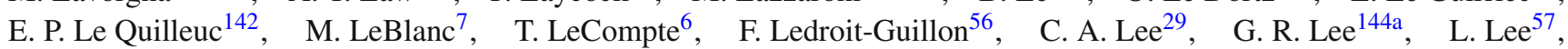
S. C. Lee ${ }^{155}$, B. Lefebvre ${ }^{101}$, M. Lefebvre ${ }^{173}$, F. Legger ${ }^{112}$, C. Leggett ${ }^{18}$, N. Lehmann ${ }^{179}$ ， G. Lehmann Miotto ${ }^{35}$, W. A. Leight ${ }^{44}$, A. Leisos ${ }^{159, v}$, M. A. L. Leite ${ }^{78 d}$, R. Leitner ${ }^{139}$, D. Lellouch ${ }^{177}$, B. Lemmer ${ }^{51}$, K. J. C. Leney ${ }^{92}$, T. Lenz ${ }^{24}$, B. Lenzi ${ }^{35}$, R. Leone ${ }^{7}$, S. Leone ${ }^{69 a}$, C. Leonidopoulos ${ }^{48}$, G. Lerner ${ }^{153}$, C. Leroy ${ }^{107}$, R. Les ${ }^{164}$, A. A. J. Lesage ${ }^{142}$, C. G. Lester $^{31}$ ， M. Levchenko ${ }^{134}$ ， J. Levêque ${ }^{5}$, D. Levin ${ }^{103}$, L. J. Levinson ${ }^{177}$ ， D. Lewis ${ }^{90}$ ， B. Li ${ }^{103}$, C-Q. Li ${ }^{58 a}$,

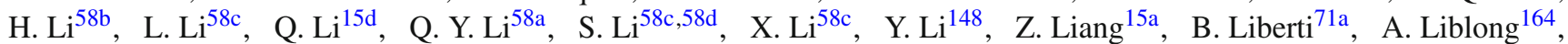

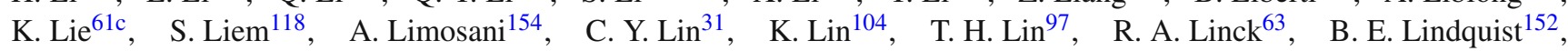
A. L. Lionti ${ }^{52}$, E. Lipeles ${ }^{133}, \quad$ A. Lipniacka ${ }^{17}, \quad$ M. Lisovyi ${ }^{59 b}, \quad$ T. M. Liss ${ }^{170, \text { ap }, \quad \text { A. Lister }}{ }^{172}, \quad$ A. M. Litke ${ }^{143}$,

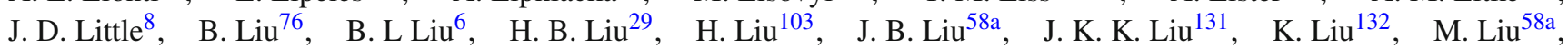

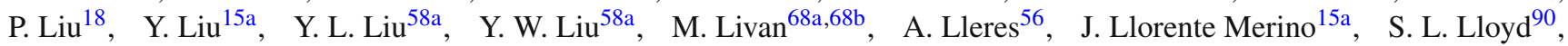
C. Y. Lo ${ }^{61 b}$, F. Lo Sterzo ${ }^{41}$, E. M. Lobodzinska ${ }^{44}$, P. Loch ${ }^{7}$, A. Loesle ${ }^{50}$, K. M. Loew ${ }^{26}$, T. Lohse ${ }^{19}$, K. Lohwasser ${ }^{146}$, M. Lokajicek ${ }^{137}$, B. A. Long ${ }^{25}$, J. D. Long ${ }^{170}$, R. E. Long ${ }^{87}$, L. Longo ${ }^{65 a, 65 b}$, K. A. Looper ${ }^{122}$, J. A. Lopez ${ }^{144 b}$,

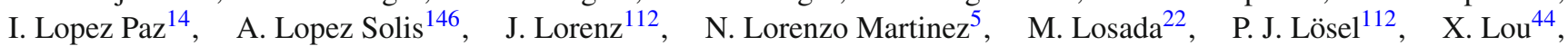

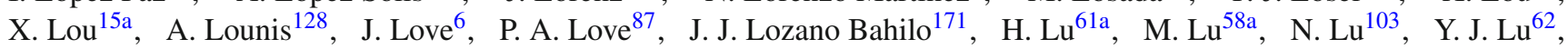
H. J. Lubatti ${ }^{145}$, C. Luci $^{70 a}$,70b , A. Lucotte ${ }^{56}$, C. Luedtke ${ }^{50}$, F. Luehring ${ }^{63}$, I. Luise ${ }^{132}$, W. Lukas ${ }^{74}$, L. Luminari ${ }^{70 a}$, B. Lund-Jensen ${ }^{151}$, M. S. Lutz ${ }^{100}$, P. M. Luzi ${ }^{132}$, D. Lynn ${ }^{29}$, R. Lysak ${ }^{137}$, E. Lytken ${ }^{94}$, F. Lyu ${ }^{15 a}$, V. Lyubushkin ${ }^{77}$, H. Ma ${ }^{29}$, L. L. Ma ${ }^{58 b}$, Y. Ma ${ }^{58 b}$, G. Maccarrone ${ }^{49}$, A. Macchiolo ${ }^{113}$, C. M. Macdonald ${ }^{146}$, J. Machado Miguens ${ }^{133,136 b}$, D. Madaffari ${ }^{171}$, R. Madar ${ }^{37}$, W. F. Mader ${ }^{46}$, A. Madsen ${ }^{44}$, N. Madysa ${ }^{46}$, J. Maeda ${ }^{80}$, K. Maekawa ${ }^{160}$, S. Maeland ${ }^{17}$, T. Maeno ${ }^{29}$, A. S. Maevskiy ${ }^{111}$, V. Magerl ${ }^{50}$, C. Maidantchik ${ }^{78 b}$, T. Maier ${ }^{112}$, A. Maio ${ }^{136 a, 136 b, 136 d}$, O. Majersky ${ }^{28 a}$, S. Majewski ${ }^{127}$, Y. Makida ${ }^{79}$, N. Makovec ${ }^{128}$, B. Malaescu ${ }^{132}$, Pa. Malecki ${ }^{82}$, V. P. Maleev ${ }^{134}$, F. Malek ${ }^{56}$, U. Mallik ${ }^{75}$, D. Malon ${ }^{6}$, C. Malone ${ }^{31}$, S. Maltezos ${ }^{10}$, S. Malyukov ${ }^{35}$, J. Mamuzic ${ }^{171}$, G. Mancini ${ }^{49}$, I. Mandićc9, J. Maneira ${ }^{136 a}$, L. Manhaes de Andrade Filho ${ }^{78 a}$, J. Manjarres Ramos ${ }^{46}$, K. H. Mankinen ${ }^{94}$, A. Mann ${ }^{112}$, A. Manousos ${ }^{74}$, B. Mansoulie ${ }^{142}$, J. D. Mansour ${ }^{15 a}$, M. Mantoani ${ }^{51}$, S. Manzoni ${ }^{66 a, 66 b}$, G. Marceca ${ }^{30}$, L. March ${ }^{52}$, L. Marchese ${ }^{131}$, G. Marchiori ${ }^{132}$,

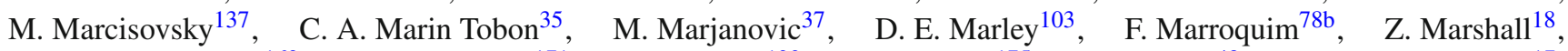
M. U. F Martensson ${ }^{169}$, S. Marti-Garcia ${ }^{171}$, C. B. Martin ${ }^{122}$, T. A. Martin ${ }^{175}$, V. J. Martin ${ }^{48}$, B. Martin dit Latour ${ }^{17}$, M. Martinez ${ }^{14, y}$, V. I. Martinez Outschoorn ${ }^{100}$, S. Martin-Haugh ${ }^{141}$, V. S. Martoiu ${ }^{27 b}$, A. C. Martyniuk ${ }^{92}$, A. Marzin ${ }^{35}$, L. Masetti ${ }^{97}$, T. Mashimo ${ }^{160}$, R. Mashinistov ${ }^{108}$, J. Masik ${ }^{98}$, A. L. Maslennikov ${ }^{120 a, 120 b}$, L. H. Mason ${ }^{102}$, L. Massa ${ }^{71 a, 71 b}$, P. Massarotti 67a,67b, P. Mastrandrea ${ }^{5}$, A. Mastroberardino ${ }^{40 a, 40 b}$, T. Masubuchi ${ }^{160}$, P. Mättig ${ }^{179}$, J. Maurer ${ }^{27 b}$, B. Maček ${ }^{89}$, S. J. Maxfield ${ }^{88}$, D. A. Maximov ${ }^{120 a, 120 b}$, R. Mazini ${ }^{155}$, I. Maznas ${ }^{159}$, S. M. Mazza ${ }^{143}$, N. C. Mc Fadden ${ }^{116}$, G. Mc Goldrick ${ }^{164}$, S. P. Mc Kee ${ }^{103}$, A. McCarn ${ }^{103}$, T. G. McCarthy ${ }^{113}$, L. I. McClymont ${ }^{92}$, E. F. McDonald ${ }^{102}$,

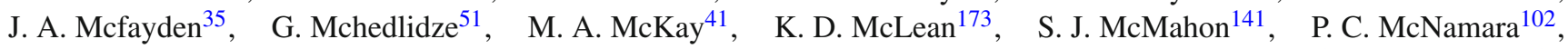

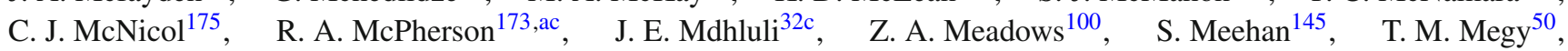
S. Mehlhase ${ }^{112}$, A. Mehta ${ }^{88}$, T. Meideck ${ }^{56}$, B. Meirose ${ }^{42}$, D. Melini ${ }^{171, g}$, B. R. Mellado Garcia ${ }^{32 c}$, J. D. Mellenthin ${ }^{51}$, M. Melo ${ }^{28 a}$, F. Meloni44, A. Melzer ${ }^{24}$, S. B. Menary ${ }^{98}$, E. D. Mendes Gouveia ${ }^{136 a}$, L. Meng ${ }^{88,}$ X. T. Meng ${ }^{103}$, A. Mengarellii ${ }^{23 a}, 23 b$, S. Menke ${ }^{113}$, E. Meoni ${ }^{40 a, 40 b}$, S. Mergelmeyer ${ }^{19}$, C. Merlassino ${ }^{20}$, P. Mermod ${ }^{52}$, L. Merola ${ }^{67 a, 67 b}$, C. Meroni6 ${ }^{66 a}$, F. S. Merritt ${ }^{36}$, A. Messina ${ }^{70 a}$,70b, J. Metcalfe $^{6}$, A. S. Mete ${ }^{168}$, C. Meyer ${ }^{133}$, J. Meyer ${ }^{157}$, J-P. Meyer ${ }^{142}$, H. Meyer Zu Theenhausen ${ }^{59 a}$, F. Miano ${ }^{153}$, R. P. Middleton ${ }^{141}$, L. Mijovićc ${ }^{48}$, G. Mikenberg ${ }^{177}$, M. Mikestikova ${ }^{137}$,

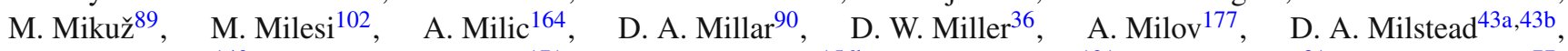
A. A. Minaenko ${ }^{140}$, M. Miñano Moya ${ }^{171}$, I. A. Minashvili ${ }^{156 b}, \quad$ A. I. Mincer ${ }^{121}$, B. Mindur ${ }^{81 a}$, M. Mineev ${ }^{77}$, Y. Minegishi ${ }^{160}$, Y. Ming ${ }^{178}$, L. M. Mir ${ }^{14}$, A. Mirto ${ }^{65 a, 65 b}$, K. P. Mistry ${ }^{133}$, T. Mitani ${ }^{176}$, J. Mitrevski ${ }^{112}$, V. A. Mitsou ${ }^{171}$, A. Miucci ${ }^{20}$, P. S. Miyagawa ${ }^{146}$, A. Mizukami ${ }^{79}$, J. U. Mjörnmark ${ }^{94}$, T. Mkrtchyan ${ }^{181}$, M. Mlynarikova ${ }^{139}$, T. Moa ${ }^{43 a, 43 b}$, K. Mochizuki ${ }^{107}$, P. Mogg 50 , S. Mohapatra ${ }^{38}$, S. Molander ${ }^{43 a, 43 b}$, R. Moles-Valls ${ }^{24}$, M. C. Mondragon ${ }^{104}$, K. Mönig ${ }^{44}$, J. Monk $^{39}$, E. Monnier ${ }^{99}$, A. Montalbano ${ }^{149}$, J. Montejo Berlingen ${ }^{35}$, F. Monticelli ${ }^{86}$, S. Monzani66a, R. W. Moore ${ }^{3}$, 
N. Morange ${ }^{128}$, D. Moreno ${ }^{22}$, M. Moreno Llácer ${ }^{35}$, P. Morettini ${ }^{53 b}$, M. Morgenstern ${ }^{118}$, S. Morgenstern ${ }^{46}$, D. Mori ${ }^{149}$, T. Mori ${ }^{160}$, M. Morii ${ }^{57}$, M. Morinaga ${ }^{176}$, V. Morisbak ${ }^{130}$, A. K. Morley ${ }^{35}$, G. Mornacchi ${ }^{35}$, A. P. Morris ${ }^{92}$, J. D. Morris ${ }^{90}$, L. Morvaj ${ }^{152}$, P. Moschovakos ${ }^{10}$, M. Mosidze ${ }^{156 b}, \quad$ H. J. Moss ${ }^{146}$, J. Moss ${ }^{150, m}$, K. Motohashi ${ }^{162}$, R. Mount ${ }^{150}$, E. Mountricha ${ }^{35}$, E. J. W. Moyse ${ }^{100}$, S. Muanza ${ }^{99}$, F. Mueller ${ }^{113}$, J. Mueller ${ }^{135}$, R. S. P. Mueller ${ }^{112}$, D. Muenstermann ${ }^{87}$, P. Mullen ${ }^{55}$, G. A. Mullier ${ }^{20}$, F. J. Munoz Sanchez ${ }^{98}$, P. Murin ${ }^{28 b}$, W. J. Murray ${ }^{141,175}$, A. Murrone ${ }^{66 a, 66 b}$, M. Muškinja ${ }^{89}$, C. Mwewa ${ }^{32 a}$, A. G. Myagkov ${ }^{140, a k}$, J. Myers ${ }^{127}$, M. Myska ${ }^{138}$, B. P. Nachman ${ }^{18}$, O. Nackenhorst ${ }^{45}, \quad$ K. Nagai ${ }^{131}$, K. Nagano ${ }^{79}$, Y. Nagasaka ${ }^{60}$, K. Nagata ${ }^{166}$, M. Nagel ${ }^{50}$, E. Nagy ${ }^{99}$, A. M. Nairz ${ }^{35}$, Y. Nakahama ${ }^{115}$, K. Nakamura ${ }^{79}$, T. Nakamura ${ }^{160}$, I. Nakano ${ }^{123}$, H. Nanjo ${ }^{129}$, F. Napolitano ${ }^{59 a}$, R. F. Naranjo Garcia ${ }^{44}$, R. Narayan ${ }^{11}$, D. I. Narrias Villar ${ }^{59 a}$, I. Naryshkin ${ }^{134}$, T. Naumann ${ }^{44}$, G. Navarro ${ }^{22}$, R. Nayyar ${ }^{7}$, H. A. Neal ${ }^{103}$, P. Y. Nechaeva ${ }^{108}$, T. J. Neep ${ }^{142}$, A. Negri6 ${ }^{68 a, 68 b}$,

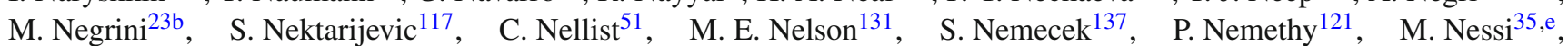
M. S. Neubauer ${ }^{170}$, M. Neumann ${ }^{179}$, P. R. Newman ${ }^{21}$, T. Y. Ng ${ }^{61 \mathrm{c}}$, Y. S. Ng ${ }^{19}$, H. D. N. Nguyen ${ }^{99}$, T. Nguyen Manh ${ }^{107}$, E. Nibigira ${ }^{37}$, R. B. Nickerson ${ }^{131}$, R. Nicolaidou ${ }^{142}$, J. Nielsen ${ }^{143}$, N. Nikiforou ${ }^{11}$, V. Nikolaenko ${ }^{140, \text { ak }}$, I. Nikolic-Audit ${ }^{132}$, K. Nikolopoulos ${ }^{21}$, P. Nilsson ${ }^{29}$, Y. Ninomiya ${ }^{79}$, A. Nisati ${ }^{70 a}$, N. Nishu ${ }^{58 c}$, R. Nisius ${ }^{113}$, I. Nitsche ${ }^{45}$, T. Nitta ${ }^{176}$, T. Nobe $^{160}$, Y. Noguchi $^{83}$, M. Nomachi ${ }^{129}$, I. Nomidis ${ }^{132}$, M. A. Nomura $^{29}$, T. Nooney ${ }^{90}$, M. Nordberg ${ }^{35}$, N. Norjoharuddeen ${ }^{131}$, T. Novak ${ }^{89}$, O. Novgorodova ${ }^{46}$, R. Novotny ${ }^{138}$, L. Nozka ${ }^{126}$, K. Ntekas ${ }^{168}$, E. Nurse ${ }^{92}$, F. Nuti ${ }^{102}$, F. G. Oakham ${ }^{33 \text {,as, }}$ H. Oberlack ${ }^{113}$, T. Obermann ${ }^{24}$, J. Ocariz ${ }^{132}$, A. Ochi ${ }^{80}$, I. Ochoa ${ }^{38}$, J. P. Ochoa-Ricoux ${ }^{144 a}$, K. O'Connor ${ }^{26}$, S. Oda ${ }^{85}$, S. Odaka ${ }^{79}$, S. Oerdek ${ }^{51}$, A. Oh ${ }^{98}$, S. H. Oh ${ }^{47}$, C. C. Ohm ${ }^{151}$, H. Oide ${ }^{53 a, 53 b}$, H. Okawa ${ }^{166}$, Y. Okazaki ${ }^{83}$, Y. Okumura ${ }^{160}$, T. Okuyama ${ }^{79}$, A. Olariu ${ }^{27 b}$, L. F. Oleiro Seabra ${ }^{136 a}$, S. A. Olivares Pino ${ }^{144 a}$, D. Oliveira Damazio ${ }^{29}$, J. L. Oliver ${ }^{1}$, M. J. R. Olsson ${ }^{36}$, A. Olszewski ${ }^{82}$, J. Olszowska ${ }^{82}$, D. C. O’Neil ${ }^{149}$, A. Onofre ${ }^{136 a, 136 e}$, K. Onogi ${ }^{115}$, P. U. E. Onyisi ${ }^{11}$, H. Oppen ${ }^{130}, \quad$ M. J. Oreglia ${ }^{36}$, Y. Oren ${ }^{158}, \quad$ D. Orestano ${ }^{72 a, 72 b}$, E. C. Orgill ${ }^{98}, \quad$ N. Orlando ${ }^{61 b}$, A. A. O'Rourke ${ }^{44}$, R. S. Orr ${ }^{164}$, B. Osculati ${ }^{53 a, 53 b, *}$, V. O'Shea ${ }^{55}$, R. Ospanov ${ }^{58 a}$, G. Otero y Garzon ${ }^{30}, \quad$ H. Otono ${ }^{85}$, M. Ouchrif ${ }^{34 d}$, F. Ould-Saada ${ }^{130}$, A. Ouraou ${ }^{142}$, Q. Ouyang ${ }^{15 a}$, M. Owen ${ }^{55}$, R. E. Owen ${ }^{21}$, V. E. Ozcan ${ }^{12 c}$, N. Ozturk ${ }^{8}$, J. Pacalt ${ }^{126}$, H. A. Pacey ${ }^{31}$, K. Pachal ${ }^{149}$, A. Pacheco Pages ${ }^{14}$, L. Pacheco Rodriguez ${ }^{142}$, C. Padilla Aranda ${ }^{14}$, S. Pagan Griso ${ }^{18}$, M. Paganini ${ }^{180}$, G. Palacino ${ }^{63}$, S. Palazzo ${ }^{40 a, 40 b}$, S. Palestini ${ }^{35}$, M. Palka ${ }^{81 b}$, D. Pallin ${ }^{37}$, I. Panagoulias ${ }^{10}$, C. E. Pandini ${ }^{35}$,

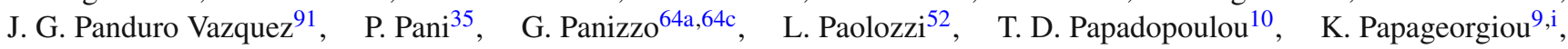
A. Paramonov6, D. Paredes Hernandez ${ }^{61 b}$, S. R. Paredes Saenz ${ }^{131}$, B. Parida ${ }^{58 c}$, A. J. Parker ${ }^{87}, \quad$ K. A. Parker ${ }^{44}$, M. A. Parker ${ }^{31}$, F. Parodi ${ }^{53 a, 53 b}$, J. A. Parsons ${ }^{38}$, U. Parzefall ${ }^{50}$, V. R. Pascuzzi ${ }^{164}$, J. M. P. Pasner ${ }^{143}$, E. Pasqualucci ${ }^{70 a}$, S. Passaggio ${ }^{53 b}$, F. Pastore ${ }^{91}$, P. Pasuwan ${ }^{43 a, 43 b}$, S. Pataraia ${ }^{97}$, J. R. Pater ${ }^{98}$, A. Pathak ${ }^{178, j}$, T. Pauly ${ }^{35}$, B. Pearson ${ }^{113}$, M. Pedersen ${ }^{130}$, L. Pedraza Diaz ${ }^{117}$, R. Pedro ${ }^{136 a, 136 b}$, S. V. Peleganchuk ${ }^{120 a, 120 b}$, O. Penc ${ }^{137}$, C. Peng ${ }^{15 d}$, H. Peng ${ }^{58 a}$, B. S. Peralva ${ }^{78 a}$, M. M. Perego ${ }^{142}$, A. P. Pereira Peixoto ${ }^{136 a}$, D. V. Perepelitsa ${ }^{29}$, F. Peri ${ }^{19}$, L. Perini ${ }^{66 a, 66 b}$, H. Pernegger ${ }^{35}$, S. Perrella ${ }^{67 a, 67 b}$, V. D. Peshekhonov ${ }^{77, *}$, K. Peters ${ }^{44}$, R. F. Y. Peters ${ }^{98}$, B. A. Petersen ${ }^{35}$, T. C. Petersen ${ }^{39}$, E. Petit ${ }^{56}$, A. Petridis ${ }^{1}$, C. Petridou ${ }^{159}$, P. Petroff ${ }^{128}$, E. Petrolo ${ }^{70 a}$, M. Petrov ${ }^{131}$, F. Petrucci ${ }^{72 a, 72 b}$, M. Pettee ${ }^{180}$, N. E. Pettersson ${ }^{100}$, A. Peyaud ${ }^{142}$, R. Pezoa ${ }^{144 b}$, T. Pham ${ }^{102}$, F. H. Phillips ${ }^{104}$, P. W. Phillips ${ }^{141}$, G. Piacquadio ${ }^{152}$, E. Pianori ${ }^{18}$, A. Picazio ${ }^{100}$, M. A. Pickering ${ }^{131}$, R. Piegaia ${ }^{30}$, J. E. Pilcher ${ }^{36}$, A. D. Pilkington ${ }^{98}$, M. Pinamonti ${ }^{71 a, 71 b}$, J. L. Pinfold ${ }^{3}$, M. Pitt ${ }^{177}$, M-A. Pleier ${ }^{29}$, V. Pleskot ${ }^{139}$, E. Plotnikova ${ }^{77}, \quad$ D. Pluth ${ }^{76}, \quad$ P. Podberezko ${ }^{120 a, 120 b}$, R. Poettgen ${ }^{94}, \quad$ R. Poggi ${ }^{52}$,

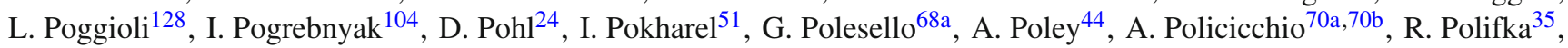
A. Polini2 ${ }^{23 b}$, C. S. Pollard ${ }^{44}$, V. Polychronakos ${ }^{29}$, D. Ponomarenko ${ }^{110}$, L. Pontecorvo ${ }^{70 a}$, G. A. Popeneciu ${ }^{27 d}$, D. M. Portillo Quintero ${ }^{132}$, S. Pospisil ${ }^{138}$, K. Potamianos $^{44}$, I. N. Potrap ${ }^{77}$, C. J. Potter ${ }^{31}$, H. Potti ${ }^{11}$, T. Poulsen ${ }^{94}$, J. Poveda ${ }^{35}$, T. D. Powell ${ }^{146}$, M. E. Pozo Astigarraga ${ }^{35}$, P. Pralavorio ${ }^{99}$, S. Prell ${ }^{76}$, D. Price ${ }^{98}$, M. Primavera ${ }^{65 a}$, S. Prince ${ }^{101}$, N. Proklova ${ }^{110}$, K. Prokofiev ${ }^{61 c}$, F. Prokoshin ${ }^{144 b}$, S. Protopopescu ${ }^{29}$, J. Proudfoot ${ }^{6}$, M. Przybycien ${ }^{81 a}$, A. Puri ${ }^{170}$, P. Puzo ${ }^{128}$, J. Qian ${ }^{103}$, Y. Qin ${ }^{98}$, A. Quadt ${ }^{51}$, M. Queitsch-Maitland ${ }^{44}$, A. Qureshi ${ }^{1}$, P. Rados ${ }^{102}$, F. Ragusa ${ }^{66 a, 66 b}$, G. Rahal ${ }^{95}$, J. A. Raine ${ }^{98}$, S. Rajagopalan ${ }^{29}$, A. Ramirez Morales ${ }^{90}$, T. Rashid ${ }^{128}$, S. Raspopov ${ }^{5}$, M. G. Ratti ${ }^{66 a, 66 b}$, D. M. Rauch ${ }^{44}$, F. Rauscher ${ }^{112}$, S. Rave ${ }^{97}$, B. Ravina ${ }^{146}$, I. Ravinovich ${ }^{177}$, J. H. Rawling ${ }^{98}, \quad$ M. Raymond ${ }^{35}$, A. L. Read $^{130}$, N. P. Readioff ${ }^{56}$, M. Reale ${ }^{65 a, 65 b}$, D. M. Rebuzzi ${ }^{68 a, 68 b}$, A. Redelbach ${ }^{174}$, G. Redlinger ${ }^{29}$, R. Reece ${ }^{143}$, R. G. Reed ${ }^{32 c}$, K. Reeves ${ }^{42}$, L. Rehnisch ${ }^{19}$, J. Reichert ${ }^{133}$, A. Reiss ${ }^{97}$, C. Rembser ${ }^{35}$, H. Ren ${ }^{15 \mathrm{~d}}$, M. Rescigno ${ }^{70 a}$, S. Resconi ${ }^{66 a}$, E. D. Resseguie ${ }^{133}$ ，S. Rettie ${ }^{172}$, E. Reynolds ${ }^{21}$, O. L. Rezanova ${ }^{120 a, 120 b}$, P. Reznicek ${ }^{139}$, R. Richter ${ }^{113}$, S. Richter ${ }^{92}$, E. Richter-Was ${ }^{81 b}$, O. Ricken ${ }^{24}$, M. Ridel $^{132}$, P. Rieck ${ }^{113}$, C. J. Riegel ${ }^{179}$, O. Rifki ${ }^{44}$, M. Rijssenbeek ${ }^{152}$, A. Rimoldi ${ }^{68 a, 68 b}$, M. Rimoldi ${ }^{20}$, L. Rinaldi ${ }^{23 b}$, G. Ripellino ${ }^{151}$, B. Ristic ${ }^{87}$, E. Ritsch ${ }^{35}$, I. Riu ${ }^{14}$, J. C. Rivera Vergara ${ }^{144 a}$,

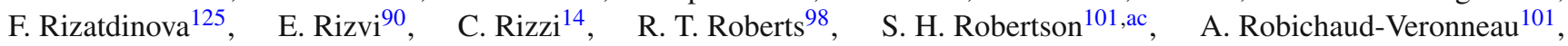
D. Robinson ${ }^{31}$, J. E. M. Robinson ${ }^{44}$, A. Robson ${ }^{55}$, E. Rocco ${ }^{97}$ ，C. Roda ${ }^{69 a}$,69b , Y. Rodina ${ }^{99}$ ， S. Rodriguez Bosca ${ }^{171}$, A. Rodriguez Perez ${ }^{14}$ ，D. Rodriguez Rodriguez ${ }^{171}$ ，A. M. Rodríguez Vera ${ }^{165 b}$ ，S. Roe ${ }^{35}$ ，C. S. Rogan ${ }^{57}$, O. Røhne ${ }^{130}$, R. Röhrig ${ }^{113}$ ， C. P. A. Roland ${ }^{63}$ ， J. Roloff ${ }^{57}$ ，A. Romaniouk ${ }^{110}$ ， M. Romano ${ }^{23 a, 23 b}$ ， N. Rompotis ${ }^{88}$, M. Ronzani ${ }^{121}$,

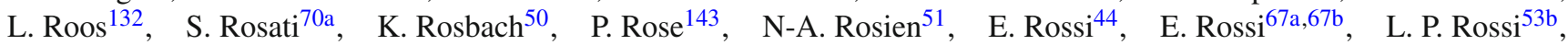


L. Rossini ${ }^{66 a, 66 b}$, J. H. N. Rosten ${ }^{31}$, R. Rosten ${ }^{14}$, M. Rotaru ${ }^{27 b}$, J. Rothberg ${ }^{145}$, D. Rousseau ${ }^{128}$, D. Roy ${ }^{32 c}$, A. Rozanov ${ }^{99}$,

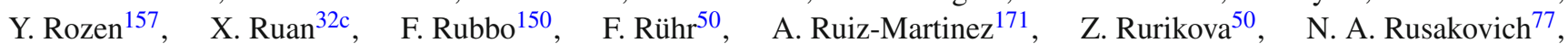
H. L. Russell ${ }^{101}$ ， J. P. Rutherfoord ${ }^{7}$ ， E. M. Rüttinger ${ }^{44, k}$ ，Y.F. Ryabov ${ }^{134}$ ， M. Rybar ${ }^{170 ， \quad \text { G. Rybkinn }}{ }^{128}$ ， S. Ryu ${ }^{6}$, A. Ryzhov ${ }^{140}$, G. F. Rzehorz ${ }^{51}$ ，P. Sabatini ${ }^{51}$ ， G. Sabato ${ }^{118}$ ， S. Sacerdoti ${ }^{128}$, H. F-W. Sadrozinski ${ }^{143}$, R. Sadykov ${ }^{77}$, F. Safai Tehrani ${ }^{70 a}$, P. Saha ${ }^{119}$, M. Sahinsoy ${ }^{59 a}$, A. Sahu ${ }^{179}$, M. Saimpert ${ }^{44}$, M. Saito ${ }^{160}$, T. Saito ${ }^{160}$, H. Sakamoto ${ }^{160}$, A. Sakharov ${ }^{121, a j}$ ，D. Salamani ${ }^{52}$ ，G. Salamanna ${ }^{72 a, 72 b}$ ， J. E. Salazar Loyola ${ }^{144 b}$ ，D. Salek ${ }^{118}$ ，P. H. Sales De Bruin ${ }^{169}$, D. Salihagic ${ }^{113}$, A. Salnikov ${ }^{150}$, J. Salt ${ }^{171}$, D. Salvatore ${ }^{40 a, 40 b}$, F. Salvatore ${ }^{153}$, A. Salvucci ${ }^{61 a, 61 b, 61 c}$, A. Salzburger ${ }^{35}$,

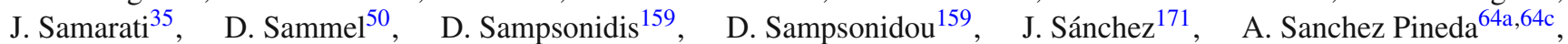
H. Sandaker ${ }^{130}$, C. O. Sander ${ }^{44}$, M. Sandhoff ${ }^{179}$, C. Sandoval ${ }^{22}$, D. P. C. Sankey ${ }^{141}$, M. Sannino ${ }^{53 a, 53 b}$, Y. Sano ${ }^{115}$, A. Sansoni ${ }^{49}$, C. Santoni ${ }^{37}$, H. Santos ${ }^{136 a}$, I. Santoyo Castillo ${ }^{153}$, A. Sapronov ${ }^{77}$, J. G. Saraiva ${ }^{136 a, 136 d}$, O. Sasaki ${ }^{79}$, K. Sato ${ }^{166}$, E. Sauvan ${ }^{5}$, P. Savard ${ }^{164, a s}$, N. Savic ${ }^{113}$, R. Sawada ${ }^{160}$, C. Sawyer ${ }^{141}$, L. Sawyer ${ }^{93, a i}$, C. Sbarra ${ }^{23 b}$, A. Sbrizzi ${ }^{23 a, 23 b}$, T. Scanlon ${ }^{92}$, J. Schaarschmidt ${ }^{145}$, P. Schacht ${ }^{113}$, B. M. Schachtner ${ }^{112}$, D. Schaefer ${ }^{36}$, L. Schaefer ${ }^{133}$, J. Schaeffer ${ }^{97}$ ，S. Schaepe ${ }^{35}$ ，U. Schäfer ${ }^{97}$ ，A. C. Schaffer ${ }^{128}$ ，D. Schaile ${ }^{112}$ ， R. D. Schamberger ${ }^{152}$, N. Scharmberg ${ }^{98}$, V. A. Schegelsky ${ }^{134}$, D. Scheirich ${ }^{139}$, F. Schenck ${ }^{19}$, M. Schernau ${ }^{168}$, C. Schiavi ${ }^{53 a, 53 b}$, S. Schier ${ }^{143}$, L. K. Schildgen ${ }^{24}$, Z. M. Schillaci ${ }^{26}$, E. J. Schioppa ${ }^{35}$, M. Schioppa ${ }^{40 a}, 40 b$, K. E. Schleicher ${ }^{50}$, S. Schlenker ${ }^{35}$, K. R. Schmidt-Sommerfeld ${ }^{113}$, K. Schmieden ${ }^{35}$, C. Schmitt ${ }^{97}$, S. Schmitt ${ }^{44}$, S. Schmitz ${ }^{97}$, U. Schnoor ${ }^{50}$, L. Schoeffel ${ }^{142}$, A. Schoening ${ }^{59 b}$, E. Schopf ${ }^{24}$, M. Schott ${ }^{97}$, J. F. P. Schouwenberg ${ }^{117}$, J. Schovancova ${ }^{35}$, S. Schramm ${ }^{52}$, A. Schulte ${ }^{97}, \quad$ H-C. Schultz-Coulon ${ }^{59 a}$, $\begin{array}{llll}\text { M. Schumacher } & \text { B } & \text { B. A. Schumm }\end{array}$ Ph. Schwemling ${ }^{142}$, R. Schwienhorst ${ }^{104}$, A. Sciandra ${ }^{24}$, G. Sciolla ${ }^{26}$, M. Scornajenghi ${ }^{40 a, 40 b}$, F. Scuri ${ }^{69 a}$, F. Scutti ${ }^{102}$,

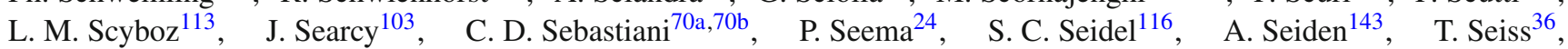
J. M. Seixas ${ }^{78 b}$, G. Sekhniaidze ${ }^{67 a}$, K. Sekhon ${ }^{103}$, S. J. Sekula ${ }^{41}$, N. Semprini-Cesari23a,23b, S. Sen ${ }^{47}$, S. Senkin ${ }^{37}$, C. Serfon ${ }^{130}$, L. Serin ${ }^{128}$, L. Serkin ${ }^{64 a, 64 b}$, M. Sessa ${ }^{72 a, 72 b}$, H. Severini ${ }^{124}$, F. Sforza ${ }^{167}$, A. Sfyrla ${ }^{52}$, E. Shabalina ${ }^{51}$, J. D. Shahinian ${ }^{143}$, N. W. Shaikh ${ }^{43 a, 43 b}$, L. Y. Shan ${ }^{15 a}$, R. Shang ${ }^{170}$, J. T. Shank ${ }^{25}$, M. Shapiro ${ }^{18}$, A. S. Sharma ${ }^{1}$, A. Sharma ${ }^{131}$, P. B. Shatalov ${ }^{109}$, K. Shaw ${ }^{153}$, S. M. Shaw ${ }^{98}$, A. Shcherbakova ${ }^{134}$, Y. Shen ${ }^{124}$, N. Sherafati ${ }^{33}$, A. D. Sherman ${ }^{25}$, P. Sherwood ${ }^{92}$, L. Shi ${ }^{155, \text { ao }}$, S. Shimizu ${ }^{80}$, C. O. Shimmin ${ }^{180}$, M. Shimojima ${ }^{114}$, I. P. J. Shipsey ${ }^{131}$, S. Shirabe ${ }^{85}$, M. Shiyakova ${ }^{77}$, J. Shlomi ${ }^{177}$, A. Shmeleva ${ }^{108}$, D. Shoaleh Saadi ${ }^{107}$, M. J. Shochet ${ }^{36}$, S. Shojaii ${ }^{102}$, D. R. Shope ${ }^{124}$, S. Shrestha ${ }^{122}$, E. Shulga ${ }^{110}$, P. Sicho ${ }^{137}$, A. M. Sickles ${ }^{170}$, P. E. Sidebo ${ }^{151}$, E. Sideras Haddad ${ }^{32 c}$, O. Sidiropoulou ${ }^{174}$, A. Sidoti23a,23b, F. Siegert ${ }^{46}$, Dj. Sijacki ${ }^{16}$, J. Silva ${ }^{136 a}$, M. Silva Jr. ${ }^{178}$, M. V. Silva Oliveira ${ }^{78 a}$, S. B. Silverstein ${ }^{43 a}$, L. Simic ${ }^{77}$, S. Simion ${ }^{128}$, E. Simioni ${ }^{97}$, M. Simon ${ }^{97}$, R. Simoniello ${ }^{97}$, P. Sinervo ${ }^{164}$, N. B. Sinev ${ }^{127}$, M. Sioli ${ }^{23 a, 23 b}$, G. Siragusa ${ }^{174}$, I. Siral ${ }^{103}$, S. Yu. Sivoklokov ${ }^{111}$, J. Sjölin ${ }^{43 a, 43 b}$, M. B. Skinner ${ }^{87}$, P. Skubic ${ }^{124}$, M. Slater ${ }^{21}$, T. Slavicek ${ }^{138}$, M. Slawinska ${ }^{82}$, K. Sliwa ${ }^{167}$, R. Slovak ${ }^{139}$, V. Smakhtin ${ }^{177}$, B. H. Smart5, J. Smiesko ${ }^{28 a}$, N. Smirnov ${ }^{110}$, S. Yu. Smirnov ${ }^{110}$, Y. Smirnov ${ }^{110}$, L. N. Smirnova ${ }^{111}$, O. Smirnova ${ }^{94}$, J. W. Smith ${ }^{51}$, M. N. K. Smith ${ }^{38}$, R. W. Smith ${ }^{38}$, M. Smizanska ${ }^{87}$, K. Smolek ${ }^{138}$, A. A. Snesarev ${ }^{108}$, I. M. Snyder ${ }^{127}$, S. Snyder ${ }^{29}$, R. Sobie ${ }^{173, a c}$, A. M. Soffa ${ }^{168}$, A. Soffer ${ }^{158}$, A. Søgaard ${ }^{48}$, D. A. Soh ${ }^{155}$, G. Sokhrannyi ${ }^{89}$, C. A. Solans Sanchez ${ }^{35}$, M. Solar ${ }^{138}$, E. Yu. Soldatov ${ }^{110}$, U. Soldevila ${ }^{171}$, A. A. Solodkov ${ }^{140}$, A. Soloshenko ${ }^{77}$, O. V. Solovyanov ${ }^{140}$, V. Solovyev ${ }^{134}$, P. Sommer ${ }^{146}$, H. Son ${ }^{167}$, W. Song ${ }^{141}$, A. Sopczak ${ }^{138}$, F. Sopkova ${ }^{28 b}$, D. Sosa ${ }^{59 b}$, C. L. Sotiropoulou ${ }^{69 a, 69 b}$, S. Sottocornola ${ }^{68 a, 68 b}$, R. Soualah ${ }^{64 a, 64 c, h}$,

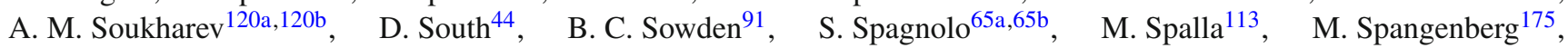
F. Spanò ${ }^{91}$, D. Sperlich ${ }^{19}$, F. Spettel ${ }^{113}$, T. M. Spieker ${ }^{59 a}$, R. Spighi ${ }^{23 b}$, G. Spigo ${ }^{35}$, L. A. Spiller ${ }^{102}$, D. P. Spiteri ${ }^{55}$,

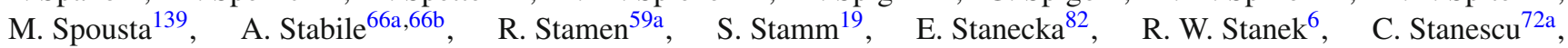
B. Stanislaus ${ }^{131}$, M. M. Stanitzki ${ }^{44}$, B. $\operatorname{Stapf}^{118}$, S. Stapnes ${ }^{130}$, E. A. Starchenko ${ }^{140}$, G. H. Stark ${ }^{36}$, J. Stark ${ }^{56}$, S. H Stark ${ }^{39}$, P. Staroba ${ }^{137}$, P. Starovoitov ${ }^{59 a}$, S. Stärz ${ }^{35}$, R. Staszewski ${ }^{82}$, M. Stegler ${ }^{44}$, P. Steinberg ${ }^{29}$, B. Stelzer ${ }^{149}$, H. J. Stelzer ${ }^{35}$, O. Stelzer-Chilton ${ }^{165 a}$, H. Stenzel ${ }^{54}$, T. J. Stevenson ${ }^{90}$, G. A. Stewart ${ }^{55}$, M. C. Stockton ${ }^{127}$, G. Stoicea ${ }^{27 b}$, P. Stolte ${ }^{51}$, S. Stonjek ${ }^{113}$, A. Straessner ${ }^{46}$ ，J. Strandberg ${ }^{151}$, S. Strandberg ${ }^{43 a, 43 b}$, M. Strauss ${ }^{124}$, P. Strizenec ${ }^{28 b}$, R. Ströhmer ${ }^{174}$, D. M. Strom ${ }^{127}$, R. Stroynowski ${ }^{41}$, A. Strubig ${ }^{48}$, S. A. Stucci ${ }^{29}$, B. Stugu ${ }^{17}$, J. Stupak ${ }^{124}$, N. A. Styles ${ }^{44}$, D. Su ${ }^{150}$, J. Su ${ }^{135}$, S. Suchek ${ }^{59 a}$, Y. Sugaya ${ }^{129}$, M. Suk ${ }^{138}$, V. V. Sulin ${ }^{108}$, D. M. S. Sultan ${ }^{52}$, S. Sultansoy ${ }^{4 c}$, T. Sumida ${ }^{83}$, S. Sun $^{103}$, X. Sun $^{3}$, K. Suruliz ${ }^{153}$, C. J. E. Suster ${ }^{154}$, M. R. Sutton ${ }^{153}$, S. Suzuki ${ }^{79}$, M. Svatos ${ }^{137}$, M. Swiatlowski ${ }^{36}$, S. P. Swift ${ }^{2}$, A. Sydorenko ${ }^{97}$, I. Sykora ${ }^{28 a}$, T. Sykora ${ }^{139}$, D. Ta ${ }^{97}$, K. Tackmann $^{44, z}$, J. Taenzer ${ }^{158}$, A. Taffard ${ }^{168}$, R. Tafirout ${ }^{165 a}$, E. Tahirovic ${ }^{90}$, N. Taiblum ${ }^{158}$, H. Takai ${ }^{29}$, R. Takashima ${ }^{84}$, E. H. Takasugi ${ }^{113}$, K. Takeda ${ }^{80}$, T. Takeshita ${ }^{147}$, Y. Takubo ${ }^{79}$, M. Talby ${ }^{99}$, A. A. Talyshev ${ }^{120 a, 120 b}$, J. Tanaka ${ }^{160}$, M. Tanaka ${ }^{162}$, R. Tanaka ${ }^{128}$, R. Tanioka ${ }^{80}$, B. B. Tannenwald ${ }^{122}$, S. Tapia Araya ${ }^{144 b}$, S. Tapprogge ${ }^{97}$, A. Tarek Abouelfadl Mohamed ${ }^{132}$, S. Tarem ${ }^{157}$, G. Tarna ${ }^{27 b, d}$, G. F. Tartarelli ${ }^{66 a}$, P. Tas ${ }^{139}, \quad$ M. Tasevsky ${ }^{137}$, T. Tashiro ${ }^{83}$, E. Tassi ${ }^{40 a, 40 b}$, A. Tavares Delgado ${ }^{136 a, 136 b}$, Y. Tayalati ${ }^{34 \mathrm{e}}$, A. C. Taylor ${ }^{116}$, A. J. Taylor ${ }^{48}$, G. N. Taylor ${ }^{102}$, P. T. E. Taylor ${ }^{102}$, W. Taylor ${ }^{165 b}$, A. S. Tee ${ }^{87}$, P. Teixeira-Dias ${ }^{91}, \quad$ H. Ten Kate ${ }^{35}$,

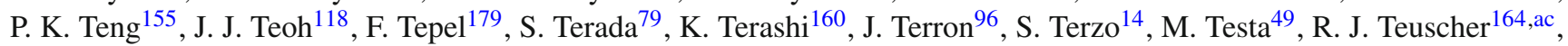


S. J. Thais ${ }^{180}, \quad$ T. Theveneaux-Pelzer ${ }^{44}, \quad$ F. Thiele ${ }^{39}, \quad$ J. P. Thomas ${ }^{21}, \quad$ A. S. Thompson ${ }^{55}, \quad$ P. D. Thompson ${ }^{21}$, L. A. Thomsen ${ }^{180}$, E. Thomson ${ }^{133}$, Y. $\operatorname{Tian}^{38}$, R. E. Ticse Torres $^{51}$, V. O. Tikhomirov ${ }^{108, a l}$, Yu. A. Tikhonov ${ }^{120 a, 120 b}$, S. Timoshenko ${ }^{110}$, P. Tipton ${ }^{180}$, S. Tisserant ${ }^{99}$, K. Todome ${ }^{162}$, S. Todorova-Nova ${ }^{5}$, S. Todt ${ }^{46}$, J. Tojo ${ }^{85}$, S. Tokár ${ }^{28 a}$, K. Tokushuku ${ }^{79}$, E. Tolley ${ }^{122}$, K. G. Tomiwa ${ }^{32 c}$, M. Tomoto ${ }^{115}$, L. Tompkins ${ }^{150, p}$, K. Toms ${ }^{116}$, B. Tong ${ }^{57}$, P. Tornambe ${ }^{50}$, E. Torrence ${ }^{127}$, H. Torres ${ }^{46}$, E. Torró Pastor ${ }^{145}$, C. Tosciri ${ }^{131}$, J. Toth ${ }^{99}$,ab , F. Touchard ${ }^{99}$, D. R. Tovey ${ }^{146}$, C. J. Treado ${ }^{121}$, T. Trefzger ${ }^{174}$, F. Tresoldi ${ }^{153}$, A. Tricoli ${ }^{29}$, I. M. Trigger ${ }^{165 a}$, S. Trincaz-Duvoid ${ }^{132}$, M. F. Tripiana ${ }^{14}$, W. Trischuk ${ }^{164}$,

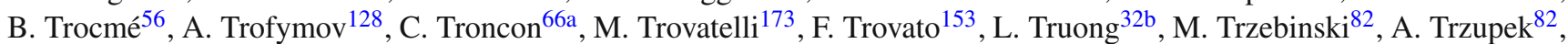
F. Tsai ${ }^{44}$, J.C-L. Tseng ${ }^{131}$, P. V. Tsiareshka ${ }^{105}$, N. Tsirintanis ${ }^{9}$, V. Tsiskaridze ${ }^{152}$, E. G. Tskhadadze ${ }^{156 a}$, I. I. Tsukerman ${ }^{109}$, V. Tsulaia ${ }^{18}$, S. Tsuno ${ }^{79}$, D. Tsybychev ${ }^{152}$, Y. Tu ${ }^{61 b}$, A. Tudorache ${ }^{27 b}$, V. Tudorache ${ }^{27 b}$, T. T. Tulbure ${ }^{27 a}$, A. N. Tuna ${ }^{57}$, S. Turchikhin ${ }^{77}$, D. Turgeman ${ }^{177}$, I. Turk Cakir ${ }^{4 b, t}$, R. Turra ${ }^{66 a}$, P. M. Tuts ${ }^{38}$, E. Tzovara ${ }^{97}$, G. Ucchielli ${ }^{23 a, 23 b}$, I. Ueda ${ }^{79}$, M. Ughetto ${ }^{43 a, 43 b}$, F. Ukegawa ${ }^{166}$, G. Unal ${ }^{35}$, A. Undrus ${ }^{29}$, G. Unel ${ }^{168}$, F. C. Ungaro ${ }^{102}$, Y. Unno ${ }^{79}$, K. Uno ${ }^{160}$, J. Urban ${ }^{28 b}$, P. Urquijo ${ }^{102}$, P. Urrejola ${ }^{97}$, G. Usai ${ }^{8}$, J. Usui ${ }^{79}$, L. Vacavant ${ }^{99}$, V. Vacek ${ }^{138}$, B. Vachon ${ }^{101}$, K. O. H. Vadla ${ }^{130}$,

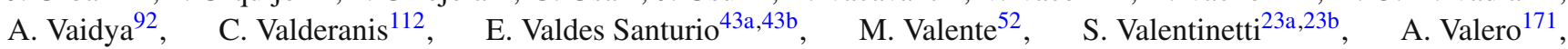
L. Valéry ${ }^{44}$, R. A. Vallance ${ }^{21}$, A. Vallier ${ }^{5}$, J. A. Valls Ferrer ${ }^{171}$, T. R. Van Daalen ${ }^{14}$, W. Van Den Wollenberg ${ }^{118}$, H. Van der Graaf ${ }^{118}$, P. Van Gemmeren ${ }^{6}$, J. Van Nieuwkoop ${ }^{149}$, I. Van Vulpen ${ }^{118}$, M. Vanadia ${ }^{71 a, 71 b}$, W. Vandelli ${ }^{35}$, A. Vaniachine ${ }^{163}$, P. Vankov ${ }^{118}$, R. Vari ${ }^{70}$, E. W. Varnes ${ }^{7}$, C. Varni ${ }^{53 a, 53 b}$, T. Varol ${ }^{41}$, D. Varouchas ${ }^{128}$, K. E. Varvell ${ }^{154}$, G. A. Vasquez ${ }^{144 \mathrm{~b}}$, J. G. Vasquez ${ }^{180}$, F. Vazeille ${ }^{37}$, D. Vazquez Furelos ${ }^{14}, \quad$ T. Vazquez Schroeder ${ }^{101}, \quad$ J. Veatch ${ }^{51}$, V. Vecchio ${ }^{72 a}$,72b, L. M. Veloce ${ }^{164}$, F. Veloso ${ }^{136 a, 136 c}$, S. Veneziano ${ }^{70 a}$, A. Ventura ${ }^{65 a, 65 b}$, M. Venturi ${ }^{173}$, N. Venturi ${ }^{35}$, V. Vercesi ${ }^{68 a}$, M. Verducci ${ }^{72 a, 72 b}$, C. M. Vergel Infante ${ }^{76}$, W. Verkerke ${ }^{118}$, A. T. Vermeulen ${ }^{118}$, J. C. Vermeulen ${ }^{118}$, M. C. Vetterli ${ }^{149}$,as, N. Viaux Maira ${ }^{144 b}$, M. Vicente Barreto Pinto ${ }^{52}$, I. Vichou ${ }^{170, *}$, T. Vickey ${ }^{146}$, O. E. Vickey Boeriu ${ }^{146}$, G. H. A. Viehhauser ${ }^{131}$, S. Viel ${ }^{18}$, L. Vigani ${ }^{131}$, M. Villa ${ }^{23 a, 23 b}$, M. Villaplana Perez ${ }^{66 a, 66 b}$, E. Vilucchi ${ }^{49}$, M. G. Vincter ${ }^{33}$, V. B. Vinogradov ${ }^{77}$, A. Vishwakarma ${ }^{44}$, C. Vittori ${ }^{23 a}, 23 b$, I. Vivarelli ${ }^{153}$, S. Vlachos ${ }^{10}$, M. Vogel ${ }^{179}$, P. Vokac ${ }^{138}$, G. Volpi $^{14}$, S. E. von Buddenbrock ${ }^{32 c}$, E. Von Toerne ${ }^{24}$, V. Vorobel ${ }^{139}$, K. Vorobev ${ }^{110}$, M. Vos ${ }^{171}$, J. H. Vossebeld ${ }^{88}$, N. Vranjes ${ }^{16}$, M. Vranjes Milosavljevic ${ }^{16}$, V. Vrba ${ }^{138}$, M. Vreeswijk ${ }^{118}$, T. Šfiligoj ${ }^{89}$, R. Vuillermet ${ }^{35}$, I. Vukotic ${ }^{36}, \quad$ T. Ženiš ${ }^{28 a}$, L. Živković ${ }^{16}$, P. Wagner ${ }^{24}$, W. Wagner ${ }^{179}$ ， J. Wagner-Kuhr ${ }^{112}$, H. Wahlberg ${ }^{86}$ ， S. Wahrmund ${ }^{46}$, K. Wakamiya ${ }^{80}$, V. M. Walbrecht ${ }^{113}$, J. Walder ${ }^{87}$, R. Walker ${ }^{112}$, S. D. Walker ${ }^{91}$, W. Walkowiak ${ }^{148}$, V. Wallangen ${ }^{43 a, 43 b}$, A. M. Wang ${ }^{57}$, C. Wang ${ }^{58 b, d}$, F. Wang ${ }^{178}$, H. Wang ${ }^{18}$, H. Wang ${ }^{3}$, J. Wang ${ }^{154}$, J. Wang ${ }^{59 b}$, P. Wang ${ }^{41}$, Q. Wang ${ }^{124}$, R.-J. Wang ${ }^{132}$, R. Wang ${ }^{58 a}$, R. Wang ${ }^{6}$, S. M. Wang ${ }^{155}$, W. T. Wang ${ }^{58 a}$, W. Wang ${ }^{15 c, a d}$, W. X. Wang ${ }^{58 a, a d}$, Y. Wang ${ }^{58 a}$, Z. Wang ${ }^{58 c}$, C. Wanotayaroj ${ }^{44}$, A. Warburton ${ }^{101}$, C. P. Ward ${ }^{31}$, D. R. Wardrope ${ }^{92}$, A. Washbrook ${ }^{48}$, P. M. Watkins ${ }^{21}$, A. T. Watson ${ }^{21}$, M. F. Watson ${ }^{21}$, G. Watts ${ }^{145}$, S. Watts ${ }^{98}$, B. M. Waugh ${ }^{92}$, A.F. Webb ${ }^{11}$, S. Webb ${ }^{97}$, C. Weber ${ }^{180}$, M. S. Weber ${ }^{20}$, S. A. Weber ${ }^{33}$, S. M. Weber ${ }^{59 a}$, A. R. Weidberg ${ }^{131}$, B. Weinert ${ }^{63}$, J. Weingarten ${ }^{51}$, M. Weirich ${ }^{97}$, C. Weiser ${ }^{50}$, P. S. Wells ${ }^{35}$, T. Wenaus ${ }^{29}$, T. Wengler ${ }^{35}$, S. Wenig ${ }^{35}$, N. Wermes ${ }^{24}$, M. D. Werner ${ }^{76}$, P. Werner ${ }^{35}$, M. Wessels ${ }^{59 a}$, T. D. Weston ${ }^{20}$, K. Whalen ${ }^{127}$, N. L. Whallon ${ }^{145}$, A. M. Wharton ${ }^{87}$, A. S. White ${ }^{103}$, A. White ${ }^{8}$, M. J. White ${ }^{1}$, R. White ${ }^{144 b}$, D. Whiteson ${ }^{168}$, B. W. Whitmore ${ }^{87}$, F. J. Wickens ${ }^{141}$, W. Wiedenmann ${ }^{178}$, M. Wielers ${ }^{141}$, C. Wiglesworth ${ }^{39}$, L. A. M. Wiik-Fuchs ${ }^{50}$, A. Wildauer ${ }^{113}$, F. Wilk ${ }^{98}$, H. G. Wilkens ${ }^{35}$, L. J. Wilkins ${ }^{91}$, H. H. Williams ${ }^{133}$, S. Williams ${ }^{31}$, C. Willis ${ }^{104}$, S. Willocq ${ }^{100}$, J. A. Wilson ${ }^{21}$, I. Wingerter-Seez ${ }^{5}$, E. Winkels ${ }^{153}$, F. Winklmeier ${ }^{127}$, O. J. Winston ${ }^{153}$, B. T. Winter ${ }^{24}$, M. Wittgen ${ }^{150}$,

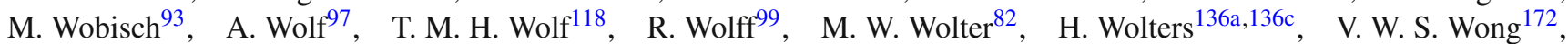
N. L. Woods ${ }^{143}$, S. D. Worm ${ }^{21}$, B. K. Wosiek ${ }^{82}$, K. W. Woźniak ${ }^{82}$ ，K. Wraight ${ }^{55}$ ，M. Wu ${ }^{36}$ ， S. L. Wu ${ }^{178}$ ，X. Wu ${ }^{52}$, Y. Wu ${ }^{58 a}$, T. R. Wyatt ${ }^{98}$, B. M. Wynne ${ }^{48}$, S. Xella ${ }^{39}$, Z. Xi ${ }^{103}$, L. Xia ${ }^{175}$, D. Xu ${ }^{15 a}$, H. Xu ${ }^{58 a}$, L. Xu ${ }^{29}$, T. Xu ${ }^{142}$, W. Xu ${ }^{103}$, B. Yabsley ${ }^{154}$, S. Yacoob ${ }^{32 a}$, K. Yajima ${ }^{129}$, D. P. Yallup ${ }^{92}$, D. Yamaguchi ${ }^{162}$, Y. Yamaguchi ${ }^{162}$, A. Yamamoto ${ }^{79}$, T. Yamanaka ${ }^{160}$, F. Yamane ${ }^{80}$, M. Yamatani ${ }^{160}$, T. Yamazaki ${ }^{160}$, Y. Yamazaki ${ }^{80}$, Z. Yan ${ }^{25}$, H. J. Yang ${ }^{58 c, 58 d}$, H. T. Yang ${ }^{18}$, S. Yang ${ }^{75}$, Y. Yang ${ }^{160}$, Z. Yang ${ }^{17}$, W-M. Yao ${ }^{18}$, Y. C. Yap ${ }^{44}$, Y. Yasu ${ }^{79}$, E. Yatsenko ${ }^{58 c, 58 d}$, J. Ye ${ }^{41}$, S. Ye ${ }^{29}$, I. Yeletskikh ${ }^{77}$, E. Yigitbasi ${ }^{25}$, E. Yildirim ${ }^{97}$, K. Yorita ${ }^{176}$, K. Yoshihara ${ }^{133}$, C. J. S. Young ${ }^{35}$, C. Young ${ }^{150}$, J. Yu ${ }^{8}$, J. Yu ${ }^{76}$, X. Yue ${ }^{59 a}$,

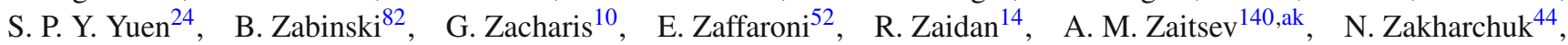
J. Zalieckas ${ }^{17}$, S. Zambito ${ }^{57}$, D. Zanzi ${ }^{35}$, D. R. Zaripovas ${ }^{55}$, S. V. Zeißner ${ }^{45}$, C. Zeitnitz ${ }^{179}$, G. Zemaityte ${ }^{131}$, J. C. Zeng ${ }^{170}$, Q. Zeng ${ }^{150}$, O. Zenin ${ }^{140}$, D. Zerwas ${ }^{128}$, M. Zgubič ${ }^{131}$, D. F. Zhang ${ }^{58 b}$, D. Zhang ${ }^{103}$, F. Zhang ${ }^{178}$, G. Zhang ${ }^{58 \mathrm{a}}$, H. Zhang ${ }^{15 \mathrm{c}}$, J. Zhang ${ }^{6}$, L. Zhang ${ }^{15 c}$, L. Zhang ${ }^{58 a}$, M. Zhang ${ }^{170}$, P. Zhang ${ }^{15 c}$, R. Zhang ${ }^{58 a}$, R. Zhang ${ }^{24}$, X. Zhang ${ }^{58 b}$, Y. Zhang ${ }^{15 d}$, Z. Zhang ${ }^{128}$, P. Zhao ${ }^{47}$, X. Zhao ${ }^{41}$, Y. Zhao ${ }^{58 b, 128, a h}$, Z. Zhao ${ }^{58 a}$, A. Zhemchugov ${ }^{77}$, B. Zhou ${ }^{103}$, C. Zhou ${ }^{178}$, L. Zhou ${ }^{41}$,

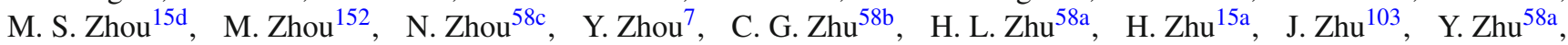
X. Zhuang ${ }^{15 a}$, K. Zhukov ${ }^{108}$, V. Zhulanov ${ }^{120 a, 120 b}$, A. Zibell ${ }^{174}$, D. Zieminska ${ }^{63}$, N. I. Zimine ${ }^{77}$, S. Zimmermann ${ }^{50}$, Z. Zinonos ${ }^{113}$, M. Zinser ${ }^{97}$, M. Ziolkowski ${ }^{148}$, G. Zobernig ${ }^{178}$, A. Zoccoli ${ }^{23 a, 23 b}$, K. Zoch ${ }^{51}$, T. G. Zorbas ${ }^{146}$, R. Zou ${ }^{36}$, M. Zur Nedden ${ }^{19}$, L. Zwalinski ${ }^{35}$ 
${ }^{1}$ Department of Physics, University of Adelaide, Adelaide, Australia

${ }^{2}$ Physics Department, SUNY Albany, Albany, NY, USA

${ }^{3}$ Department of Physics, University of Alberta, Edmonton, AB, Canada

4 (a) Department of Physics, Ankara University, Ankara, Turkey; ${ }^{(b)}$ Istanbul Aydin University, Istanbul, Turkey; ${ }^{(c)}$ Division of Physics, TOBB University of Economics and Technology, Ankara, Turkey

${ }^{5}$ LAPP, Université Grenoble Alpes, Université Savoie Mont Blanc, CNRS/IN2P3, Annecy, France

${ }^{6}$ High Energy Physics Division, Argonne National Laboratory, Argonne, IL, USA

${ }^{7}$ Department of Physics, University of Arizona, Tucson, AZ, USA

${ }^{8}$ Department of Physics, University of Texas at Arlington, Arlington, TX, USA

${ }^{9}$ Physics Department, National and Kapodistrian University of Athens, Athens, Greece

${ }^{10}$ Physics Department, National Technical University of Athens, Zografou, Greece

${ }^{11}$ Department of Physics, University of Texas at Austin, Austin, TX, USA

12 (a) Faculty of Engineering and Natural Sciences, Bahcesehir University, Istanbul, Turkey; ${ }^{(b)}$ Faculty of Engineering and Natural Sciences, Istanbul Bilgi University, Istanbul, Turkey; ${ }^{(c)}$ Department of Physics, Bogazici University, Istanbul, Turkey; ${ }^{(d)}$ Department of Physics Engineering, Gaziantep University, Gaziantep, Turkey

${ }^{13}$ Institute of Physics, Azerbaijan Academy of Sciences, Baku, Azerbaijan

${ }^{14}$ Institut de Física d'Altes Energies (IFAE), Barcelona Institute of Science and Technology, Barcelona, Spain

15 (a) Institute of High Energy Physics, Chinese Academy of Sciences, Beijing, China; ${ }^{(b)}$ Physics Department, Tsinghua University, Beijing, China; ${ }^{(c)}$ Department of Physics, Nanjing University, Nanjing, China; ${ }^{(d)}$ University of Chinese Academy of Science (UCAS), Beijing, China

${ }^{16}$ Institute of Physics, University of Belgrade, Belgrade, Serbia

${ }^{17}$ Department for Physics and Technology, University of Bergen, Bergen, Norway

${ }^{18}$ Physics Division, Lawrence Berkeley National Laboratory and University of California, Berkeley, CA, USA

${ }^{19}$ Institut für Physik, Humboldt Universität zu Berlin, Berlin, Germany

${ }^{20}$ Albert Einstein Center for Fundamental Physics and Laboratory for High Energy Physics, University of Bern, Bern, Switzerland

${ }^{21}$ School of Physics and Astronomy, University of Birmingham, Birmingham, UK

${ }^{22}$ Centro de Investigaciónes, Universidad Antonio Nariño, Bogotá, Colombia

23 (a) Dipartimento di Fisica e Astronomia, Università di Bologna, Bologna, Italy; ${ }^{(b)}$ INFN Sezione di Bologna, Bologna, Italy

${ }^{24}$ Physikalisches Institut, Universität Bonn, Bonn, Germany

${ }^{25}$ Department of Physics, Boston University, Boston, MA, USA

${ }^{26}$ Department of Physics, Brandeis University, Waltham, MA, USA

27 (a) Transilvania University of Brasov, Brasov, Romania; ${ }^{(b)}$ Horia Hulubei National Institute of Physics and Nuclear Engineering, Bucharest, Romania; ${ }^{(c)}$ Department of Physics, Alexandru Ioan Cuza University of Iasi, Iasi,

Romania; (d) Physics Department, National Institute for Research and Development of Isotopic and Molecular Technologies, Cluj-Napoca, Romania; ${ }^{\left({ }^{e}\right)}$ University Politehnica Bucharest, Bucharest, Romania; ${ }^{(f)}$ West University in Timisoara, Timisoara, Romania

28 (a) Faculty of Mathematics, Physics and Informatics, Comenius University, Bratislava, Slovakia; ${ }^{(b)}$ Department of Subnuclear Physics, Institute of Experimental Physics of the Slovak Academy of Sciences, Kosice, Slovak Republic

${ }^{29}$ Physics Department, Brookhaven National Laboratory, Upton, NY, USA

${ }^{30}$ Departamento de Física, Universidad de Buenos Aires, Buenos Aires, Argentina

${ }^{31}$ Cavendish Laboratory, University of Cambridge, Cambridge, UK

32 (a) Department of Physics, University of Cape Town, Cape Town, South Africa; ${ }^{(b)}$ Department of Mechanical Engineering Science, University of Johannesburg, Johannesburg, South Africa; ${ }^{(c)}$ School of Physics, University of the Witwatersrand, Johannesburg, South Africa

${ }^{33}$ Department of Physics, Carleton University, Ottawa, ON, Canada

34 (a) Faculté des Sciences Ain Chock, Réseau Universitaire de Physique des Hautes Energies-Université Hassan II, Casablanca, Morocco; ${ }^{(b)}$ Centre National de l'Energie des Sciences Techniques Nucleaires (CNESTEN), Rabat, Morocco; ${ }^{\left({ }^{c}\right)}$ Faculté des Sciences Semlalia, Université Cadi Ayyad, LPHEA-Marrakech, Marrakech, Morocco; ${ }^{(d)}$ Faculté des Sciences, Université Mohamed Premier and LPTPM, Oujda, Morocco; ${ }^{(e)}$ Faculté des sciences, Université Mohammed V, Rabat, Morocco

${ }^{35}$ CERN, Geneva, Switzerland 
${ }^{36}$ Enrico Fermi Institute, University of Chicago, Chicago, IL, USA

${ }^{37}$ LPC, Université Clermont Auvergne, CNRS/IN2P3, Clermont-Ferrand, France

${ }^{38}$ Nevis Laboratory, Columbia University, Irvington, NY, USA

${ }^{39}$ Niels Bohr Institute, University of Copenhagen, Copenhagen, Denmark

40 (a) Dipartimento di Fisica, Università della Calabria, Rende, Italy; ${ }^{(b)}$ INFN Gruppo Collegato di Cosenza, Laboratori

Nazionali di Frascati, Frascati, Italy

${ }^{41}$ Physics Department, Southern Methodist University, Dallas, TX, USA

42 Physics Department, University of Texas at Dallas, Richardson, TX, USA

43 (a) Department of Physics, Stockholm University, Stockholm, Sweden; ${ }^{(b)}$ Oskar Klein Centre, Stockholm, Sweden

${ }^{44}$ Deutsches Elektronen-Synchrotron DESY, Hamburg and Zeuthen, Germany

${ }^{45}$ Lehrstuhl für Experimentelle Physik IV, Technische Universität Dortmund, Dortmund, Germany

${ }^{46}$ Institut für Kern- und Teilchenphysik, Technische Universität Dresden, Dresden, Germany

${ }^{47}$ Department of Physics, Duke University, Durham, NC, USA

${ }^{48}$ SUPA-School of Physics and Astronomy, University of Edinburgh, Edinburgh, UK

${ }^{49}$ INFN e Laboratori Nazionali di Frascati, Frascati, Italy

${ }^{50}$ Physikalisches Institut, Albert-Ludwigs-Universität Freiburg, Freiburg, Germany

${ }^{51}$ II. Physikalisches Institut, Georg-August-Universität Göttingen, Göttingen, Germany

${ }^{52}$ Département de Physique Nucléaire et Corpusculaire, Université de Genève, Geneva, Switzerland

53 (a) Dipartimento di Fisica, Università di Genova, Genoa, Italy; ${ }^{(b)}$ INFN Sezione di Genova, Genoa, Italy

${ }^{54}$ II. Physikalisches Institut, Justus-Liebig-Universität Giessen, Giessen, Germany

55 SUPA-School of Physics and Astronomy, University of Glasgow, Glasgow, UK

${ }^{56}$ LPSC, Université Grenoble Alpes, CNRS/IN2P3, Grenoble INP, Grenoble, France

${ }^{57}$ Laboratory for Particle Physics and Cosmology, Harvard University, Cambridge, MA, USA

58 (a) Department of Modern Physics and State Key Laboratory of Particle Detection and Electronics, University of Science and Technology of China, Hefei, China; ${ }^{(b)}$ Institute of Frontier and Interdisciplinary Science and Key Laboratory of Particle Physics and Particle Irradiation (MOE), Shandong University, Qingdao, China; ${ }^{(c)}$ School of Physics and Astronomy, Shanghai Jiao Tong University, KLPPAC-MoE, SKLPPC, Shanghai, China; ${ }^{(d)}$ Tsung-Dao Lee Institute, Shanghai, China

59 (a) Kirchhoff-Institut für Physik, Ruprecht-Karls-Universität Heidelberg, Heidelberg, Germany; ${ }^{(b)}$ Physikalisches Institut, Ruprecht-Karls-Universität Heidelberg, Heidelberg, Germany

${ }^{60}$ Faculty of Applied Information Science, Hiroshima Institute of Technology, Hiroshima, Japan

61 (a) Department of Physics, Chinese University of Hong Kong, Shatin, NT, Hong Kong; ${ }^{\left({ }^{b}\right)}$ Department of Physics, University of Hong Kong, Hong Kong, China; ${ }^{(c)}$ Department of Physics and Institute for Advanced Study, Hong Kong University of Science and Technology, Clear Water Bay, Kowloon, Hong Kong, China

62 Department of Physics, National Tsing Hua University, Hsinchu, Taiwan

${ }^{63}$ Department of Physics, Indiana University, Bloomington, IN, USA

64 (a) INFN Gruppo Collegato di Udine, Sezione di Trieste, Udine, Italy; ${ }^{(b)}$ ICTP, Trieste, Italy; ${ }^{(c)}$ Dipartimento di Chimica, Fisica e Ambiente, Università di Udine, Udine, Italy

65 (a) INFN Sezione di Lecce, Lecce, Italy; ${ }^{(\mathrm{b})}$ Dipartimento di Matematica e Fisica, Università del Salento, Lecce, Italy

66 (a) INFN Sezione di Milano, Milan, Italy; ${ }^{(b)}$ Dipartimento di Fisica, Università di Milano, Milan, Italy

67 (a) INFN Sezione di Napoli, Naples, Italy; ${ }^{(b)}$ Dipartimento di Fisica, Università di Napoli, Naples, Italy

68 (a) INFN Sezione di Pavia, Pavia, Italy; ${ }^{(b)}$ Dipartimento di Fisica, Università di Pavia, Pavia, Italy

69 (a) INFN Sezione di Pisa, Pisa, Italy; ${ }^{(b)}$ Dipartimento di Fisica E. Fermi, Università di Pisa, Pisa, Italy

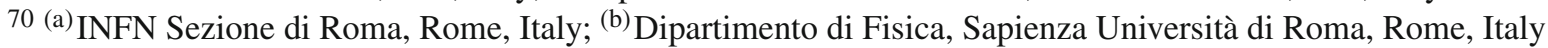

71 (a) INFN Sezione di Roma Tor Vergata, Rome, Italy; ${ }^{(b)}$ Dipartimento di Fisica, Università di Roma Tor Vergata, Rome, Italy

72 (a) INFN Sezione di Roma Tre, Rome, Italy; ${ }^{(b)}$ Dipartimento di Matematica e Fisica, Università Roma Tre, Rome, Italy

73 (a) INFN-TIFPA, Povo, Italy; ${ }^{(b)}$ Università degli Studi di Trento, Trento, Italy

${ }^{74}$ Institut für Astro- und Teilchenphysik, Leopold-Franzens-Universität, Innsbruck, Austria

75 University of Iowa, Iowa City, IA, USA

${ }^{76}$ Department of Physics and Astronomy, Iowa State University, Ames, IA, USA

77 Joint Institute for Nuclear Research, Dubna, Russia

78 (a) Departamento de Engenharia Elétrica, Universidade Federal de Juiz de Fora (UFJF), Juiz de Fora, 
Brazil; ${ }^{(b)}$ Universidade Federal do Rio De Janeiro COPPE/EE/IF, Rio de Janeiro, Brazil; ${ }^{(c)}$ Universidade Federal de São João del Rei (UFSJ), São João del Rei, Brazil; ${ }^{(d)}$ Instituto de Física, Universidade de São Paulo, São Paulo, Brazil

${ }^{79}$ KEK, High Energy Accelerator Research Organization, Tsukuba, Japan

${ }^{80}$ Graduate School of Science, Kobe University, Kobe, Japan

81 (a) Faculty of Physics and Applied Computer Science, AGH University of Science and Technology, Kraków,

Poland; ${ }^{(b)}$ Marian Smoluchowski Institute of Physics, Jagiellonian University, Kraków, Poland

${ }^{82}$ Institute of Nuclear Physics Polish Academy of Sciences, Kraków, Poland

${ }^{83}$ Faculty of Science, Kyoto University, Kyoto, Japan

${ }^{84}$ Kyoto University of Education, Kyoto, Japan

${ }^{85}$ Research Center for Advanced Particle Physics and Department of Physics, Kyushu University, Fukuoka, Japan

${ }^{86}$ Instituto de Física La Plata, Universidad Nacional de La Plata and CONICET, La Plata, Argentina

${ }^{87}$ Physics Department, Lancaster University, Lancaster, UK

${ }^{88}$ Oliver Lodge Laboratory, University of Liverpool, Liverpool, UK

${ }^{89}$ Department of Experimental Particle Physics, Jožef Stefan Institute and Department of Physics, University of Ljubljana, Ljubljana, Slovenia

${ }^{90}$ School of Physics and Astronomy, Queen Mary University of London, London, UK

${ }^{91}$ Department of Physics, Royal Holloway University of London, Egham, UK

92 Department of Physics and Astronomy, University College London, London, UK

${ }^{93}$ Louisiana Tech University, Ruston, LA, USA

${ }^{94}$ Fysiska institutionen, Lunds universitet, Lund, Sweden

${ }^{95}$ Centre de Calcul de l'Institut National de Physique Nucléaire et de Physique des Particules (IN2P3), Villeurbanne, France

${ }^{96}$ Departamento de Física Teorica C-15 and CIAFF, Universidad Autónoma de Madrid, Madrid, Spain

${ }^{97}$ Institut für Physik, Universität Mainz, Mainz, Germany

${ }^{98}$ School of Physics and Astronomy, University of Manchester, Manchester, UK

${ }^{99}$ CPPM, Aix-Marseille Université, CNRS/IN2P3, Marseille, France

100 Department of Physics, University of Massachusetts, Amherst, MA, USA

${ }^{101}$ Department of Physics, McGill University, Montreal, QC, Canada

102 School of Physics, University of Melbourne, Parkville, VIC, Australia

${ }^{103}$ Department of Physics, University of Michigan, Ann Arbor, MI, USA

${ }^{104}$ Department of Physics and Astronomy, Michigan State University, East Lansing, MI, USA

105 B.I. Stepanov Institute of Physics, National Academy of Sciences of Belarus, Minsk, Belarus

${ }^{106}$ Research Institute for Nuclear Problems of Byelorussian State University, Minsk, Belarus

${ }^{107}$ Group of Particle Physics, University of Montreal, Montreal, QC, Canada

${ }^{108}$ P.N. Lebedev Physical Institute of the Russian Academy of Sciences, Moscow, Russia

${ }^{109}$ Institute for Theoretical and Experimental Physics (ITEP), Moscow, Russia

110 National Research Nuclear University MEPhI, Moscow, Russia

${ }^{111}$ D.V. Skobeltsyn Institute of Nuclear Physics, M.V. Lomonosov Moscow State University, Moscow, Russia

${ }^{112}$ Fakultät für Physik, Ludwig-Maximilians-Universität München, Munich, Germany

${ }^{113}$ Max-Planck-Institut für Physik (Werner-Heisenberg-Institut), Munich, Germany

${ }^{114}$ Nagasaki Institute of Applied Science, Nagasaki, Japan

115 Graduate School of Science and Kobayashi-Maskawa Institute, Nagoya University, Nagoya, Japan

116 Department of Physics and Astronomy, University of New Mexico, Albuquerque, NM, USA

${ }^{117}$ Institute for Mathematics, Astrophysics and Particle Physics, Radboud University Nijmegen/Nikhef, Nijmegen, The Netherlands

${ }^{118}$ Nikhef National Institute for Subatomic Physics, University of Amsterdam, Amsterdam, The Netherlands

${ }^{119}$ Department of Physics, Northern Illinois University, De Kalb, IL, USA

120 (a) Budker Institute of Nuclear Physics, SB RAS, Novosibirsk, Russia; ${ }^{(b)}$ Novosibirsk State University, Novosibirsk, Russia

${ }^{121}$ Department of Physics, New York University, New York, NY, USA

122 Ohio State University, Columbus, OH, USA

${ }^{123}$ Faculty of Science, Okayama University, Okayama, Japan

${ }^{124}$ Homer L. Dodge Department of Physics and Astronomy, University of Oklahoma, Norman, OK, USA 
${ }^{125}$ Department of Physics, Oklahoma State University, Stillwater, OK, USA

${ }^{126}$ Palacký University, RCPTM, Joint Laboratory of Optics, Olomouc, Czech Republic

${ }^{127}$ Center for High Energy Physics, University of Oregon, Eugene, OR, USA

${ }^{128}$ LAL, Université Paris-Sud, CNRS/IN2P3, Université Paris-Saclay, Orsay, France

${ }^{129}$ Graduate School of Science, Osaka University, Osaka, Japan

${ }^{130}$ Department of Physics, University of Oslo, Oslo, Norway

${ }^{131}$ Department of Physics, Oxford University, Oxford, UK

${ }^{132}$ LPNHE, Sorbonne Université, Paris Diderot Sorbonne Paris Cité, CNRS/IN2P3, Paris, France

133 Department of Physics, University of Pennsylvania, Philadelphia, PA, USA

${ }^{134}$ Konstantinov Nuclear Physics Institute of National Research Centre "Kurchatov Institute", PNPI, St. Petersburg, Russia

135 Department of Physics and Astronomy, University of Pittsburgh, Pittsburgh, PA, USA

136 (a) Laboratório de Instrumentação e Física Experimental de Partículas-LIP, Lisbon, Portugal; ${ }^{(b)}$ Departamento de Física,

Faculdade de Ciências, Universidade de Lisboa, Lisbon, Portugal; ${ }^{(c)}$ Departamento de Física, Universidade de Coimbra,

Coimbra, Portugal; ${ }^{(\mathrm{d})}$ Centro de Física Nuclear da Universidade de Lisboa, Lisbon, Portugal; ${ }^{(\mathrm{e})}$ Departamento de Física, Universidade do Minho, Braga, Portugal; ${ }^{(f)}$ Departamento de Física Teorica y del Cosmos, Universidad de Granada,

Granada, Spain; ${ }^{(\mathrm{g})}$ Dep Física and CEFITEC of Faculdade de Ciências e Tecnologia, Universidade Nova de Lisboa,

Caparica, Portugal

${ }^{137}$ Institute of Physics, Academy of Sciences of the Czech Republic, Prague, Czech Republic

${ }^{138}$ Czech Technical University in Prague, Prague, Czech Republic

${ }^{139}$ Faculty of Mathematics and Physics, Charles University, Prague, Czech Republic

${ }^{140}$ State Research Center Institute for High Energy Physics, NRC KI, Protvino, Russia

${ }^{141}$ Particle Physics Department, Rutherford Appleton Laboratory, Didcot, UK

${ }^{142}$ IRFU, CEA, Université Paris-Saclay, Gif-sur-Yvette, France

${ }^{143}$ Santa Cruz Institute for Particle Physics, University of California Santa Cruz, Santa Cruz, CA, USA

144 (a) Departamento de Física, Pontificia Universidad Católica de Chile, Santiago, Chile; ${ }^{\left({ }^{b}\right)}$ Departamento de Física,

Universidad Técnica Federico Santa María, Valparaíso, Chile

145 Department of Physics, University of Washington, Seattle, WA, USA

${ }^{146}$ Department of Physics and Astronomy, University of Sheffield, Sheffield, UK

${ }^{147}$ Department of Physics, Shinshu University, Nagano, Japan

${ }^{148}$ Department Physik, Universität Siegen, Siegen, Germany

${ }^{149}$ Department of Physics, Simon Fraser University, Burnaby, BC, Canada

${ }^{150}$ SLAC National Accelerator Laboratory, Stanford, CA, USA

${ }^{151}$ Physics Department, Royal Institute of Technology, Stockholm, Sweden

152 Departments of Physics and Astronomy, Stony Brook University, Stony Brook, NY, USA

${ }^{153}$ Department of Physics and Astronomy, University of Sussex, Brighton, UK

${ }^{154}$ School of Physics, University of Sydney, Sydney, Australia

155 Institute of Physics, Academia Sinica, Taipei, Taiwan

156 (a) E. Andronikashvili Institute of Physics, Iv. Javakhishvili Tbilisi State University, Tbilisi, Georgia; ${ }^{(b)}$ High Energy

Physics Institute, Tbilisi State University, Tbilisi, Georgia

${ }^{157}$ Department of Physics, Technion, Israel Institute of Technology, Haifa, Israel

${ }^{158}$ Raymond and Beverly Sackler School of Physics and Astronomy, Tel Aviv University, Tel Aviv, Israel

${ }^{159}$ Department of Physics, Aristotle University of Thessaloniki, Thessaloniki, Greece

${ }^{160}$ International Center for Elementary Particle Physics and Department of Physics, University of Tokyo, Tokyo, Japan

${ }^{161}$ Graduate School of Science and Technology, Tokyo Metropolitan University, Tokyo, Japan

162 Department of Physics, Tokyo Institute of Technology, Tokyo, Japan

163 Tomsk State University, Tomsk, Russia

${ }^{164}$ Department of Physics, University of Toronto, Toronto, ON, Canada

165 (a) TRIUMF, Vancouver, BC, Canada; ${ }^{\left({ }^{b}\right)}$ Department of Physics and Astronomy, York University, Toronto, ON, Canada

166 Division of Physics and Tomonaga Center for the History of the Universe, Faculty of Pure and Applied Sciences,

University of Tsukuba, Tsukuba, Japan

${ }^{167}$ Department of Physics and Astronomy, Tufts University, Medford, MA, USA

168 Department of Physics and Astronomy, University of California Irvine, Irvine, CA, USA

${ }^{169}$ Department of Physics and Astronomy, University of Uppsala, Uppsala, Sweden 
${ }^{170}$ Department of Physics, University of Illinois, Urbana, IL, USA

${ }^{171}$ Instituto de Física Corpuscular (IFIC), Centro Mixto Universidad de Valencia - CSIC, Valencia, Spain

172 Department of Physics, University of British Columbia, Vancouver, BC, Canada

173 Department of Physics and Astronomy, University of Victoria, Victoria, BC, Canada

${ }^{174}$ Fakultät für Physik und Astronomie, Julius-Maximilians-Universität Würzburg, Würzburg, Germany

175 Department of Physics, University of Warwick, Coventry, UK

${ }^{176}$ Waseda University, Tokyo, Japan

177 Department of Particle Physics, Weizmann Institute of Science, Rehovot, Israel

${ }^{178}$ Department of Physics, University of Wisconsin, Madison, WI, USA

${ }^{179}$ Fakultät für Mathematik und Naturwissenschaften, Fachgruppe Physik, Bergische Universität Wuppertal, Wuppertal, Germany

${ }^{180}$ Department of Physics, Yale University, New Haven, CT, USA

181 Yerevan Physics Institute, Yerevan, Armenia

${ }^{\text {a }}$ Also at Borough of Manhattan Community College, City University of New York, NY, USA

${ }^{b}$ Also at Centre for High Performance Computing, CSIR Campus, Rosebank, Cape Town, South Africa

${ }^{c}$ Also at CERN, Geneva, Switzerland

d Also at CPPM, Aix-Marseille Université, CNRS/IN2P3, Marseille, France

${ }^{e}$ Also at Département de Physique Nucléaire et Corpusculaire, Université de Genève, Genève, Switzerland

${ }^{\mathrm{f}}$ Also at Departament de Fisica de la Universitat Autonoma de Barcelona, Barcelona, Spain

g Also at Departamento de Física Teorica y del Cosmos, Universidad de Granada, Granada (Spain), Spain

${ }^{\mathrm{h}}$ Also at Department of Applied Physics and Astronomy, University of Sharjah, Sharjah, United Arab Emirates

${ }^{i}$ Also at Department of Financial and Management Engineering, University of the Aegean, Chios, Greece

j Also at Department of Physics and Astronomy, University of Louisville, Louisville, KY, USA

${ }^{\mathrm{k}}$ Also at Department of Physics and Astronomy, University of Sheffield, Sheffield, UK

${ }^{1}$ Also at Department of Physics, California State University, Fresno, CA, USA

${ }^{m}$ Also at Department of Physics, California State University, Sacramento, CA, USA

${ }^{n}$ Also at Department of Physics, King's College London, London, UK

${ }^{o}$ Also at Department of Physics, St. Petersburg State Polytechnical University, St. Petersburg, Russia

p Also at Department of Physics, Stanford University, USA

q Also at Department of Physics, University of Fribourg, Fribourg, Switzerland

${ }^{r}$ Also at Department of Physics, University of Michigan, Ann Arbor, MI, USA

${ }^{\mathrm{s}}$ Also at Dipartimento di Fisica E. Fermi, Università di Pisa, Pisa, Italy

${ }^{t}$ Also at Giresun University, Faculty of Engineering, Giresun, Turkey

u Also at Graduate School of Science, Osaka University, Osaka, Japan

${ }^{v}$ Also at Hellenic Open University, Patras, Greece

${ }^{w}$ Also at Horia Hulubei National Institute of Physics and Nuclear Engineering, Bucharest, Romania

${ }^{x}$ Also at II Physikalisches Institut, Georg-August-Universität Göttingen, Göttingen, Germany

${ }^{y}$ Also at Institucio Catalana de Recerca i Estudis Avancats, ICREA, Barcelona, Spain

${ }^{\mathrm{z}}$ Also at Institut für Experimentalphysik, Universität Hamburg, Hamburg, Germany

${ }^{\text {aa }}$ Also at Institute for Mathematics, Astrophysics and Particle Physics, Radboud University Nijmegen/Nikhef, Nijmegen, The Netherlands

${ }^{a b}$ Also at Institute for Particle and Nuclear Physics, Wigner Research Centre for Physics, Budapest, Hungary

${ }^{\text {ac }}$ Also at Institute of Particle Physics (IPP), Canada

${ }^{\text {ad }}$ Also at Institute of Physics, Academia Sinica, Taipei, Taiwan

ae Also at Institute of Physics, Azerbaijan Academy of Sciences, Baku, Azerbaijan

af Also at Institute of Theoretical Physics, Ilia State University, Tbilisi, Georgia

ag Also at Istanbul University, Department of Physics, Istanbul, Turkey

ah Also at LAL, Université Paris-Sud, CNRS/IN2P3, Université Paris-Saclay, Orsay, France

ai Also at Louisiana Tech University, Ruston, LA, USA

aj Also at Manhattan College, New York, NY, USA

ak Also at Moscow Institute of Physics and Technology State University, Dolgoprudny, Russia

al Also at National Research Nuclear University MEPhI, Moscow, Russia 
am Also at Near East University, Nicosia, North Cyprus, Mersin, Turkey

${ }^{a n}$ Also at Physikalisches Institut, Albert-Ludwigs-Universität Freiburg, Freiburg, Germany

ao Also at School of Physics, Sun Yat-sen University, Guangzhou, China

ap Also at The City College of New York, New York, NY, USA

aq Also at The Collaborative Innovation Center of Quantum Matter (CICQM), Beijing, China

${ }^{\text {ar }}$ Also at Tomsk State University, Tomsk, and Moscow Institute of Physics and Technology State University, Dolgoprudny, Russia

as Also at TRIUMF, Vancouver, BC, Canada

${ }^{\text {at }}$ Also at Universita di Napoli Parthenope, Naples, Italy

* Deceased 